\title{
Targeting cyclic nucleotide phosphodiesterase 5 (PDE5) in brain: Toward the development of a PET radioligand labeled with fluorine-18
}

Barbara Wenzel ${ }^{1 \S \#, ~ J i a n r o n g ~ L i u ² \#, ~ S l a d j a n a ~ D u k i c-S t e f a n o v i c ~}{ }^{1}$, Winnie Deuther-Conrad ${ }^{1}$, Rodrigo Teodoro ${ }^{1}$, Friedrich-Alexander Ludwig $^{1}$, Jean-Michel Chezal ${ }^{2}$, Emmanuel Moreau², Peter Brust ${ }^{1}$, Aurelie Maisonial-Besset ${ }^{2}$

${ }^{1}$ Helmholtz-Zentrum Dresden-Rossendorf, Institute of Radiopharmaceutical Cancer Research, Department of Neuroradiopharmaceuticals, Leipzig, Germany;

2 UMR 1240 INSERM IMOST, Université Clermont-Auvergne, Clermont-Ferrand, France

\# These authors contributed equally to the work.

$\S$ corresponding author

\begin{abstract}
With the aim to develop a specific radioligand for imaging the cyclic nucleotide phosphodiesterase 5 (PDE5) in brain by positron emission tomography (PET), seven new fluorinated inhibitors $(3-9)$ were synthesized on the basis of a quinoline core. The inhibitory activity for PDE5 together with a panel of other PDEs was determined in vitro and two derivatives were selected for $\mathrm{IC}_{50}$ value determination. The most promising compound $\mathbf{7}\left(\mathrm{IC}_{50}\right.$ $=5.92 \mathrm{nM}$ for PDE5A), containing a 3-fluoroazetidine moiety, was further radiolabeled by aliphatic nucleophilic substitution of two different leaving groups (nosylate and tosylate) using $\left[{ }^{18} \mathrm{~F}\right]$ fluoride. The use of the nosylate precursor and tetra- $n$-butyl ammonium $\left[{ }^{18} \mathrm{~F}\right]$ fluoride $\left(\left[{ }^{18} \mathrm{~F}\right]\right.$ TBAF $)$ in 3-methyl-3-pentanol combined with the addition of a small amount of water proved to be the best radiolabeling conditions achieving a RCY of $4.9 \pm 1.5 \%$ in an automated procedure. Preliminary biological investigations in vitro and in vivo were performed to characterize this new PDE5 radioligand. Metabolism studies of $\left[{ }^{18} \mathrm{~F}\right] 7$ in mice revealed a fast metabolic degradation with the formation of radiometabolites which have been detected in the brain.
\end{abstract}

Keywords: PDE5, ${ }^{18} \mathrm{~F}$-radiolabeling, nosylate, tosylate, ${ }^{18} \mathrm{~F}$-fluoroazetidine 


\section{Introduction}

The cyclic nucleotide phosphodiesterases (PDEs) are a large family of enzymes that regulate intracellular levels of the two important second messengers cyclic adenosine $3^{\prime}, 5^{\prime}$ monophosphate (cAMP) and cyclic guanosine $3^{\prime}, 5^{\prime}$-monophosphate (cGMP) by cleavage of their phosphodiester bond. To date, 11 subtypes of these enzymes have been identified and classified mainly according to their amino acid sequences. Phosphodiesterase 5 (PDE5) is specific for cGMP which is generated by the nitric oxide (NO)-dependent soluble guanylate cyclase and activates the protein kinase G (PKG). PKG activation triggers phosphorylation of numerous intracellular proteins that in turn regulate many primary physiological functions such as modulation of vascular tone and vasorelaxation in vascular smooth muscle. This was the basis for the breakthrough of one of the most celebrated PDE5 inhibitors namely sildenafil $\left(\right.$ Viagra $\left.^{\circledR}\right)$ which is used as treatment for erectile dysfunction and chronic pulmonary hypertension. Moreover, currently this enzyme arouses increasing interest of clinical researchers as a multifunctional biomarker and drug target because PDE5 inhibition also affects neurodegenerative [1] and cancerous processes [2]. For example, increased PDE5 levels have been reported in several carcinomas including colon adenocarcinoma, bladder squamous carcinoma, thyroid tumors, metastatic breast, prostate, pancreatic, bladder and lung cancers [3-9]. As it was demonstrated, that cGMP can reduce cell growth and induce apoptosis $[10,11]$, and that PDE5 catalyzes the degradation of cGMP, this enzyme has been suggested to be involved in tumor progression because its elevation goes along with increasing tumor grade and stage $[4,12]$. Moreover, PDE5 inhibitors are able to enhance the chemotherapeutic efficacy of anticancer drugs in different tumors and cancer cell types [9, 13-15] and inhibition of PDE5 has an impact on the attenuation of multidrug resistance, one of the major causes of unsuccessful anticancer treatments $[16,17]$.

Despite the tremendous growth of preclinical and clinical studies on the use of PDE5 inhibitors and their promising results, the mechanisms of action are in most cases not fully understood and detailed insights are needed. For example, the currently available data are insufficient to make any conclusive statement regarding the exact role of the PDE5 enzyme in cancer pathogenesis $[12,18]$. 
The aim of our research is the development of a PDE5-specific radioligand to support in vitro and in vivo studies on the investigation of pathology-specific changes in the availability of the enzyme using PET. Regarding tumor imaging, those studies would enable the assessment of the spatiotemporal pattern of PDE5 expression. Thereby, our particular interest is dedicated to a future investigation of brain tumors by characterizing the expression of PDE5 in healthy and diseased brain.

In recent years, several attempts have been made to develop PET radioligands targeting the PDE5 enzyme as reviewed elsewhere $[19,20]$. Most of the radiotracers are labeled with carbon-11 and suffer from a lack of specificity [21, 22]. Chekol et al. reported on the development of fluorine-18 labeled PDE5 radioligands $[23,24]$. In particular a vardenafilbased derivative showed a high retention in the lungs which was interpreted to be PDE5specific because of the high expression of PDE5 in this organ [23]. Biodistribution studies in mice showed that none of the ligands investigated in this study demonstrated significant brain uptake. By contrast, a recent report by the same group revealed that a pyridopyrazinone based ${ }^{18} \mathrm{~F}$-labeled derivative has shown accumulation in rat brain. Although the radiotracer readily entered the brain, the radioactivity uptake was not specific toward PDE5. On the other hand, this compound demonstrated specific binding in transgenic mice with cardiomyocytespecific PDE5 overexpression [24].

Searching for a suitable lead compound for the development of a brain penetrating PDE5 radioligand, we decided to focus our attention on the heterocyclic quinoline based compounds 1 and 2 (Figure 1). These derivatives were described with a high PDE5 potency and the potential to cross the blood-brain barrier $[25,26]$. Structure-activity relationship studies (SAR) revealed important structural features of this family of PDE5 inhibitors $[25,26]$. The quinoline scaffold bearing a hydroxymethyl group at position C-3 ("east region") improves the inhibitory activity on PDE5 and the solubility compared to the other core ring systems tested. The binding to the protein is highly dependent on the presence of the secondary amino functionality at position C-4 ("north region") [27]. Moreover, the influence of a combination of different substituents on positions C- 6 and C-8 of the quinoline scaffold was studied resulting in the so far most potential candidates 1 [25] and 2 [26]. Based on these results, we started our attempts to introduce a fluorine containing structural moiety at the "east region" of 1 and 2 by substituting the hydroxymethyl group on C-3 by a fluoroethyl and fluoroethoxymethyl group [28], resulting in the development of the new PDE5 radioligand 
$\left[{ }^{18} \mathrm{~F}\right]$ ICF24027 [29]. However, due to the fast formation of a brain-penetrable radiometabolite, this radiotracer was not suitable for PDE5 imaging in brain. The aim of the present study was to investigate the possibility of introducing fluorine containing moieties at the "north" or "south region" (position 4 or 8 ) of the quinoline scaffold (Figure 1) in order to develop a specific and metabolically more stable ${ }^{18} \mathrm{~F}$-labeled radioligand for imaging of the PDE5 enzyme in brain.

\section{Results and discussion}

\subsection{Ligand development}

\subsubsection{Concept}

For introduction of the fluorine atom at the "north region" of the lead structures $\mathbf{1}$ and $\mathbf{2}$, modification of the chlorobenzene scaffold was intended (Figure 1). As the secondary amine substituted at the C-4 position of the quinoline core is known to be crucial for the PDE5 inhibitory activity [27], it was not considered for an alkylation with a fluorinated alkyl side chain. In order to enable a straightforward radiofluorination, the substitution of a 2-fluoro-3methoxypyridine ring was favored (3 and $\mathbf{4}$ ). This strategy allows nucleophilic heteroaromatic substitution with $\left[{ }^{18} \mathrm{~F}\right]$ fluoride using synthetic easily accessible halogen or nitro substituted precursor compounds [30].

Structural modifications performed at the "south region" of the lead compound 1 usually have a minor impact on the PDE5 inhibitory activity [26]. Therefore, we selected four structurally diverse moieties to introduce the fluorine at the C-8 position of the quinoline core by substituting the ethyl group of 1 as follows (Figure 1): (i) fluoropropyl alkyl chain (5), (ii) 4fluoropiperidine (6) and (iii) 3-fluoroazetidine (7) as aliphatic rings and (iv) 2-fluoropyridines (8 and $\mathbf{9})$ as aromatic rings. 


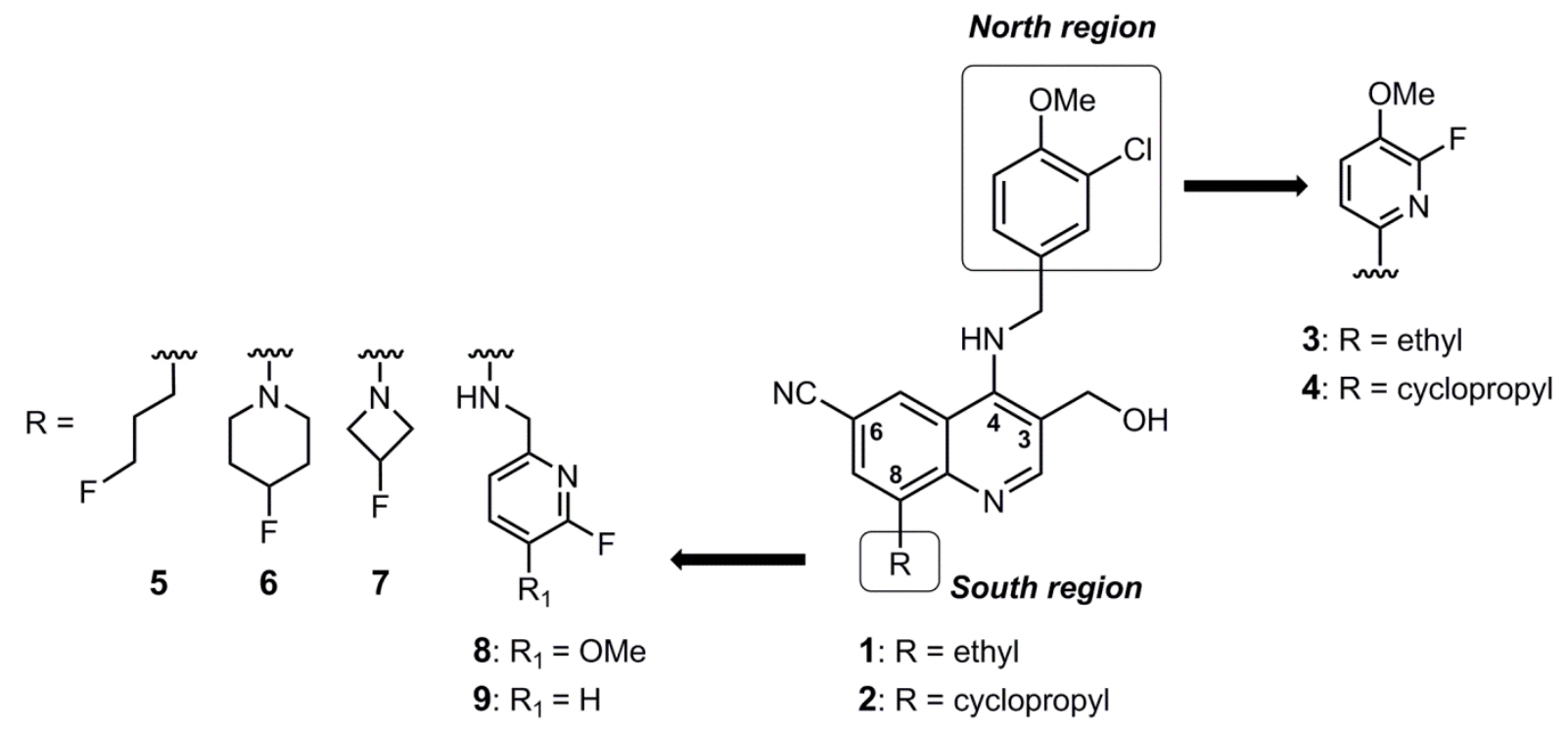

Figure 1. Design of fluorinated compounds with introduction of the fluorine atom on substituents located at C-4 or C-8 position of the quinoline core of the lead compounds $\mathbf{1}$ and $\mathbf{2}$.

\subsubsection{Organic Chemistry}

The synthesis of derivatives $\mathbf{3}$ and $\mathbf{4}$ bearing modifications in the "north region" of the quinoline scaffold, started with the coupling of amine 10a with the 4-chloroquinoline 11 via aromatic nucleophilic substitution at the C-4 position of the quinoline ring (Scheme 1). 4Chloroquinoline 11 was prepared as previously described by our group [28]. The necessary amine 10a was synthesized in 5 steps according to known procedures (see supporting information). The resulting derivative 12 was further functionalized at the C-8 position via a Suzuki-Miyaura cross-coupling reaction with commercially available ethylboronic or cyclopropylboronic acid to generate $13 \mathrm{a}$ and $13 \mathrm{~b}$ with a yield of $68 \%$ and $86 \%$, respectively. The desired fluorinated compounds $\mathbf{3}$ and $\mathbf{4}$ were obtained by a selective reduction of the ester function with lithium tri-tert-butoxyaluminum hydride (LTBA). 


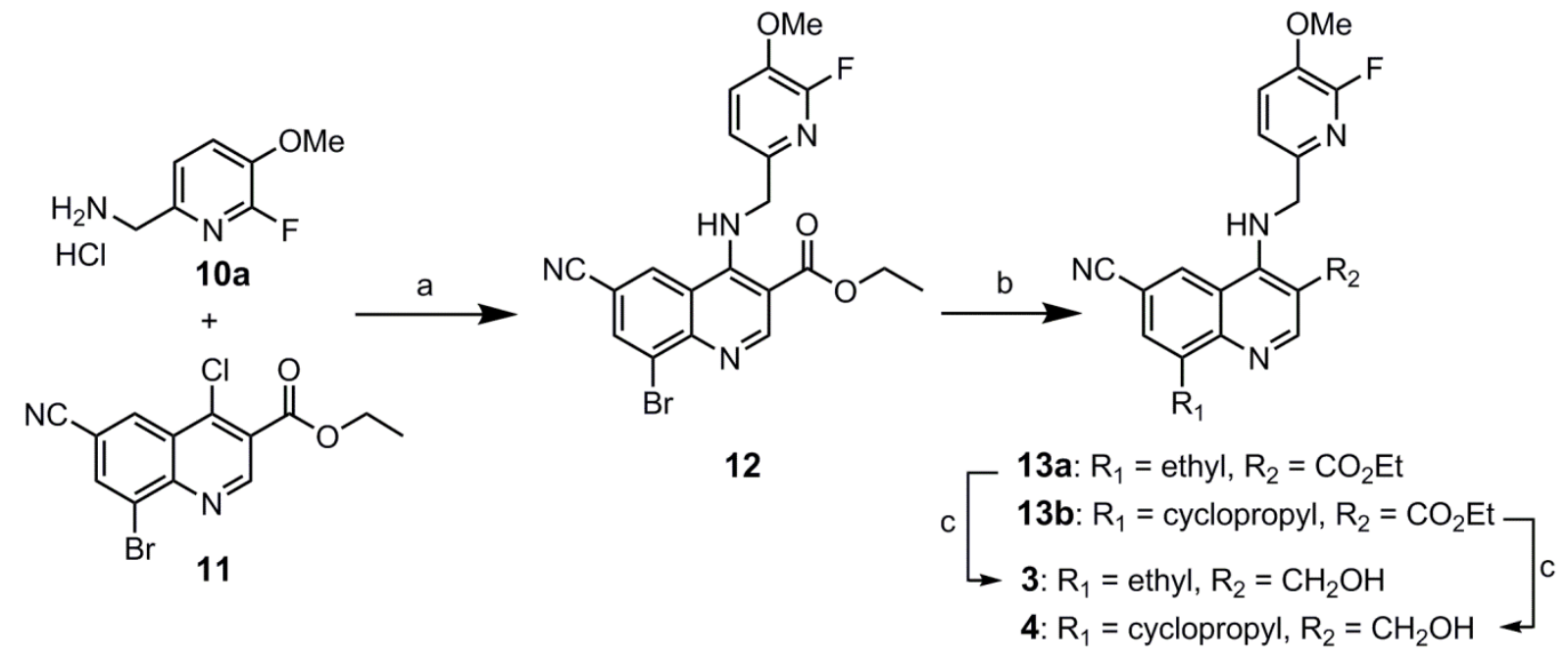

Scheme 1. Synthesis of "north region" compounds $\mathbf{3}$ and $\mathbf{4}$.

Reagents and conditions: (a) 10a, N,N-diisopropylethylamine (DIPEA), $n$-propanol, reflux, 2 h; (b) ethylboronic acid for $13 a$ and cyclopropylboronic acid for $13 \mathrm{~b}, \mathrm{Pd}\left(\mathrm{PPh}_{3}\right)_{4}, \mathrm{Cs}_{2} \mathrm{CO}_{3}$, toluene, reflux, 15-22 $\mathrm{h}$; (c) LTBA, THF, $50^{\circ} \mathrm{C}$, $30 \mathrm{~h}$.

For the synthesis of compounds 5-9 modified at the C-8 position of the quinoline core, two different palladium-catalyzed reaction pathways were developed in order to form the C-C bond in 5 and the $\mathrm{C}-\mathrm{N}$ bond in 6-9 starting from compound 14 [28]. The synthesis of 5 started with the reaction of allyl alcohol with 9-borabicyclo[3.3.1]nonane (9-BBN) in tetrahydrofuran to afford the corresponding boronic ester, generated in situ at the $\alpha$-position of the alkene reactant. This complex was used directly for the subsequent Suzuki-Miyaura cross-coupling reaction with 14 (Scheme 2, strategy 1) to afford the alcohol 15 with a yield of $26 \%$. It is worth mentioning, that the concomitant formation of the debrominated analog of $\mathbf{1 4}$ was observed in a ratio of about $1 / 1$.

To further convert the alcohol function into fluorine, $\mathbf{1 5}$ was treated by diethylaminosulfur trifluoride (DAST) under classical conditions. However, the formation of the desired fluorinated product 16 could not be observed and only a by-product, identified as compound 17, was obtained with a yield of $57 \%$. We assume that the formation of this stable six-membered ring in $\mathbf{1 7}$ is a result of an intramolecular cyclization caused by the electron donor effects of the free secondary amine located at the C-4 position of the quinoline, as previously observed during the chemical modifications at the "east region" of this compound class [28]. Therefore, an alternative strategy (Scheme 2, strategy 2) was developed, in which the amine at the C-4 position of 14 was beforehand protected using di-tert-butyl dicarbonate $\left(\mathrm{Boc}_{2} \mathrm{O}\right)$ in the presence of catalytic amounts of $\mathrm{N}, \mathrm{N}$-4-dimethylaminopyridine (DMAP) to obtain 18 with a 
yield of $74 \%$. For the following Suzuki-Miyaura coupling at C-8 position, a fluorinated boronic ester was used, generated from the reaction of allyl bromide with tetra- $n$-butylammonium fluoride (TBAF) and 9-BBN. Thus, the fluoroalkylated compound 19 was successfully synthesized and used in the next synthesis step without further purification. Finally, the ester group was converted into the corresponding alcohol to obtain $\mathbf{2 0}$ which was treated with trifluoroacetic acid (TFA) to remove the Boc protecting group resulting in the desired derivative 5 with a yield of $31 \%$.<smiles>[R7]OC(=O)O[R16](=O)[O-]</smiles>

Scheme 2. Synthesis of "south region" compound 5.

Reagents and conditions: (a) 1) Allyl alcohol, 9-borabicyclo[3.3.1]nonane (9-BBN), THF, $0{ }^{\circ} \mathrm{C} \rightarrow \mathrm{rt}, 7 \mathrm{~h} ; 2$ ) $\mathrm{Pd}\left(\mathrm{PPh}_{3}\right)_{4}, \mathrm{~K}_{2} \mathrm{CO}_{3}, \mathrm{H}_{2} \mathrm{O}, \mathrm{DMF}, 80^{\circ} \mathrm{C}, 22 \mathrm{~h}$; (b) Diethylaminosulfur trifluoride (DAST), $\mathrm{CH}_{2} \mathrm{Cl}_{2},-60{ }^{\circ} \mathrm{C} \rightarrow-30{ }^{\circ} \mathrm{C}, 2 \mathrm{~h}$; (c) (Boc) ${ }_{2} \mathrm{O}, \mathrm{DMAP}, \mathrm{Et}_{3} \mathrm{~N}, \mathrm{THF}, \mathrm{rt}, 2.5 \mathrm{~h}$; (d) 1) Allyl bromide (reagent and solvent), TBAF, rt, $30 \mathrm{~min} ; 2$ ) 9-BBN, THF, $\left.-10{ }^{\circ} \mathrm{C} \rightarrow \mathrm{rt}, 7 \mathrm{~h} ; 3\right) \mathrm{Pd}\left(\mathrm{PPh}_{3}\right)_{4}, \mathrm{~K}_{2} \mathrm{CO}_{3}, \mathrm{H}_{2} \mathrm{O}$, DMF, $80{ }^{\circ} \mathrm{C}, 16 \mathrm{~h}$; (e)LTBA, THF, $60{ }^{\circ} \mathrm{C}, 20 \mathrm{~h}$; (f) TFA $/ \mathrm{CH}_{2} \mathrm{Cl}_{2}, 1 / 2$, $\mathrm{v} / \mathrm{v}, \mathrm{rt}, 3 \mathrm{~h}$.

For the synthesis of compounds 6-9 via C-N bond formation at the C-8 position of compound 14 (Scheme 3), the Buchwald-Hartwig coupling reaction was used. Therefore, 14 was treated with commercially available 4-fluoropiperidine, 3-fluoroazetidine and the synthesized 
pyridines $\mathbf{1 0 a}$, b (see supporting information) to afford $\mathbf{2 1 a - d}$ in $56-93 \%$ yields. Further reduction of the ester function to the corresponding alcohol with LTBA provided the desired fluorinated compounds 6-9.<smiles>CCOC(=O)c1cnc2c(Br)cc(C#N)cc2c1NCc1ccc(OC)c(Cl)c1</smiles>

14<smiles>[R]c1cc(C#N)cc2c(NCc3ccc(OC)c(Cl)c3)c(C(=O)OCC)cnc12</smiles>

21a-d<smiles>[R]c1ccc(CNc2c(CO)cnc3c([R])cc(C#N)cc23)cc1Cl</smiles>

6-9

21a: $R=4$-fluoropiperidin-1-yl

21b: $R=3$-fluoroazetidin-1-yl

21c: $R=$ [(6-fluoro-5-methoxypyridin-2-yl)methyl]amino

21d: $R=$ [(6-fluoropyridin-2-yl)methyl]amino

Scheme 3. Synthesis of "south region" compounds 6-9.

Reagents and conditions: (a) 4-fluoropiperidine hydrochloride for 21a, 3-fluoroazetidine hydrochloride for 21b, 10a for 21a and $10 \mathrm{~b}$ for $21 \mathrm{~b}, \mathrm{Pd}(\mathrm{OAC})_{2}, \mathrm{BINAP}, \mathrm{Cs}_{2} \mathrm{CO}_{3}$, toluene, $120^{\circ} \mathrm{C}, 3 \mathrm{~h}$ for $21 \mathrm{a}$ and $\mathrm{b}, 16 \mathrm{~h}$ for $21 \mathrm{c}$ and $4 \mathrm{~h}$ for 21d; (b) LTBA, THF, $60^{\circ} \mathrm{C}, 20 \mathrm{~h}$.

\subsection{Determination of inhibitory activity}

The inhibitory potential of the new fluorinated derivatives 3-9 on the human recombinant PDE5A1 protein and selected other human PDEs were evaluated by using an enzyme assay [31] with sildenafil as standard reference compound. The tests were performed at two different concentrations of the compounds; $100 \mathrm{nM}$ for PDE5A1 and $1.0 \mu \mathrm{M}$ for the other PDEs. The results are summarized in Table 1. Analyses of the data revealed a considerable decrease of PDE5A1 potency for derivatives 3 and 4 (34.6 and 20.9\% of inhibition, respectively) compared to the lead compounds 1 and 2 (90.3 and 84.2\%, respectively). Hence, the substitution of the 3-chloro-4-methoxy phenyl ring by a 2-fluoro-3-methoxy pyridine ring in the "north region" of the molecule is unfavorable for binding to the PDE5A1 protein. By contrast, derivatives 5-9 are characterized by structural modifications in the "south region" at $\mathrm{C} 8$ of the quinoline core. Although the introduced motifs are structurally quite diverse, their influence on the inhibitory activity toward the PDE5A1 is rather low and the obtained values do not differ considerably compared to $\mathbf{1}$ and $\mathbf{2}$. This is in accordance with the results reported 
by Fiorito et al. [26], who also modified the functionalities at the C8-position. All new derivatives 3-9 demonstrated a high selectivity for the PDE5 enzyme as shown by the weak inhibitory potential against PDE2A3, PDE3A, PDE4A1 and C2, PDE6AB, PDE9A1, PDE10A1, and PDE11A1 at $1 \mu \mathrm{M}$ concentrations.

As derivatives 6 and $\mathbf{7}$ showed the highest inhibition values for PDE5A1, they were selected for determination of $\mathrm{IC}_{50}$ values by measuring the inhibition at seven different concentrations $(0.25-1000 \mathrm{nM})$ [31]. With an $\mathrm{IC}_{50}$ value of $5.92 \mathrm{nM}$ compound 7 showed the highest inhibitory potential which is comparable to sildenafil $\left(\mathrm{IC}_{50}=6.23 \mathrm{nM}\right)$. Although the inhibitory activity of $\mathbf{7}$ is slightly lower compared to our first developed radiotracer [ ${ }^{18} \mathrm{~F}$ ]ICF24027 [28, 29], this compound demonstrated a good selectivity toward the other PDEs and was therefore selected for ${ }^{18} \mathrm{~F}$-labeling to generate a potential radiotracer for PET imaging of PDE5A.

Table 1. Percentage inhibition values of synthesized compounds against selected PDEs

\begin{tabular}{|c|c|c|c|c|c|c|c|c|c|c|}
\hline \multirow{2}{*}{ Comp. } & \multicolumn{9}{|c|}{ Percentage of inhibition (\%) } & \multirow{2}{*}{$\begin{array}{c}\mathrm{IC}_{50}(\text { PDE5A1) } \\
(\mathrm{nM})\end{array}$} \\
\hline & PDE2A3 $^{a}$ & PDE3A ${ }^{a}$ & PDE4A1 $^{\mathrm{a}}$ & PDE4C2 $^{\mathrm{a}}$ & PDE5A1 $^{\mathrm{b}}$ & PDE6AB $^{\mathrm{a}}$ & PDE9A1 $^{\mathrm{a}}$ & PDE10A1 $^{\text {a }}$ & PDE11A1 $^{a}$ & \\
\hline $1^{c}$ & 22.4 & $\mathrm{NI}$ & 45.4 & 40.9 & 90.3 & 49.8 & $\mathrm{NI}$ & 40.0 & 27.2 & \\
\hline $2^{c}$ & 15.3 & $\mathrm{NI}$ & 36.9 & 22.4 & 84.2 & 32.4 & $\mathrm{NI}$ & 22.9 & 24.1 & \\
\hline 3 & 10.4 & $\mathrm{NI}$ & 33.4 & 4.52 & 34.6 & 35.9 & 2.87 & 27.9 & 11.8 & n.d. \\
\hline 4 & 14.1 & $\mathrm{NI}$ & 22.2 & 15.9 & 20.9 & 23.2 & $\mathrm{NI}$ & 15.8 & 8.80 & n.d. \\
\hline 5 & 16.0 & $\mathrm{NI}$ & 37.3 & 18.6 & 80.7 & $\mathrm{NI}$ & $\mathrm{NI}$ & 46.8 & 16.4 & n.d. \\
\hline 6 & 17.0 & $\mathrm{NI}$ & 36.8 & 35.3 & 87.8 & 24.7 & 20.6 & 17.4 & 23.7 & 25.6 \\
\hline 7 & 18.9 & $\mathrm{NI}$ & 18.3 & 16.1 & 90.3 & 10.8 & 8.71 & 23.5 & 13.2 & 5.92 \\
\hline 8 & 76.6 & $\mathrm{NI}$ & 28.6 & 15.3 & 64.2 & 13.9 & 23.4 & 17.2 & 8.82 & n.d. \\
\hline 9 & 39.5 & $\mathrm{NI}$ & 24.5 & 18.7 & 80.5 & 5.79 & $\mathrm{NI}$ & 21.0 & 11.2 & n.d. \\
\hline ICF24027 & 7.49 & 18.2 & 9.03 & 32.4 & 88.4 & 60.4 & $\mathrm{NI}$ & 14.1 & 5.21 & 1.86 \\
\hline Sildenafil ${ }^{c}$ & 67.7 & 63.9 & 65.8 & 55.4 & 86.7 & 65.7 & 74.0 & 74.6 & 78.5 & 6.23 \\
\hline
\end{tabular}

a The compounds were tested at $1 \mu \mathrm{M}$ against PDE2A3, PDE3A, PDE4A1, PDE4C2, PDE6AB, PDE9A1, PDE10A1 and PDE11A1 and ${ }^{\mathrm{b}}$ at $100 \mathrm{nM}$ against human PDE5A1. NI means no inhibition; n.d. means not determined. ${ }^{\mathrm{C}}$ Own reported data [28].

\subsection{Precursor synthesis and radiochemistry}

For the synthesis of the new radioligand $\left[{ }^{18} \mathrm{~F}\right] 7$, the aliphatic nucleophilic substitution reaction of a precursor with a suitable leaving group by $\left[{ }^{18} \mathrm{~F}\right]$ fluoride was intended. As ${ }^{18} \mathrm{~F}$-labeling of an azetidine ring was only described in a single report [32], and the radiolabeling at a 
secondary carbon atom is usually more challenging than on a primary carbon atom [33], we investigated the use of two sulfonate leaving groups with different reactivity. Therefore, the precursor molecules $\mathbf{2 7}$ and $\mathbf{2 8}$ with methylbenzenesulfonate (tosylate) and 4nitrobenzenesulfonate (nosylate), respectively, were synthesized. According to the literature, the nosylate is known to be slightly more reactive than tosylate [34, 35].

Based on former experiments with this compound class (data not shown), the alcohol functionality was not protected as its acidity is expected to be too low to considerably influence the radiolabeling reaction.

\subsubsection{Synthesis of the precursor compounds}

The precursor compounds $\mathbf{2 7}$ and $\mathbf{2 8}$ were prepared as shown in Scheme 4. In a first step, the ester function of $\mathbf{1 4}$ was reduced to the corresponding alcohol $\mathbf{2 2}$, which was further protected as a silyl ether group to give $\mathbf{2 3}$. As described above for compound $\mathbf{2 1 \mathbf { b }}$, the azetidine moiety was then coupled on the C-8 position of the quinoline core of derivative $\mathbf{2 3}$ using freshly prepared azetidin-3-ol trifluoroacetate [36] to produce compound 24 with a yield of 51\%. Afterwards, the tosylation and nosylation reactions were performed under classical conditions to afford $\mathbf{2 5}$ and 26, respectively. Finally, the triisopropyl silyl (TIPS) protecting group was cleaved in the presence of TBAF to afford the desired tosylate $\mathbf{2 7}$ and nosylate $\mathbf{2 8}$ precursors with yields in the range of $60-70 \%$.
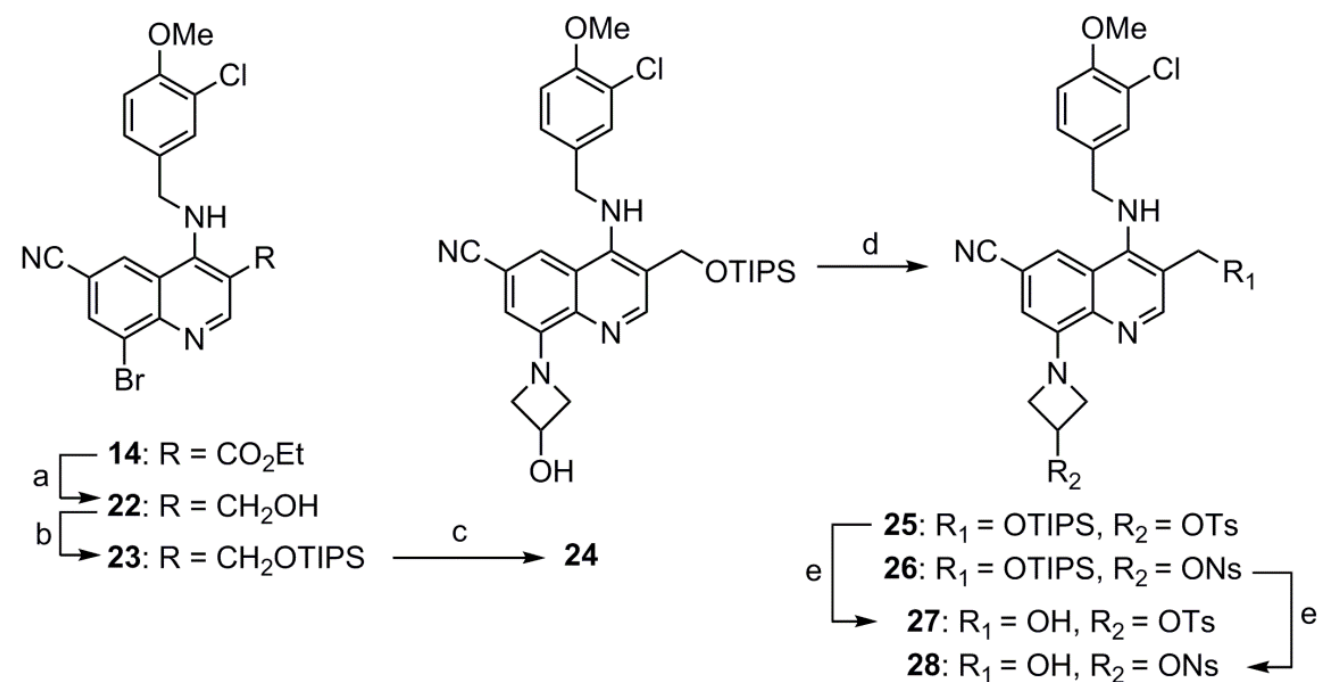

Scheme 4. Synthesis of precursor compounds $\mathbf{2 7}$ and 28 for ${ }^{18} \mathrm{~F}$-radiolabeling.

Reagents and conditions: (a) LTBA, THF, $60{ }^{\circ} \mathrm{C}, 20 \mathrm{~h}$, (b) TIPSCl, Et 3 N, DMAP, DMF, rt, 36 h, (c) azetin-3-ol trifluoroacetate, $\mathrm{Cs}_{2} \mathrm{CO}_{3}, 2$,2'-bis(diphenylphosphino)-1,1'-binaphthyl (BINAP), $\mathrm{Pd}(\mathrm{OAc}$ ), toluene, reflux, $3 \mathrm{~h}$, (d) TsCl for 25 or $\mathrm{NsCl}$ for 26, Et $3 \mathrm{~N}, \mathrm{DMAP}, \mathrm{DCM}, \mathrm{rt}, 19 \mathrm{~h},(\mathrm{e}) \mathrm{TBAF}, \mathrm{THF}, \mathrm{rt}, 30 \mathrm{~min}$. 


\subsubsection{Radiosyntheses}

\subsubsection{Methylbenzenesulfonate (OTs) as leaving group}

The ${ }^{18} \mathrm{~F}$-labeling of the tosylate precursor $\mathbf{2 7}$ was investigated with the conventional potassium carbonate $\left(\mathrm{K}_{2} \mathrm{CO}_{3}\right)$ - kryptofix-222 system $\left(\mathrm{K}\left[{ }^{18} \mathrm{~F}\right] \mathrm{F} / \mathrm{K}_{222}\right.$ complex) changing different reaction parameters (solvents, temperatures and reaction times). When the reaction was performed in acetonitrile (ACN) at $90^{\circ} \mathrm{C}$ and in DMF at 130 or $150{ }^{\circ} \mathrm{C}$, only the formation of by-products could be observed. Using DMSO at $130{ }^{\circ} \mathrm{C}$ resulted in the generation of $\left[{ }^{18} \mathrm{~F}\right] 7$ with a radiochemical yield of $2-3 \%$ after 5 min (determined by radio TLC of a sample of the reaction mixture). However, the RCY decreased with increasing reaction time indicating a decomposition of the radiotracer. The same effect was observed for the precursor $\mathbf{2 7}$ itself, which was entirely decomposed after 15 min reaction time according to HPLC analysis, probably due to a competing ß-elimination reaction. Further investigations revealed that 100 ${ }^{\circ} \mathrm{C}$ was the optimal temperature for this radiolabeling system resulting in RCYs of $3-4 \%$ after $15 \mathrm{~min}$ (Scheme 5). The reactions were performed with a precursor amount of $2.0-2.5 \mathrm{mg}$; higher concentrations of $\mathbf{2 7}$ did not result in an increase of RCY. Due to the presumed base sensitivity of both, the precursor and the radiotracer, potassium oxalate as less basic counter ion was tested in combination with $\mathrm{K}_{222}$ in DMSO. However, these conditions did not lead to the formation of $\left[{ }^{18} \mathrm{~F}\right] 7$. Moreover, we tested $\left[{ }^{18} \mathrm{~F}\right]$ TBAF as a mild nucleophilic ${ }^{18} \mathrm{~F}$-fluorination agent in tert-amyl alcohol, as previously used by Zhang et al. for radiolabeling of the same azetidine motif in a PDE2A radioligand [32]. However, in contrast to the reported results (RCY of $7.3 \%$ at the end of the synthesis) we could not obtain the desired radiotracer $\left.{ }^{[18} \mathrm{F}\right] 7$ under these conditions.

Finally, it turned out that using the $\mathrm{K}\left[{ }^{18} \mathrm{~F}\right] \mathrm{F} / \mathrm{K}_{222}$ complex in DMSO was most useful for the synthesis of $\left[{ }^{18} \mathrm{~F}\right] 7$ on the basis of a tosylate precursor and was therefore translated to an automated procedure using the TRACERlab FX2 $\mathrm{N}$ synthesis module (GE Healthcare). The synthesizer setup is described in the experimental part. Briefly, after trapping and elution of $\left[{ }^{18} \mathrm{~F}\right]$ fluoride by an aqueous potassium carbonate solution from an anion exchange cartridge, the labeling reaction of the azeotropically dried $\mathrm{K}\left[{ }^{18} \mathrm{~F}\right] \mathrm{F} / \mathrm{K}_{222}$ complex with the precursor 27 was performed in DMSO for $15 \mathrm{~min}$ at $100{ }^{\circ} \mathrm{C}$. For isolation of $\left[{ }^{18} \mathrm{~F}\right] 7$, the crude reaction mixture was diluted with aqueous acetonitrile and directly applied to a semi-preparative HPLC system (chromatogram in supporting information). The radiotracer fraction was collected at a 
retention time of 22 - 24 min and purified by solid phase extraction (SPE) using a C18-cartridge. The obtained radiotracer eluate was transferred out of the hot cell, concentrated and formulated in sterile isotonic saline containing $10 \%$ of ethanol. In a total synthesis time of 80 min, $\left[{ }^{18} \mathrm{~F}\right] 7$ could be reproducibly produced with a high radiochemical purity of $\geq 99 \%$, a radiochemical yield of $2.5 \pm 0.1 \%(n=3)$ and molar activities between 14 and $28 \mathrm{GBq} / \mu \mathrm{mol}(\mathrm{n}$ $=3$, end of synthesis EOS) with starting activities of $4-5 \mathrm{GBq}$. Co-injection of the corresponding reference compound $\mathbf{7}$ confirmed the identity of $\left[{ }^{18} \mathrm{~F}\right] 7$ by analytical radio-HPLC (chromatogram in supporting information).

\subsubsection{4-Nitrobenzenesulfonate (ONs) as leaving group}

For ${ }^{18} \mathrm{~F}$-labeling of the nosylate precursor $\mathbf{2 8}$ we started as for the tosylate by using the $\mathrm{K}\left[{ }^{18} \mathrm{~F}\right] \mathrm{F} / \mathrm{K}_{222}$ complex. Reactions were performed in $\mathrm{ACN}, \mathrm{DMF}$ and DMSO at different temperatures $\left(90^{\circ} \mathrm{C}\right.$ for $\mathrm{ACN}, 130$ and $150^{\circ} \mathrm{C}$ for DMF and DMSO, resp.) resulting in RCYs lower than $1 \%$. Moreover, the tertiary alcohols tert-BuOH and 3-methyl-3-pentanol (3M3P) were tested, because nonpolar protic solvents are described to be beneficial for aliphatic nucleophilic substitution $[37,38]$ as e.g. reported for $\left[{ }^{18} \mathrm{~F}\right] \mathrm{FLT}[39,40]$ and $\left[{ }^{18} \mathrm{~F}\right] \mathrm{FES}[41]$. 3Methyl-3-pentanol (3M3P) was recently used by Marchand et al. [40] because of its high boiling point of $123{ }^{\circ} \mathrm{C}$ avoiding an excessive pressure in the reactor. For the $\left[{ }^{18} \mathrm{~F}\right] \mathrm{FLT}$ synthesis, the ratio of nosylate precursor to base was also shown to be crucial for the labeling process $[40,42]$. In the presence of an excess amount of base, the precursor is undergoing an E2 elimination mechanism, thus reducing the desired nucleophilic ${ }^{18} \mathrm{~F}$-fluorination [42]. Therefore, we started with a constant precursor to base ratio of 1.5 using $4 \mathrm{mg}$ of precursor 28 and $0.6 \mathrm{mg}$ of $\mathrm{K}_{2} \mathrm{CO}_{3}$ as base. While reaction in tert- $\mathrm{BuOH}$ at $95^{\circ} \mathrm{C}$ resulted in an $\mathrm{RCY}$ of $2 \%$, a higher $\mathrm{RCY}$ of $7 \%$ could be achieved in $3 \mathrm{M} 3 \mathrm{P}$ at $125^{\circ} \mathrm{C}$. With the aim to minimize the amount of precursor used, we further investigated the 3M3P system and found that a minimal ratio of 0.75 ( $2 \mathrm{mg}$ precursor and $0.6 \mathrm{mg}$ base) is necessary to achieve this RCY. However, when transferring this procedure to an automated process, we observed an insufficient elution of $\left[{ }^{18} \mathrm{~F}\right]$ fluoride from the anion exchange cartridge with this low amount of base. To reduce the loss of activity on the cartridge to a minimum of $10 \%, 1.2 \mathrm{mg}$ of $\mathrm{K}_{2} \mathrm{CO}_{3}$ has been found to be necessary in our automated system, which would result in the undesired use of higher precursor amounts. 
Because of the observed base sensitivity of the nosylate precursor $\mathbf{2 8}$, [ $\left.{ }^{18} \mathrm{~F}\right]$ TBAF was also investigated as fluorination agent. While in DMSO no radiolabeling could be obtained, an RCY of up to $10 \%$ was found in $3 \mathrm{M} 3 \mathrm{P}$ at $125^{\circ} \mathrm{C}$ with $2 \mathrm{mg}$ of 28 . By chance we observed that small amounts of water favored this radiolabeling reaction. The presence of water in ${ }^{18} \mathrm{~F}$-fluorination reactions can positively influence the RCY as already described by different groups [43, 44] and has been explained with the dependence of the nucleophilicity of fluoride by its hydration state $[45,46]$. Indeed, in our system the addition of $50-100 \mu \mathrm{L}$ water to the reaction mixture (total volume $1 \mathrm{~mL}$ ) increased the RCY up to $20 \%$ (Scheme 5). However, the addition of more water lead to a reduction of the RCY. Attempts to skip the azeotropic drying step by eluting the $\left[{ }^{18} \mathrm{~F}\right]$ fluoride with tetra- $n$-butylammonium hydrogen carbonate $\left(\mathrm{TBAHCO}_{3}\right)$ dissolved in the radiolabeling solvent $3 \mathrm{M} 3 \mathrm{P}$ and $100 \mu \mathrm{L}$ water failed due to the immiscibility of this tertiary alcohol and water.

Finally, the conditions were applied to an automated procedure using the TRACERlab FX2 N module. The detailed procedure is described in the experimental part. Briefly, after trapping on an anion exchange cartridge, the $\left.{ }^{18} \mathrm{~F}\right]$ fluoride was eluted with $\mathrm{TBAHCO}_{3}$ dissolved in an ACN/water solution and the resulting $\left[{ }^{18} \mathrm{~F}\right]$ TBAF was dried by azeotropic distillation. The radiolabeling reaction was performed after addition of the nosylate precursor $\mathbf{2 8}$ dissolved in $3 \mathrm{M} 3 \mathrm{P} / \mathrm{ACN} /$ water $(700 / 200 / 80, \mathrm{v} / \mathrm{v} / \mathrm{v})$ for $10 \mathrm{~min}$ at $125^{\circ} \mathrm{C}$. For isolation of $\left[{ }^{18} \mathrm{~F}\right] 7$ with semipreparative HPLC, the solvent was removed under reduced pressure because a strong peak broadening was observed when the tertiary alcohol remained in the injection solution. After evaporation, the crude reaction mixture was diluted with aqueous acetonitrile and applied to a semi-preparative HPLC system (chromatogram in supporting information). The radiotracer fraction was collected at a retention time of $26-29 \mathrm{~min}$ and purified by solid-phase extraction (SPE) using a C18 cartridge. The obtained radiotracer eluate was transferred out of the hot cell, concentrated and formulated in sterile isotonic saline containing $10 \%$ of ethanol. The entire process for this radiosynthesis lasted about $85 \mathrm{~min}$. Finally, $\left[{ }^{18} \mathrm{~F}\right] 7$ could be reproducibly produced with a high radiochemical purity of $\geq 99 \%$, a radiochemical yield of $4.9 \pm 1.5 \%$ ( $n=$ 3) and molar activities between 28 and $58 \mathrm{GBq} / \mu \mathrm{mol}(\mathrm{n}=3$, EOS) with starting activities of 4 $5 \mathrm{GBq}$. The identity of $\left[{ }^{18} \mathrm{~F}\right] 7$ was confirmed by analytical radio-HPLC (chromatogram in supporting information). 
<smiles>[M]Oc1ccc(CNc2c(CO)cnc3c(N4CC([R])C4)cc(C#N)cc23)cc1Cl</smiles>

27; $R=$ OTs

$28 ; \mathrm{R}=\mathrm{ONS}$

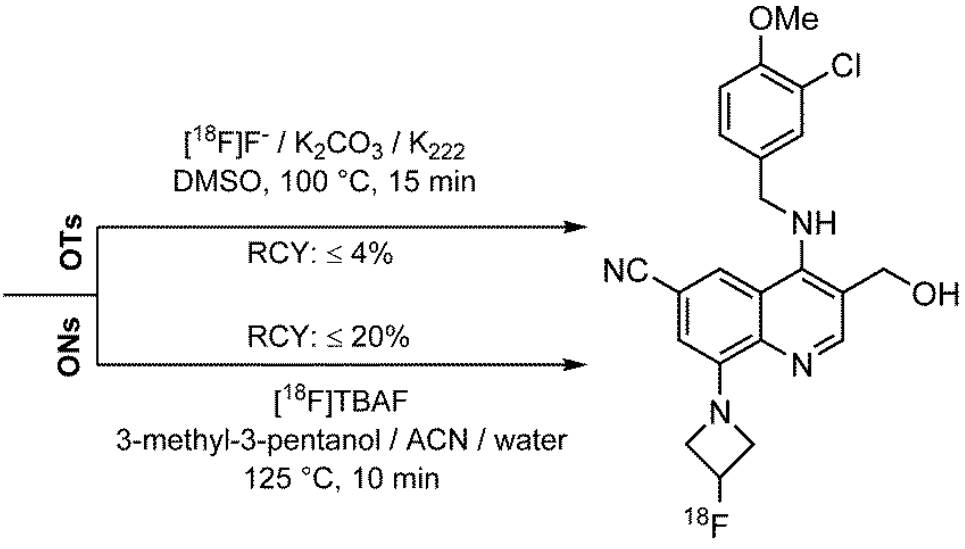

$\left[{ }^{18} \mathrm{~F}\right] 7$

Scheme 5. ${ }^{18} \mathrm{~F}$-Labeling procedures developed with the tosylate and nosylate precursors ( 27 and $\mathbf{2 8}$ ) to radiosynthesize the new PDE5 radioligand $\left[{ }^{18} \mathrm{~F}\right] 7$.

The stability of the radiotracer was investigated by incubation at $40{ }^{\circ} \mathrm{C}$ in phosphate-buffered saline (PBS) and pig plasma samples. $\left.{ }^{18} \mathrm{~F}\right] 7$ proved to be stable in these media, and no defluorination or degradation was observed within 60 min of incubation time.

\subsection{In vitro autoradiographic studies of $\left[{ }^{18} \mathrm{~F}\right] 7$ on porcine brain}

To investigate the distribution of binding sites of $\left[{ }^{18} \mathrm{~F}\right] 7$ in brain, in vitro autoradiographic studies on porcine brain slices were performed. The most dense binding was detected in the fornix (Fx), followed by corpus callosum (CC) and thalamus (Th). Although in human, PDE5 expression was demonstrated in cerebellum (Cb), cortex (Cx), and hippocampus (Hip) [47], only modest levels of binding were observed for $\left[{ }^{18} \mathrm{~F}\right] 7$ on these structures of the porcine brain.

To investigate the specificity of $\left[{ }^{18} \mathrm{~F}\right] 7$ binding, blocking studies with co-incubation of $1 \mu \mathrm{M}$ of 7 or $1 \mu \mathrm{M}$ of sildenafil were performed (data in Figure 2). Only a slight decrease of $\left.{ }^{[8} \mathrm{F}\right] 7$ binding in the range of $10-25 \%$ for all of the investigated brain regions was observed indicating a high non-specific binding of the radiotracer. This high non-specific binding has also been observed for our previously reported PDE5 radioligand [ $\left.{ }^{18} \mathrm{~F}\right]$ ICF24027 and we assumed it could be related to its high lipophilicity ( $\log D$ of 3.9 calculated using ACD/Labs) [29]. However, in comparison to $\left[{ }^{18} \mathrm{~F}\right]$ ICF24027, the hydroxyl group of $\left[{ }^{18} \mathrm{~F}\right] 7$ is non-alkylated resulting in a calculated $\log D$ value of 1.56 . Thus, the high non-specific binding might be caused by other properties. Probably with an $\mathrm{IC}_{50}$ value of $5.92 \mathrm{nM}$ as a measure of the inhibitory potential, 
the actual affinity of $\left[{ }^{18} \mathrm{~F}\right] 7$ is still too low for a specific imaging of PDE5 as recently discussed by P. Cumming [48]. Based on an estimation of $B_{\max }$ values in certain brain regions, the author proposed the need of ligands with sub-nanomolar affinity to achieve a binding potential suitable for PET imaging.

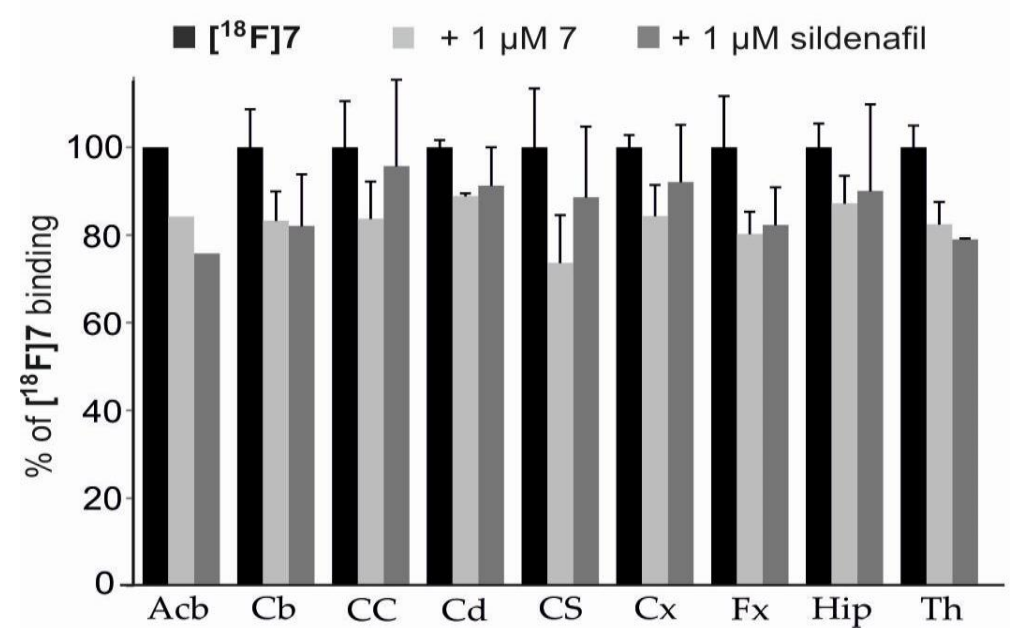

Figure 2. Binding of $\left[{ }^{18} \mathrm{~F}\right] 7(2.02 \pm 0.75 \mathrm{nM})$ in porcine brain in vitro $(\mathrm{n}=3)$. Remaining binding of $\left[{ }^{18} \mathrm{~F}\right] 7$ in the presence of $1 \mu \mathrm{M} 7$ or $1 \mu \mathrm{M}$ sildenafil is presented as percentage of total binding $(=100 \%)$. Abbreviations: $\mathrm{Acb}=$ Nucleus accumbens, $\mathrm{Cb}=$ Cerebellum, $\mathrm{CC}=$ Corpus callosum, $\mathrm{Cd}=$ Nucleus caudatus, $\mathrm{CS}=$ Colliculus superior, $\mathrm{Cx}$ $=$ Cortex, $\mathrm{Fx}=$ Fornix, Hip $=$ Hippocampus, Th $=$ Thalamus.

\subsection{In vivo studies of $\left[{ }^{18} \mathrm{~F}\right] 7$ in mouse}

\subsubsection{Organ uptake}

For mouse brain, we determined standard uptake values (SUV) of $1.10(n=1)$ and $0.08(n=2$; single values: 0.10 and 0.05$)$ at 5 and $30 \mathrm{~min}$, respectively, after injection of $\left[{ }^{18} \mathrm{~F}\right] 7$, which indicate perfusion-mediated uptake of the radioligand in the brain followed by a relatively fast washout of the activity. In periphery, we could detect the high initial uptake in organs with comparatively high expression of PDE5 such as lung (SUV $=8.70$ and 0.98 at 5 and $30 \mathrm{~min}$ p.i.) and heart (SUV $=5.00$ and 0.48 at 5 and $30 \mathrm{~min}$ p.i.) [49]. Although it might be assumed that the herein observed order of late-stage SUV with lung $>$ heart $>$ brain $(0.98,0.48,0.08$ at 30 min p.i.) in CD1 mouse indeed correlates with the reported mRNA levels in rabbit [49], this assumption remains to be investigated.

\subsubsection{Metabolism studies}


In vivo metabolism of $\left[{ }^{18} \mathrm{~F}\right] 7$ was studied in $\mathrm{CD}-1$ mice $(\mathrm{n}=2)$ by investigation of plasma and brain samples obtained from at $30 \mathrm{~min}$ after injection of the radioligand. Analysis of the samples was performed by the use of micellar chromatography (MLC) and RP-HPLC. MLC allows the direct injection of samples into the HPLC system without eliminating the tissue matrix due to the ability of micellar aggregates to dissolve the proteins and other components. For RP-HPLC analysis proteins were precipitated by treating aliquots of plasma and brain homogenates with a mixture of acetone/water, resulting in recoveries of about $97 \%$ of activity in the supernatants.

The results obtained with both methods correlated very well. According to the chromatograms (Figure 3), a high fraction of radiometabolites was detected in plasma with only $13 \%$ of total radioactivity representing the nonmetabolized radioligand $\left[{ }^{18} \mathrm{~F}\right] 7$. Several radioactive metabolites were observed and we assume that some of them are brain penetrable as they were also observed in the corresponding brain samples. Thus, only $24 \%$ of total activity was represented by intact $\left[{ }^{18} \mathrm{~F}\right] 7$ in brain (Figure 3 ). In particular, the more lipophilic radiometabolite $\left[{ }^{18} \mathrm{~F}\right] \mathrm{M} 4$ seems to easily penetrate the blood-brain barrier and accumulates in the brain. By contrast, the metabolites group $\left[{ }^{18} \mathrm{~F}\right] \mathrm{M} 1$ is very polar as the species are eluting without significant retention on the hydrophobic HPLC column. Despite this polarity, they were also detected in the brain samples to a considerable amount (Figure 3). We assume that this group of radiometabolites consists of small molecular weight species containing alcohol, aldehyde or acid functions which might be formed due to $\mathrm{N}$-dealkylation of the azetidine ring. However, as the radioligand described herein doesn't show the potential for future use, we didn't characterize the structure of the radiometabolites. 
A) MLC

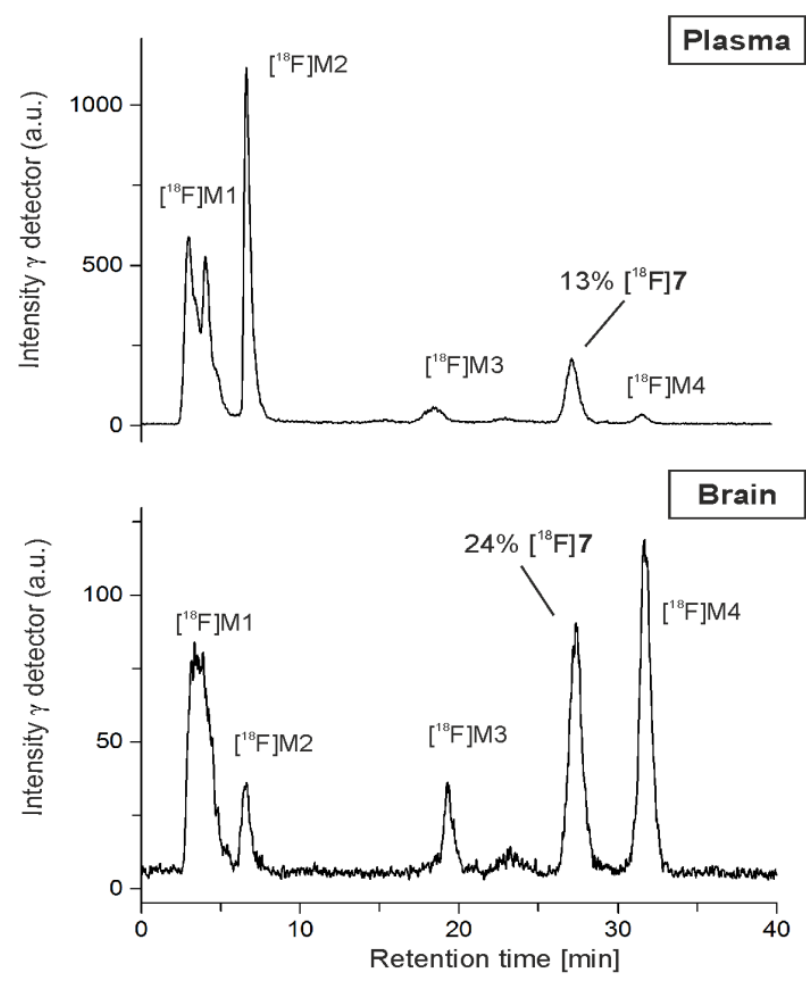

B) RP-HPLC
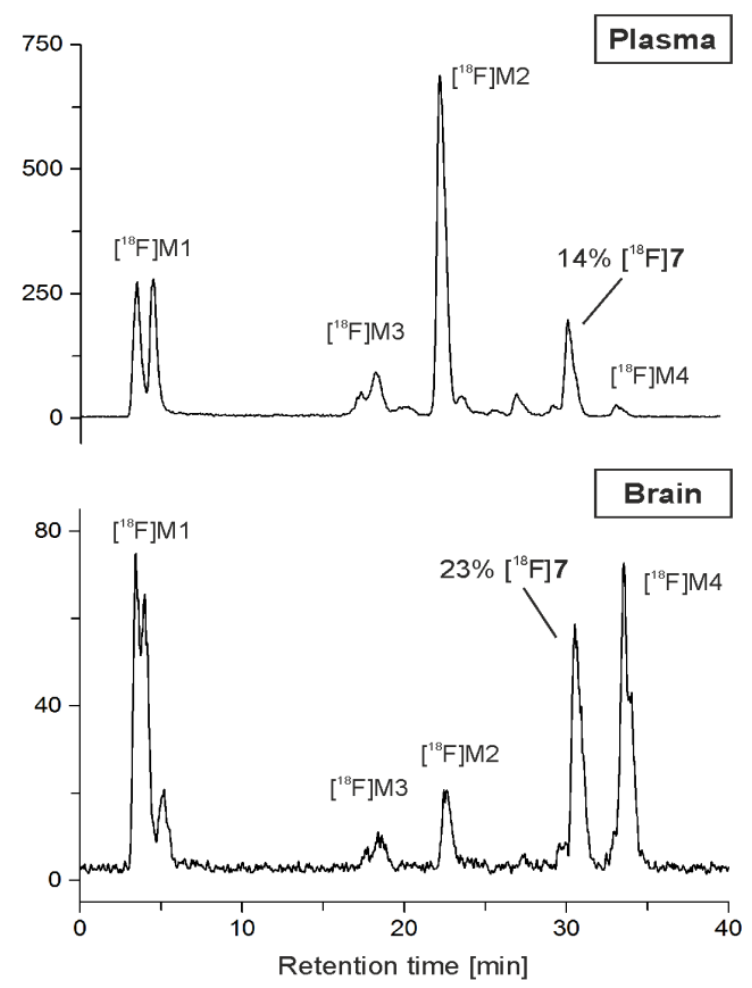

Figure 3. Exemplary MLC (A) and RP-HPLC (B) radio-chromatograms of a mouse plasma and brain sample at 30 minutes p.i. of $\left.{ }^{18} \mathrm{~F}\right] 7$ (conditions MLC: Reprosil-Pur C18-AQ, $250 \times 4.6 \mathrm{~mm}$, gradient with an eluent mixture of THF/100 mM SDS/10 mM Na $2 \mathrm{HPO}_{4}$ aq., $1.0 \mathrm{~mL} / \mathrm{min}$; conditions RP-HPLC: Reprosil-Pur C18-AQ, $250 \times 4.6 \mathrm{~mm}$, gradient with an eluent mixture of $\mathrm{ACN} / 20 \mathrm{mM} \mathrm{NH} \mathrm{H}_{4} \mathrm{OAc}$ aq., $1.0 \mathrm{~mL} / \mathrm{min}$ )

\section{Summary and conclusion}

Continuing our efforts in the development of PET imaging probes for characterization of the expression pattern of the cyclic nucleotide phosphodiesterase PDE5 in brain, we synthesized a series of fluorinated derivatives based on a quinoline lead compound (1). While structural modification in the "east and west regions" of 1 have been already investigated by our group [28], the present study was dedicated to modifications in the "north and south regions" of 1. Substitution of a 2-fluoro-3-methoxypyridine ring in the "north part" of 1 caused a loss of inhibitory potential on PDE5. By contrast, the "south part" of $\mathbf{1}$ is less restricted for structural modifications resulting in the potential 3-fluoroazetidine containing derivative $\mathbf{7}$ with an $\mathrm{IC}_{50}$ value of $5.92 \mathrm{nM}$ for PDE5 and high selectivity regarding other PDEs.

As ${ }^{18} \mathrm{~F}$-labeling of a secondary carbon is challenging, two different leaving groups were investigated for a nucleophilic aliphatic substitution with $\left[{ }^{18} \mathrm{~F}\right]$ fluoride. Interestingly, highest $\mathrm{RCY}$ of $4.9 \pm 1.5 \%$ (EOS) was achieved for automated radiosynthesis of $\left[{ }^{18} \mathrm{~F}\right] 7$ due to the 
addition of small amounts of water to the reaction mixture of the nosylate precursor in 3methyl-3-pentanol and $\left[{ }^{18} \mathrm{~F}\right]$ TBAF. By contrast, using the tosylate precursor and $\mathrm{K}\left[{ }^{18} \mathrm{~F}\right] \mathrm{F} / \mathrm{K}_{222}$ complex in DMSO resulted in lower RCY of $2.5 \pm 0.1 \%$ (EOS). First in vivo studies of $\left[{ }^{18} \mathrm{~F}\right] 7$ in mice indicated perfusion-mediated uptake of the radioligand in the brain followed by a relatively fast washout of activity. However, investigating the in vivo metabolism of $\left[{ }^{18} \mathrm{~F}\right] 7$ in mice disclosed the fast formation of several radiometabolites of which a considerable amount has been also detected in the brain. Moreover, in vitro autoradiographic studies in porcine brain revealed a high nonspecific binding of $\left[{ }^{18} \mathrm{~F}\right] 7$ which might be related to insufficient affinity toward PDE5.

In conclusion, the results of this study support the assumption that for PET imaging of the PDE5 enzyme, specific radioligands with an affinity in the sub-nanomolar range seem to be crucial.

\section{Experimental section}

\subsection{Organic chemistry}

\subsubsection{General}

All commercially available reagents and solvents were purchased from the following commercial suppliers: Sigma Aldrich, Acros Organics, Carlo Erba, and Alfa Aesar and were used without further purification. Room temperature (rt) refers to $20-25^{\circ} \mathrm{C}$. All solvents were dried using common techniques. Air and moisture sensitive reactions were carried out under anhydrous argon atmosphere. Magnesium sulfate $\left(\mathrm{MgSO}_{4}\right)$ and sodium sulfate $\left(\mathrm{Na}_{2} \mathrm{SO}_{4}\right)$ were used as drying agents. Monitoring of the reaction progress was performed using thin layer chromatography (TLC) on silica 60 F254 or alumina gel 60A + F254 plates and visualized with UV light (UV lamp Fisher Bioblock Scientific, $365 \mathrm{~nm}$ or $254 \mathrm{~nm}$ ) or with an appropriate staining agent. Column chromatography was performed on silica gel (Chromagel 60 ACC, 40-63 $\mu \mathrm{m}$, Carlo Erba Reagents) or on alumina gel (Merck, column chromatographic adsorption analysis ACC. to Brockmann, neutral aluminium oxide 90, standardised, 63-200 $\mu \mathrm{m}$ ). Uncorrected melting points ( $\mathrm{mp}$ ) were measured on an IA9100 Digital Melting Point Apparatus. Infrared spectra (IR) were recorded in the range of $4000-600 \mathrm{~cm}^{-1}$ on a Nicolet IS10 with attenuated total reflectance (ATR) accessory ( $v$ : stretch, as: asymmetric, s: symmetric, $\delta$ : deformation; op: out of plane). Nuclear magnetic resonance (NMR) spectra were recorded on a Bruker Avance 500 instrument $\left(500 \mathrm{MHz}\right.$ for ${ }^{1} \mathrm{H}, 125 \mathrm{MHz}$ for ${ }^{13} \mathrm{C}$ ), a Bruker Avance 400 instrument (400 MHz for ${ }^{1} \mathrm{H}, 100 \mathrm{MHz}$ for ${ }^{13} \mathrm{C}$ ) or a Bruker Avance 200 instrument (200 MHz for ${ }^{1} \mathrm{H}, 50 \mathrm{MHz}$ for ${ }^{13} \mathrm{C}$ ). All chemical shifts $(\delta)$ are reported in parts per million (ppm). Coupling constants $(J)$ are given in Hertz $(\mathrm{Hz})$. Spectral coupling patterns are indicated as follow: s: singlet; d: doublet; t: triplet; q: quartet; m: multiplet, brt: broad triplet and brs: broad singlet. All new compounds were analyzed by HRMS (High-Resolution Mass Spectrometry, Waters ${ }^{\circledR}$ Micromass ${ }^{\circledR}$ Q-Tof micro $^{\mathrm{TM}}$ 
Mass Spectrometer, UCA-START, Clermont-Auvergne University, Clermont-Ferrand, France). The isotope peaks for chlorine and bromine atoms are given with their relative intensities.

\subsubsection{Syntheses}

\subsubsection{Ethyl 8-bromo-6-cyano-4-[((6-fluoro-5-methoxypyridin-2- yl)methyl)amino]quinoline-3-carboxylate (12):}

$\mathrm{N}, \mathrm{N}$-Diisopropylethylamine (293 mg, $2.32 \mathrm{mmol}, 4.0$ eq.) and the crude product 10a $(550 \mathrm{mg}$, $2.85 \mathrm{mmol}, 5.0$ eq., synthesis in supporting information) were added under stirring to a solution of 11 [28] (193 mg, $0.57 \mathrm{mmol}, 1.0$ eq.) in $n$-propanol (15 mL). The resulting mixture was refluxed for 2 hours. After cooling to rt, water $(30 \mathrm{~mL})$ was added. The formed precipitate was collected by filtration, washed with ethanol $(10 \mathrm{~mL})$ and dried to give the desired product (160 mg, $0.35 \mathrm{mmol}, 61 \%)$ as a white solid. $\mathrm{R}_{\mathrm{f}}\left(\mathrm{SiO}_{2}\right.$, cyclohexane/ethyl acetate, $\left.7 / 3, \mathrm{v} / \mathrm{v}\right): 0.15$; Mp: $186 \pm 1{ }^{\circ} \mathrm{C} ; \mathrm{IR}\left(\mathrm{cm}^{-1}\right) \vee 2241\left(\mathrm{v}_{\mathrm{C} \equiv \mathrm{N}}\right), 1463\left(\delta_{\mathrm{CH}_{3}}\right), 1173\left(\mathrm{v}_{\mathrm{C}-\mathrm{o}}\right) ;{ }^{1} \mathrm{H}$ NMR (400 MHz, DMSO-d 6 ) $\delta 9.49$ (brs, $1 \mathrm{H}, \mathrm{NH}), 8.94\left(\mathrm{~m}, 2 \mathrm{H}, \mathrm{H}_{2}, \mathrm{H}_{5}\right), 8.49\left(\mathrm{~d}, 1 \mathrm{H},{ }^{3} \mathrm{~J}_{\mathrm{H} 7-\mathrm{H} 5}=1.5 \mathrm{~Hz}, \mathrm{H}_{7}\right), 7.65\left(\mathrm{dd}, 1 \mathrm{H},{ }^{3} \mathrm{H}_{\mathrm{H}^{\prime}-\mathrm{H} 6^{\prime}}\right.$ $\left.=8.1 \mathrm{~Hz},{ }^{4} J_{\mathrm{H} 5^{\prime}-\mathrm{F}}=10.5 \mathrm{~Hz}, \mathrm{H}_{5^{\prime}}\right), 7.32\left(\mathrm{~d}, 1 \mathrm{H},{ }^{3} \mathrm{JH}_{\mathrm{H}^{\prime}-\mathrm{H} 5^{\prime}}=8.1 \mathrm{~Hz}, \mathrm{H}_{6^{\prime}}\right), 4.92\left(\mathrm{~d}, 2 \mathrm{H},{ }^{3} \mathrm{~J}_{\mathrm{Hd}-\mathrm{NH}}=5.2 \mathrm{~Hz}\right.$, $\left.\mathrm{H}_{\mathrm{d}}\right), 4.30\left(\mathrm{q}, 2 \mathrm{H},{ }^{3} \mathrm{~J}_{\mathrm{Hb}-\mathrm{Hc}}=7.1 \mathrm{~Hz}, \mathrm{H}_{\mathrm{b}}\right), 3.86\left(\mathrm{~s}, 3 \mathrm{H}, \mathrm{H}_{\mathrm{e}}\right), 1.29\left(\mathrm{t}, 3 \mathrm{H},{ }^{3} \mathrm{~J}_{\mathrm{Hc}-\mathrm{Hb}}=7.1 \mathrm{~Hz}, \mathrm{H}_{\mathrm{c}}\right) ;{ }^{13} \mathrm{C} \mathrm{NMR}$

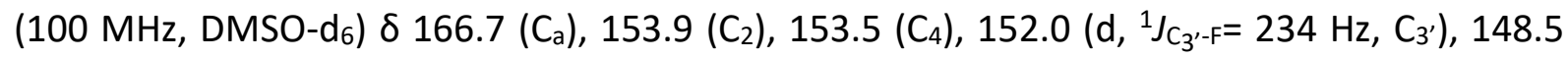
$\left(C_{8 \mathrm{a}}\right), 143.8\left(\mathrm{~d},{ }^{3} \mathrm{~J}_{\mathrm{C}^{\prime}-\mathrm{F}}=11 \mathrm{~Hz}, \mathrm{C}_{1^{\prime}}\right), 141.5\left(\mathrm{~d},{ }^{2} \mathrm{~J}_{\mathrm{C}^{\prime}{ }^{-\mathrm{F}}}=25 \mathrm{~Hz}, \mathrm{C}_{4^{\prime}}\right), 135.1\left(\mathrm{C}_{7}\right), 131.7\left(\mathrm{C}_{5}\right), 125.3\left(\mathrm{C}_{8}\right)$, $123.7\left(\mathrm{~d},{ }^{3} \int_{\mathrm{C}^{\prime}-\mathrm{F}}=3 \mathrm{~Hz}, \mathrm{C}_{5^{\prime}}\right), 120.9\left(\mathrm{~d},{ }^{4} \mathrm{~J}_{\mathrm{C}^{\prime}{ }^{\prime} \mathrm{F}}=4 \mathrm{~Hz}, \mathrm{C}_{6^{\prime}}\right), 119.8\left(\mathrm{C}_{4 \mathrm{a}}\right), 117.8(\mathrm{CN}), 107.3\left(\mathrm{C}_{6}\right), 105.4$ $\left(C_{3}\right), 61.2\left(C_{b}\right), 56.3\left(C_{e}\right), 50.4\left(C_{d}\right), 14.0\left(C_{c}\right)$; HRMS (ESI) $\mathrm{m} / z$ calculated for $\mathrm{C}_{20} \mathrm{H}_{17}{ }^{79} \mathrm{BrFN}_{4} \mathrm{O}_{3}$ $[\mathrm{M}+\mathrm{H}]^{+}: 459.0468$, found $\mathrm{C}_{20} \mathrm{H}_{17}{ }^{79} \mathrm{BrFN}_{4} \mathrm{O}_{3}[\mathrm{M}+\mathrm{H}]^{+}: 459.0474(100 \%) ; \mathrm{C}_{20} \mathrm{H}_{17}{ }^{81} \mathrm{BrFN}_{4} \mathrm{O}_{3}[\mathrm{M}+\mathrm{H}]^{+}$: 461.0457 (98\%).

\subsubsection{General procedure for $13 a$ and $13 b$ :}

Under stirring and argon atmosphere, cyclopropylboronic acid (3.0 eq.) or ethylboronic acid (16 eq.), tetrakis(triphenylphosphine)palladium(0) (15 mg, $12.9 \mu \mathrm{mol}, 0.06$ eq.) and caesium carbonate ( 2.5 eq.) were added to a solution of 12 (1.0 eq.) in anhydrous toluene $(10 \mathrm{~mL})$. The resulting mixture was refluxed until the TLC monitoring revealed the complete consumption of 12 (15 $\mathrm{h}$ for $13 \mathrm{a}$ and $22 \mathrm{~h}$ for $\mathbf{1 3 b}$ ). After cooling to room temperature, the solvent was evaporated under reduced pressure. The crude product was purified by column chromatography to give $13 \mathbf{a}$ or $13 \mathbf{b}$ as white solid.

\subsection{Ethyl 6-cyano-8-ethyl-4-[((6-fluoro-5-methoxypyridin-2- yl)methyl)amino]quinoline-3-carboxylate (13a):}

Column chromatography conditions: $\mathrm{SiO}_{2}$, ethyl acetate/cyclohexane, 5/5, v/v; yield: $68 \%$ (60 $\mathrm{mg}, 0.15 \mathrm{mmol}) ; \mathrm{R}_{\mathrm{f}}\left(\mathrm{SiO}_{2}\right.$, cyclohexane/ethyl acetate, 6/4, v/v): 0.65; $\mathrm{Mp}: 152 \pm 1{ }^{\circ} \mathrm{C} ; \mathrm{IR}\left(\mathrm{cm}^{-1}\right)$ v $2229\left(v_{\mathrm{C}=\mathrm{N}}\right), 1687\left(\mathrm{v}_{\mathrm{C}=\mathrm{O}}\right), 1570\left(\delta_{\mathrm{N}-\mathrm{H}}\right), 1358\left(\delta_{\mathrm{CH} 3}\right), 1283\left(\mathrm{v}_{\mathrm{C}-\mathrm{O}}\right), 1187\left(\mathrm{v}_{\mathrm{C}-\mathrm{O}}\right) ;{ }^{1} \mathrm{H}$ NMR $(400 \mathrm{MHz}$, $\left.\mathrm{CDCl}_{3}\right) \delta 9.80$ (brs, $\left.1 \mathrm{H}, \mathrm{NH}\right), 9.21\left(\mathrm{~s}, 1 \mathrm{H}, \mathrm{H}_{2}\right), 8.39\left(\mathrm{~d}, 1 \mathrm{H},{ }^{4} \mathrm{~J}_{\mathrm{H} 5-\mathrm{H} 7}=1.7 \mathrm{~Hz}, \mathrm{H}_{5}\right), 7.64\left(\mathrm{~d}, 1 \mathrm{H},{ }^{4} \mathrm{~J}_{\mathrm{H} 7-\mathrm{H} 5}\right.$ $\left.=1.7 \mathrm{~Hz}, \mathrm{H}_{7}\right), 7.31\left(\mathrm{dd}, 1 \mathrm{H},{ }^{3} \mathrm{~J}_{\mathrm{H} 5^{\prime}-\mathrm{H} 6^{\prime}}=8.0 \mathrm{~Hz},{ }^{3} \mathrm{~J}_{\mathrm{H} 5^{\prime}-\mathrm{HF}}=9.8 \mathrm{~Hz}, \mathrm{H}_{5^{\prime}}\right), 7.23\left(\mathrm{~d}, 1 \mathrm{H},{ }^{3} \mathrm{~J}_{\mathrm{H}^{\prime}-\mathrm{H} 5^{\prime}}=8.0 \mathrm{~Hz}\right.$, $\left.\mathrm{H}_{6^{\prime}}\right), 4.92\left(\mathrm{~d}, 2 \mathrm{H},{ }^{3} \mathrm{~J}_{\mathrm{Hd}-\mathrm{NH}}=5.5 \mathrm{~Hz}, \mathrm{H}_{\mathrm{d}}\right), 4.39\left(\mathrm{q}, 2 \mathrm{H},{ }^{3} \mathrm{~J}_{\mathrm{Hb}-\mathrm{Hc}}=7.1 \mathrm{~Hz}, \mathrm{H}_{\mathrm{b}}\right), 3.91\left(\mathrm{~s}, 3 \mathrm{H}, \mathrm{H}_{\mathrm{e}}\right), 3.19$ (q, 
$\left.2 \mathrm{H},{ }^{3} \mathrm{~J}_{\mathrm{Hf}-\mathrm{Hg}}=7.5 \mathrm{~Hz}, \mathrm{H}_{\mathrm{f}}\right), 1.40\left(\mathrm{t}, 3 \mathrm{H},{ }^{3} \mathrm{~J}_{\mathrm{Hc}-\mathrm{Hb}}=7.1 \mathrm{~Hz}, \mathrm{H}_{\mathrm{c}}\right), 1.32\left(\mathrm{t}, 3 \mathrm{H},{ }^{3} \mathrm{~J}_{\mathrm{Hg}-\mathrm{Hf}}=7.5 \mathrm{~Hz}, \mathrm{Hg}_{\mathrm{g}}\right) ;{ }^{13} \mathrm{C} \mathrm{NMR}$ $\left(100 \mathrm{MHz}, \mathrm{CDCl}_{3}\right) \delta 168.0\left(\mathrm{C}_{\mathrm{a}}\right), 156.6\left(\mathrm{C}_{4}\right), 154.1\left(\mathrm{~d},{ }^{1} \mathrm{~J}_{\mathrm{C}^{\prime}-\mathrm{F}}=241 \mathrm{~Hz}, \mathrm{C}_{3^{\prime}}\right), 152.6\left(\mathrm{C}_{2}\right), 151.0\left(\mathrm{C}_{8 \mathrm{a}}\right)$, $144.9\left(C_{8}\right), 144.1\left(d,{ }^{3} J_{C^{\prime}-F}=11 \mathrm{~Hz}, C_{1^{\prime}}\right), 142.4\left(d,{ }^{2} J_{C^{\prime}-F}=25 \mathrm{~Hz}, C_{4^{\prime}}\right), 130.1\left(C_{7}\right), 129.7\left(C_{5}\right), 122.3$ $\left(d,{ }^{3} J_{C 5^{\prime}-F}=4 \mathrm{~Hz}, C_{5^{\prime}}\right), 119.4\left(d,{ }^{4} J_{C^{\prime}-F}=4 \mathrm{~Hz}, C_{6^{\prime}}\right), 119.1(C N), 118.7\left(C_{4 a}\right), 107.0\left(C_{6}\right), 104.8\left(C_{3}\right)$, $61.1\left(C_{b}\right), 56.3\left(C_{e}\right), 52.5\left(C_{d}\right), 24.9\left(C_{f}\right), 14.3\left(C_{g}\right), 14.1\left(C_{c}\right)$; HRMS (ESI) $m / z$ calculated for $\mathrm{C}_{22} \mathrm{H}_{22} \mathrm{FN}_{4} \mathrm{O}_{3}[\mathrm{M}+\mathrm{H}]^{+}:$409.1676, found 409.1657 .

\subsection{Ethyl 6-cyano-8-cyclopropyl-4-[((6-fluoro-5-methoxypyridin-2-yl)methyl)amino] quinoline-3-carboxylate (13b):}

Column chromatography conditions: $\mathrm{SiO}_{2}$, ethyl acetate/cyclohexane, 4/6, v/v; yield: $86 \%$ (80 $\mathrm{mg}, 0.19 \mathrm{mmol}) ; \mathrm{R}_{\mathrm{f}}\left(\mathrm{Al}_{2} \mathrm{O}_{3}\right.$, cyclohexane/ ethyl acetate, 5/5, v/v): 0.74; $\mathrm{Mp}: 155 \pm 1{ }^{\circ} \mathrm{C} ; \mathrm{IR}\left(\mathrm{cm}^{-}\right.$ $\left.{ }^{1}\right)$ v $2226\left(\mathrm{v}_{\mathrm{C}=\mathrm{N}}\right), 1667\left(\mathrm{v}_{\mathrm{C}=\mathrm{O}}\right), 1473\left(\delta_{\mathrm{CH} 3}\right), 1198\left(\mathrm{v}_{\mathrm{C}-\mathrm{O}}\right) ;{ }^{1} \mathrm{H} \mathrm{NMR}\left(400 \mathrm{MHz}, \mathrm{CDCl}_{3}\right) \delta 9.98(\mathrm{brs}, 1 \mathrm{H}$, $\mathrm{NH}), 9.27\left(\mathrm{~s}, 1 \mathrm{H}, \mathrm{H}_{2}\right), 8.36\left(\mathrm{~d}, 1 \mathrm{H},{ }^{4} J_{\mathrm{H} 5-\mathrm{H} 7}=1.4 \mathrm{~Hz}, \mathrm{H}_{5}\right), 7.29\left(\mathrm{~m}, 2 \mathrm{H}, \mathrm{H}_{7}, \mathrm{H}_{5^{\prime}}\right), 7.22\left(\mathrm{~d}, 1 \mathrm{H},{ }^{3} \mathrm{JH}_{\mathrm{H}^{\prime}-\mathrm{H} 5^{\prime}}\right.$ $\left.=8.1 \mathrm{~Hz}, \mathrm{H}_{6^{\prime}}\right), 4.93\left(\mathrm{~d}, 2 \mathrm{H},{ }^{3} \mathrm{~J}_{\mathrm{Hd}-\mathrm{NH}}=5.5 \mathrm{~Hz}, \mathrm{H}_{\mathrm{d}}\right), 4.40\left(\mathrm{q}, 2 \mathrm{H},{ }^{3} \mathrm{~J}_{\mathrm{Hb}-\mathrm{Hc}}=7.1 \mathrm{~Hz}, \mathrm{H}_{\mathrm{b}}\right), 3.91\left(\mathrm{~s}, 3 \mathrm{H}, \mathrm{H}_{\mathrm{e}}\right)$, $3.04\left(\mathrm{~m}, 1 \mathrm{H}, \mathrm{H}_{\mathrm{f}}\right), 1.39\left(\mathrm{t}, 3 \mathrm{H},{ }^{3} \mathrm{~J} \mathrm{Hc}-\mathrm{Hb}=7.1 \mathrm{~Hz}, \mathrm{H}_{\mathrm{b}}\right), 1.23\left(\mathrm{~m}, 2 \mathrm{H}, \mathrm{H}_{\mathrm{g}}\right.$ or $\left.\mathrm{H}_{\mathrm{g}^{\prime}}\right), 0.79\left(\mathrm{~m}, 2 \mathrm{H}, \mathrm{H}_{\mathrm{g}}\right.$ or $\left.\mathrm{H}_{\mathrm{g}^{\prime}}\right)$; ${ }^{13} \mathrm{C} \mathrm{NMR}\left(100 \mathrm{MHz}, \mathrm{CDCl}_{3}\right) \delta 167.7\left(\mathrm{C}_{\mathrm{a}}\right), 156.8\left(\mathrm{C}_{4}\right), 153.1\left(\mathrm{~d},{ }^{1} \mathrm{~J}_{\mathrm{C}^{\prime}-\mathrm{F}}=241 \mathrm{~Hz}, \mathrm{C}_{3^{\prime}}\right), 152.1\left(\mathrm{C}_{2}\right)$, $144.3\left(\mathrm{C}_{8 \mathrm{a}}\right), 143.7\left(2 \mathrm{C}, \mathrm{C}_{8}, \mathrm{C}_{1^{\prime}}\right), 142.5\left(\mathrm{~d},{ }^{2} \mathrm{~J}_{\mathrm{C}^{\prime}-\mathrm{F}}=25 \mathrm{~Hz}, \mathrm{C}_{4^{\prime}}\right), 128.8\left(\mathrm{C}_{5}\right), 126.4\left(\mathrm{C}_{7}\right), 122.3\left(\mathrm{~d},{ }^{3} \mathrm{~J}_{\mathrm{C}^{\prime}-}\right.$ $\left.\mathrm{F}=4 \mathrm{~Hz}, \mathrm{C}_{5^{\prime}}\right), 119.4\left(\mathrm{~d},{ }^{3} \mathrm{~J}_{\mathrm{C}^{\prime}-\mathrm{F}}=4 \mathrm{~Hz}, \mathrm{C}_{6^{\prime}}\right), 118.9\left(\mathrm{CN}\right.$ or $\left.\mathrm{C}_{4 \mathrm{a}}\right), 118.5\left(\mathrm{CN}\right.$ or $\left.\mathrm{C}_{4 \mathrm{a}}\right), 107.4\left(\mathrm{C}_{6}\right), 104.8$ $\left(C_{3}\right), 61.3\left(C_{b}\right), 56.3\left(C_{e}\right), 52.4\left(C_{d}\right), 14.1\left(C_{c}\right), 11.3\left(C_{f}\right), 9.7\left(2 C, C_{g}, C_{g^{\prime}}\right)$; HRMS (ESI) m/z calculated for $\mathrm{C}_{23} \mathrm{H}_{22} \mathrm{FN}_{4} \mathrm{O}_{3}[\mathrm{M}+\mathrm{H}]^{+}:$421.1676, found 421.1688 .

\subsubsection{General procedure for 3 and 4 :}

A 1.1 M solution of lithium tri-tert-butoxyaluminum hydride in anhydrous tetrahydrofuran (2.7 $\mathrm{mL}, 2.97 \mathrm{mmol}, 6.0$ eq.) was added to a solution of 13a or 13b (1.0 eq.) in anhydrous tetrahydrofuran $(15 \mathrm{~mL})$ under stirring and argon atmosphere. The resulting mixture was heated at $50{ }^{\circ} \mathrm{C}$ for 30 hours. After cooling to room temperature, an $0.1 \mathrm{M}$ aqueous sodium hydroxide solution ( $40 \mathrm{~mL}$ ) was slowly added under stirring $(\mathrm{pH} 10-11)$. The aqueous layer was extracted with ethyl acetate $(4 \times 40 \mathrm{~mL})$. The combined organic layers were washed with brine $(60 \mathrm{~mL})$, dried over anhydrous magnesium sulfate, filtered and then concentrated under reduced pressure. The crude product was purified by chromatography $\left(\mathrm{SiO}_{2}\right.$, ethanol/dichloromethane, 1/9, v/v) to give the desired products 3 (white solid) or $\mathbf{4}$ (light yellow solid).

\subsection{8-Ethyl-4-[((6-fluoro-5-methoxypyridin-2-yl)methyl)amino]-3- (hydroxymethyl)quinoline-6-carbonitrile (3):}

Yield: 36\% (65 mg, $0.18 \mathrm{mmol}) ; \mathrm{R}_{\mathrm{f}}\left(\mathrm{SiO}_{2}\right.$, ethanol/dichloromethane, 1/9, v/v): 0.29; Mp: $168 \pm$ $1^{\circ} \mathrm{C}$; IR $\left(\mathrm{cm}^{-1}\right) \vee 3420\left(\mathrm{v}_{\mathrm{N}-\mathrm{H}}\right), 2228\left(\mathrm{v}_{\mathrm{C} \equiv \mathrm{N}}\right), 1563\left(\delta_{\mathrm{N}-\mathrm{H}}\right), 1479\left(\delta_{\mathrm{CH} 3}\right), 1010\left(\mathrm{v}_{\mathrm{C}-\mathrm{O}}\right) ;{ }^{1} \mathrm{H}$ NMR $(400 \mathrm{MHz}$, DMSO-d 6 ) $\delta 8.81\left(\mathrm{~d}, 1 \mathrm{H},{ }^{4} J_{\mathrm{H} 5-\mathrm{H} 7}=1.5 \mathrm{~Hz}, \mathrm{H}_{5}\right), 8.48\left(\mathrm{~s}, 1 \mathrm{H}, \mathrm{H}_{2}\right), 7.75\left(\mathrm{~d}, 1 \mathrm{H},{ }^{4} J_{\mathrm{H} 7-\mathrm{H} 5}=1.5 \mathrm{~Hz}, \mathrm{H}_{7}\right)$, $7.59\left(\mathrm{dd}, 1 \mathrm{H},{ }^{3} J_{\mathrm{H} 5^{\prime}-\mathrm{H} 6^{\prime}}=8.1 \mathrm{~Hz},{ }^{4} J_{\mathrm{H} 5^{\prime}-\mathrm{F}}=10.6 \mathrm{~Hz}, \mathrm{H}_{5^{\prime}}\right), 7.53\left(\mathrm{t}, 1 \mathrm{H},{ }^{3} J_{\mathrm{NH}-\mathrm{Hb}}=6.7 \mathrm{~Hz}, \mathrm{NH}\right), 7.21(\mathrm{~d}$, $\left.1 \mathrm{H},{ }^{3} \mathrm{~J}_{\mathrm{H} 6^{\prime}-\mathrm{H} 5^{\prime}}=8.1 \mathrm{~Hz}, \mathrm{H}_{6^{\prime}}\right), 5.36\left(\mathrm{t}, 1 \mathrm{H},{ }^{3} \mathrm{~J}_{\mathrm{OH}-\mathrm{Ha}}=5.1 \mathrm{~Hz}, \mathrm{OH}\right), 4.84\left(\mathrm{~d}, 2 \mathrm{H},{ }^{3} \mathrm{~J}_{\mathrm{Hb}-\mathrm{NH}}=6.7 \mathrm{~Hz}, \mathrm{Hb}_{\mathrm{b}}\right), 4.47$ $\left(\mathrm{d}, 2 \mathrm{H}, 3^{3} \mathrm{Ha}_{\mathrm{HaH}}=5.1 \mathrm{~Hz}, \mathrm{H}_{\mathrm{a}}\right), 3.83\left(\mathrm{~s}, 3 \mathrm{H}, \mathrm{H}_{\mathrm{c}}\right), 3.13\left(\mathrm{q}, 2 \mathrm{H},{ }^{3} \mathrm{~J}_{\mathrm{Hd}-\mathrm{He}}=7.5 \mathrm{~Hz}, \mathrm{H}_{\mathrm{d}}\right), 1.25\left(\mathrm{t}, 3 \mathrm{H}, 3^{3} \mathrm{He}_{\mathrm{Hd}}\right.$ $\left.=7.5 \mathrm{~Hz}, \mathrm{H}_{\mathrm{e}}\right) ;{ }^{13} \mathrm{C}$ NMR (100 MHz, DMSO-d $\left.\mathrm{d}_{6}\right) \delta 155.2\left(\mathrm{C}_{2}\right), 151.9\left(\mathrm{~d},{ }^{1} \mathrm{~J}_{\mathrm{C}^{\prime}-\mathrm{F}}=236 \mathrm{~Hz}, \mathrm{C}_{3^{\prime}}\right), 149.9$ $\left(C_{4}\right), 148.1\left(C_{8 a}\right), 147.5\left(d,{ }^{3} J_{C 1^{\prime}-F}=11 \mathrm{~Hz}, C_{1^{\prime}}\right), 144.1\left(C_{8}\right), 140.9\left(d,{ }^{2} J_{C 4^{\prime}-F}=25 \mathrm{~Hz}, C_{4^{\prime}}\right), 127.6\left(C_{7}\right)$, 
$127.4\left(C_{5}\right), 123.6\left(d,{ }^{3} J_{C^{\prime}-F}=4 \mathrm{~Hz}, C_{5^{\prime}}\right), 119.6\left(2 \mathrm{C}, \mathrm{CN}, \mathrm{C}_{4 \mathrm{a}}\right), 119.2\left(\mathrm{~d},{ }^{4} \mathrm{~J}_{\mathrm{C}^{\prime}-\mathrm{F}}=4 \mathrm{~Hz}, \mathrm{C}_{6^{\prime}}\right), 115.5\left(\mathrm{C}_{3}\right)$, $106.1\left(C_{6}\right), 59.7\left(C_{a}\right), 56.2\left(C_{c}\right), 48.8\left(C_{b}\right), 24.2\left(C_{d}\right), 14.8\left(C_{e}\right)$; HRMS (ESI) $m / z$ calculated for $\mathrm{C}_{20} \mathrm{H}_{20} \mathrm{FN}_{4} \mathrm{O}_{2}[\mathrm{M}+\mathrm{H}]^{+}:$367.1570; found 367.1528.

\subsection{8-Cyclopropyl-4-[((6-fluoro-5-methoxypyridin-2-yl)methyl)amino]-3- (hydroxymethyl)quinoline-6-carbonitrile (4):}

Yield: 45\% (120 mg, $0.32 \mathrm{mmol}) ; \mathrm{R}_{\mathrm{f}}\left(\mathrm{SiO}_{2}\right.$, ethanol/dichloromethane, 1/9, v/v): 0.40; Mp: 183 $\pm 1{ }^{\circ} \mathrm{C} ; \mathrm{IR}\left(\mathrm{cm}^{-1}\right)$ v $2226\left(\mathrm{v}_{\mathrm{C} \equiv \mathrm{N}}\right), 1542\left(\delta_{\mathrm{N}-\mathrm{H}}\right), 1478\left(\delta_{\mathrm{CH} 3}\right), 1260\left(\mathrm{v}_{\mathrm{C}-\mathrm{O}}\right), 1036\left(\mathrm{v}_{\mathrm{C}-\mathrm{O}}\right) ;{ }^{1} \mathrm{H}$ NMR (400 $\left.\mathrm{MHz}, \mathrm{DMSO}-\mathrm{d}_{6}\right) \delta 8.73\left(\mathrm{~d}, 1 \mathrm{H},{ }^{4} \mathrm{~J}_{\mathrm{H} 5-\mathrm{H} 7}=1.4 \mathrm{~Hz}, \mathrm{H}_{5}\right), 8.51\left(\mathrm{~s}, 1 \mathrm{H}, \mathrm{H}_{2}\right), 7.58\left(\mathrm{dd}, 1 \mathrm{H},{ }^{3} \mathrm{JH}_{\mathrm{H}^{\prime}-\mathrm{H}^{\prime}}=8.2\right.$ $\left.\mathrm{Hz},{ }^{4} J_{\mathrm{H}^{\prime}-\mathrm{F}}=10.5 \mathrm{~Hz}, \mathrm{H}_{5^{\prime}}\right), 7.50\left(\mathrm{t}, 1 \mathrm{H},{ }^{3} J_{\mathrm{NH}-\mathrm{Hb}}=6.6 \mathrm{~Hz}, \mathrm{NH}\right), 7.35\left(\mathrm{~d}, 1 \mathrm{H},{ }^{4} J_{\mathrm{H} 7-\mathrm{H} 5}=1.4 \mathrm{~Hz}, \mathrm{H}_{7}\right), 7.21$ $\left(\mathrm{d}, 1 \mathrm{H},{ }^{3} \mathrm{H}_{\mathrm{H}^{\prime}-\mathrm{H} 5^{\prime}}=8.2 \mathrm{~Hz}, \mathrm{H}_{6^{\prime}}\right), 5.39\left(\mathrm{t}, 1 \mathrm{H},{ }^{3} \mathrm{~J}_{\mathrm{OH}-\mathrm{Ha}}=5.1 \mathrm{~Hz}, \mathrm{OH}\right), 4.84\left(\mathrm{~d}, 2 \mathrm{H},{ }^{3} \mathrm{~J}_{\mathrm{Hb}-\mathrm{NH}}=6.6 \mathrm{~Hz}, \mathrm{H}_{\mathrm{b}}\right)$, $4.49\left(\mathrm{~d}, 2 \mathrm{H}, 3^{3} \mathrm{Ha}_{\mathrm{HaH}}=5.1 \mathrm{~Hz}, \mathrm{H}_{\mathrm{a}}\right), 3.83\left(\mathrm{~s}, 3 \mathrm{H}, \mathrm{H}_{\mathrm{c}}\right), 3.13\left(\mathrm{~m}, 1 \mathrm{H}, \mathrm{H}_{\mathrm{d}}\right), 1.11\left(\mathrm{~m}, 2 \mathrm{H}, \mathrm{H}_{\mathrm{e}}\right.$ or $\left.\mathrm{H}_{\mathrm{e}^{\prime}}\right), 0.85$ $\left(\mathrm{m}, 2 \mathrm{H}, \mathrm{H}_{\mathrm{e}}\right.$ or $\left.\mathrm{H}_{\mathrm{e}^{\prime}}\right) ;{ }^{13} \mathrm{C} \mathrm{NMR}\left(100 \mathrm{MHz}, \mathrm{DMSO}-\mathrm{d}_{6}\right) \delta 155.1\left(\mathrm{C}_{2}\right), 151.9\left(\mathrm{~d},{ }^{1} \mathrm{~J}_{\mathrm{C} 3^{\prime}-\mathrm{F}}=236 \mathrm{~Hz}, \mathrm{C}_{3^{\prime}}\right)$, $149.9\left(C_{4}\right), 148.7\left(C_{8 a}\right), 147.5\left(d,{ }^{3} J_{C 1^{\prime}-F}=11 \mathrm{~Hz}, C_{1^{\prime}}\right), 144.4\left(C_{8}\right), 140.9\left(d,{ }^{2} J_{C 4^{\prime}-F}=25 \mathrm{~Hz}, C_{4^{\prime}}\right), 126.2$ $\left(C_{5}\right), 123.5\left(d,{ }^{3} J_{C 5^{\prime}-F}=4 \mathrm{~Hz}, C_{5^{\prime}}\right), 122.5\left(C_{7}\right), 119.6(C N), 119.5\left(C_{3}\right), 119.2\left(d,{ }^{4} J_{C^{\prime}-F}=3 \mathrm{~Hz}, C_{6^{\prime}}\right)$, $115.7\left(C_{4 a}\right), 106.4\left(C_{6}\right), 59.7\left(C_{a}\right), 56.1\left(C_{c}\right), 48.9\left(C_{b}\right), 10.6\left(C_{d}\right), 10.1\left(2 C, C_{e}, C_{e^{\prime}}\right) ; H R M S(E S I) m / z$ calculated for $\mathrm{C}_{21} \mathrm{H}_{20} \mathrm{FN}_{4} \mathrm{O}_{2}[\mathrm{M}+\mathrm{H}]^{+}$: 379.1570, found 379.1535 .

\subsubsection{Ethyl 4-[(3-chloro-4-methoxybenzyl)amino]-6-cyano-8-(3-hydroxypropyl)quinoline- 3-carboxylate (15):}

A solution of 0.5 M 9-borabicyclo[3.3.1]nonane (9-BBN) in anhydrous tetrahydrofuran ( $25 \mathrm{~mL}$, $12.5 \mathrm{mmol}, 6.0$ eq.) was slowly added at $0{ }^{\circ} \mathrm{C}$ and under argon atmosphere to a solution of allyl alcohol $(0.86 \mathrm{~mL}, 12.6 \mathrm{mmol}, 6.0$ eq.) in anhydrous and previously degassed tetrahydrofuran $(3.0 \mathrm{~mL})$. The resulting mixture was stirred at $\mathrm{rt}$ for 7 hours. This solution was then added, under argon atmosphere, to a mixture of 14 [28] (1.0 g, $2.11 \mathrm{mmol}, 1.0$ eq.), tetrakis(triphenylphosphine)palladium(0) $(0.24 \mathrm{~g}, 0.21 \mathrm{mmol}, 0.1 \mathrm{eq}$.$) , potassium carbonate$ $(1.16 \mathrm{~g}, 8.39 \mathrm{mmol}, 4.0$ eq. $)$, and water $(1.0 \mathrm{~mL})$ in anhydrous $\mathrm{N}, \mathrm{N}$-dimethylformamide $(40 \mathrm{~mL})$. The resulting mixture was stirred at $80^{\circ} \mathrm{C}$ for 22 hours. After cooling to room temperature, water $(40 \mathrm{~mL})$ was added and the aqueous layer was extracted with ethyl acetate $(3 \times 50 \mathrm{~mL})$. The combined organic layers were washed with brine $(3 \times 100 \mathrm{~mL})$, dried with anhydrous magnesium sulfate, filtered and concentrated under reduced pressure. The crude residue was purified by column chromatography $\left(\mathrm{SiO}_{2}\right.$, ethyl acetate/cyclohexane, 5/5, v/v) to give by order of elution the desired product $15(0.25 \mathrm{~g}, 0.55 \mathrm{mmol}, 26 \%)$ as a yellow solid, and ethyl 4-[(3chloro-4-methoxybenzyl)amino]-6-cyanoquinoline-3-carboxylate $(0.24 \mathrm{~g}, 0.61 \mathrm{mmol}, 29 \%)$. $\mathrm{R}_{\mathrm{f}}$ $\left(\mathrm{SiO}_{2}\right.$, cyclohexane/ethyl acetate, 5/5, v/v): 0.17; Mp: $118 \pm 1^{\circ} \mathrm{C} ; \mathrm{IR}\left(\mathrm{cm}^{-1}\right) \vee 2231\left(\mathrm{v}_{\mathrm{C} \equiv \mathrm{N}}\right), 1673$ $\left(v_{\mathrm{C}=0}\right), 1595\left(v_{\mathrm{C}=\mathrm{C}}\right), 1505\left(\delta_{\mathrm{N}-\mathrm{H}}\right), 1262\left(\mathrm{v}_{\mathrm{C}-\mathrm{O}}\right), 1190\left(\mathrm{v}_{\mathrm{c}-\mathrm{o}}\right), 1068\left(\mathrm{v}_{\mathrm{C}-\mathrm{O}}\right) ;{ }^{1} \mathrm{H} \mathrm{NMR}\left(400 \mathrm{MHz}, \mathrm{CDCl}_{3}\right) \delta$ 9.80 (brt, $1 \mathrm{H}, \mathrm{NH}), 9.18\left(\mathrm{~s}, 1 \mathrm{H}, \mathrm{H}_{2}\right), 8.40\left(\mathrm{~d}, 1 \mathrm{H},{ }^{4} J_{\mathrm{H} 5-\mathrm{H} 7}=1.7 \mathrm{~Hz}, \mathrm{H}_{5}\right), 7.70\left(\mathrm{~d},{ }^{4} \mathrm{~J}_{\mathrm{H} 7-\mathrm{H} 5}=1.7 \mathrm{~Hz}, \mathrm{H}_{7}\right)$, $7.39\left(\mathrm{~d}, 1 \mathrm{H},{ }^{4} \mathrm{~J}_{\mathrm{H} 2^{\prime}-\mathrm{H} 6^{\prime}}=2.2 \mathrm{~Hz}, \mathrm{H}_{2^{\prime}}\right), 7.26\left(\mathrm{dd}, 1 \mathrm{H},{ }^{3} J_{\mathrm{H} 6^{\prime}-\mathrm{H} 5^{\prime}}=8.4 \mathrm{~Hz},{ }^{4} J_{\mathrm{H}^{\prime}-\mathrm{H} 2^{\prime}}=2.2 \mathrm{~Hz}, \mathrm{H}_{6^{\prime}}\right), 6.97(\mathrm{~d}$, $\left.1 \mathrm{H},{ }^{3} J_{\mathrm{H} 5^{\prime}-\mathrm{H} 6^{\prime}}=8.4 \mathrm{~Hz}, \mathrm{H}_{5^{\prime}}\right), 4.88\left(\mathrm{~d}, 2 \mathrm{H},{ }^{3} \mathrm{~J}_{\mathrm{Hd}-\mathrm{NH}}=5.5 \mathrm{~Hz}, \mathrm{H}_{\mathrm{d}}\right), 4.36\left(\mathrm{q}, 2 \mathrm{H},{ }^{3} J_{\mathrm{Hb}-\mathrm{Hc}}=7.1 \mathrm{~Hz}, \mathrm{H}_{\mathrm{b}}\right), 3.91$ $\left(\mathrm{s}, 3 \mathrm{H}, \mathrm{H}_{\mathrm{e}}\right), 3.39\left(\mathrm{~m}, 2 \mathrm{H}, \mathrm{H}_{\mathrm{h}}\right), 3.29\left(\mathrm{~m}, 2 \mathrm{H}, \mathrm{H}_{\mathrm{f}}\right), 1.94\left(\mathrm{~m}, 2 \mathrm{H}, \mathrm{H}_{\mathrm{g}}\right), 1.40\left(\mathrm{t}, 3 \mathrm{H},{ }^{3} \mathrm{~J} \mathrm{Hc}-\mathrm{Hb}=7.1 \mathrm{~Hz}, \mathrm{H}_{\mathrm{c}}\right)$; ${ }^{13} \mathrm{C} \mathrm{NMR}\left(100 \mathrm{MHz}, \mathrm{CDCl}_{3}\right) \delta 168.2\left(\mathrm{C}_{\mathrm{a}}\right), 156.8\left(\mathrm{C}_{4}\right), 155.1\left(\mathrm{C}_{4^{\prime}}\right), 153.0\left(\mathrm{C}_{2}\right), 151.5\left(\mathrm{C}_{8 \mathrm{a}}\right), 142.8$ $\left(C_{8}\right), 132.7\left(C_{7}\right), 130.5\left(C_{5}\right), 130.2\left(C_{1^{\prime}}\right), 129.4\left(C_{2^{\prime}}\right), 126.9\left(C_{6^{\prime}}\right), 123.5\left(C_{3^{\prime}}\right), 118.8(C N), 118.6$ 
( $\left.C_{4 a}\right), 112.6\left(C_{5^{\prime}}\right), 107.4\left(C_{6}\right), 104.3\left(C_{3}\right), 61.5\left(C_{b}\right), 59.5\left(C_{h}\right), 56.3\left(C_{e}\right), 52.0\left(C_{d}\right), 33.9\left(C_{g}\right), 27.0$

$\left(\mathrm{C}_{f}\right), 14.3\left(\mathrm{C}_{\mathrm{c}}\right)$; HRMS (ESI) $\mathrm{m} / \mathrm{z}$ calculated for $\mathrm{C}_{24} \mathrm{H}_{25}{ }^{35} \mathrm{CIN}_{3} \mathrm{O}_{4}[\mathrm{M}+\mathrm{H}]^{+}:$454.1534, found $\mathrm{C}_{24} \mathrm{H}_{25}{ }^{35} \mathrm{CIN}_{3} \mathrm{O}_{4}[\mathrm{M}+\mathrm{H}]^{+}: 454.1559$ (100\%); $\mathrm{C}_{24} \mathrm{H}_{25}{ }^{37} \mathrm{CIN}_{3} \mathrm{O}_{4}[\mathrm{M}+\mathrm{H}]^{+}: 456.1554$ (33\%).

\subsubsection{Ethyl 1-[(3-chloro-4-methoxybenzyl)imino]-9-cyano-6,7-dihydro- $1 \mathrm{H}, 5 \mathrm{H}$ - pyrido[3,2,1-ij]quinoline-2-carboxylate (17):}

Diethylaminosulfur trifluoride (DAST, $41 \mu \mathrm{L}, 0.31 \mathrm{mmol}, 2.0$ eq.) was added dropwise, at $-60^{\circ} \mathrm{C}$ and under stirring, to a solution of 15 (70 $\mathrm{mg}, 0.15 \mathrm{mmol}, 1.0$ eq.) in anhydrous dichloromethane $(5.0 \mathrm{~mL})$. The temperature was slowly increased to $-30^{\circ} \mathrm{C}$ and the mixture was stirred between $-30{ }^{\circ} \mathrm{C}$ and $-20^{\circ} \mathrm{C}$ for 2 hours before addition of an aqueous saturated sodium bicarbonate solution $(10 \mathrm{~mL})$ at $-20^{\circ} \mathrm{C}(\mathrm{pH}=7-8)$. This mixture was extracted with dichloromethane $(2 \times 15 \mathrm{~mL})$. The combined organic layers were washed with brine $(50 \mathrm{~mL})$, dried over anhydrous magnesium sulfate, filtered and evaporated under reduced pressure. The residue was purified by column chromatography $\left(\mathrm{Al}_{2} \mathrm{O}_{3}\right.$, ethyl acetate/cyclohexane, $7 / 3$, $\mathrm{v} / \mathrm{v}$ ) to give 17 (30 mg, $84.8 \mu \mathrm{mol}, 57 \%)$ as a yellow solid. $\mathrm{R}_{\mathrm{f}}\left(\mathrm{SiO}_{2}\right.$, cyclohexane/ethyl acetate, 3/7, v/v): 0.25; Mp: $152 \pm 1{ }^{\circ} \mathrm{C} ; \mathrm{IR}\left(\mathrm{cm}^{-1}\right)$ v $2224\left(\mathrm{v}_{\mathrm{C} \equiv \mathrm{N}}\right), 1702\left(\mathrm{v}_{\mathrm{C}=\mathrm{O}}\right), 1574\left(\mathrm{v}_{\mathrm{C}=\mathrm{N}}\right), 1502\left(\mathrm{v}_{\mathrm{C}=\mathrm{C}}\right), 1271$ (vc-o), 1170 (vc-o), 1062 (vc-o); ${ }^{1} \mathrm{H}$ NMR (400 MHz, CDCl $) \delta 8.53\left(\mathrm{~s}, 1 \mathrm{H}, \mathrm{H}_{5}\right), 7.73\left(\mathrm{~s}, 1 \mathrm{H}, \mathrm{H}_{2}\right), 7.38$

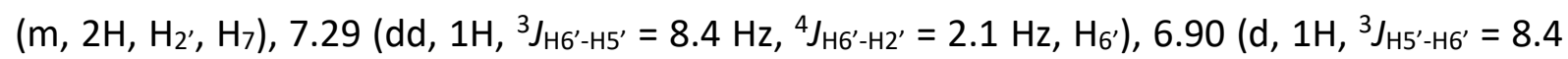
$\left.\mathrm{Hz}, \mathrm{H}_{5^{\prime}}\right), 4.33\left(\mathrm{~s}, 2 \mathrm{H}, \mathrm{H}_{\mathrm{d}}\right), 4.31\left(\mathrm{q}, 2 \mathrm{H},{ }^{3} \mathrm{~J}_{\mathrm{Hb}-\mathrm{Hc}}=7.1 \mathrm{~Hz}, \mathrm{H}_{\mathrm{b}}\right), 3.96\left(\mathrm{~m}, 2 \mathrm{H}, \mathrm{H}_{\mathrm{h}}\right), 3.88\left(\mathrm{~s}, 3 \mathrm{H}, \mathrm{H}_{\mathrm{e}}\right), 2.95$ $\left(\mathrm{m}, 2 \mathrm{H}, \mathrm{H}_{\mathrm{f}}\right), 2.14\left(\mathrm{~m}, 2 \mathrm{H}, \mathrm{H}_{\mathrm{g}}\right), 1.35\left(\mathrm{t}, 3 \mathrm{H},{ }^{3} \mathrm{~J}_{\mathrm{Hc}-\mathrm{Hb}}=7.1 \mathrm{~Hz}, \mathrm{H}_{\mathrm{c}}\right) ;{ }^{13} \mathrm{C} \mathrm{NMR}\left(100 \mathrm{MHz}, \mathrm{CDCl}_{3}\right) \delta 166.3$ $\left(C_{a}\right), 153.6\left(C_{4^{\prime}}\right), 149.2\left(C_{4}\right), 145.4\left(C_{2}\right), 137.5\left(C_{8 a}\right), 135.7\left(C_{1^{\prime}}\right), 131.8\left(C_{7}\right), 129.7\left(C_{5}\right), 129.6\left(C_{2^{\prime}}\right)$, $129.1\left(C_{8}\right), 127.1\left(C_{6^{\prime}}\right), 126.4\left(C_{4 a}\right), 122.2\left(C_{3^{\prime}}\right), 119.0(C N), 122.1\left(C_{5^{\prime}}\right), 107.5\left(C_{6}\right), 106.0\left(C_{3}\right)$, $61.1\left(C_{b}\right), 56.4\left(C_{e}\right), 55.5\left(C_{d}\right), 51.7\left(C_{h}\right), 26.8\left(C_{f}\right), 20.9\left(C_{g}\right), 14.5\left(C_{c}\right)$; HRMS (ESI) m/z calculated for $\mathrm{C}_{24} \mathrm{H}_{23}{ }^{35} \mathrm{CIN}_{3} \mathrm{O}_{3}[\mathrm{M}+\mathrm{H}]^{+}:$: 436.1428, found $\mathrm{C}_{24} \mathrm{H}_{23}{ }^{35} \mathrm{CIN}_{3} \mathrm{O}_{3}[\mathrm{M}+\mathrm{H}]^{+}: 436.1423$ (100\%); $\mathrm{C}_{24} \mathrm{H}_{23}{ }^{37} \mathrm{ClN}_{3} \mathrm{O}_{3}[\mathrm{M}+\mathrm{H}]^{+}: 438.1433$ (33\%).

\subsubsection{Ethyl 8-bromo-4-[(tert-butoxycarbonyl)(3-chloro-4-methoxybenzyl)amino] -6- cyanoquinoline-3-carboxylate (18):}

Di-tert-butyl dicarbonate (0.92 g, $4.22 \mathrm{mmol}, 2.0$ eq.), triethylamine (59 $\mu \mathrm{L}, 4.22 \mathrm{mmol}, 2.0$ eq.) and $\mathrm{N}, \mathrm{N}$-4-dimethylaminopyridine $(51 \mathrm{mg}, 0.42 \mathrm{mmol}, 0.2 \mathrm{eq}$.) were added under argon to a solution of 14 [28] (1.0 g, $2.11 \mathrm{mmol}, 1.0$ eq.) in anhydrous tetrahydrofuran ( $40 \mathrm{~mL})$. The resulting mixture was stirred at $\mathrm{rt}$ for 2.5 hours before addition of water $(40 \mathrm{~mL})$. The aqueous layer was extracted with dichloromethane $(3 \times 50 \mathrm{~mL})$. The combined organic layers were washed with brine $(100 \mathrm{~mL})$, dried over magnesium sulfate, filtered and concentrated under reduced pressure. The crude residue was purified by column chromatography $\left(\mathrm{SiO}_{2}\right.$, ethyl acetate/cyclohexane, $3 / 7, \mathrm{v} / \mathrm{v})$ to give the desired product $(0.90 \mathrm{~g}, 1.57 \mathrm{mmol}, 74 \%)$ as a white solid. $\mathrm{R}_{\mathrm{f}}\left(\mathrm{SiO}_{2}\right.$, ethyl acetate/cyclohexane, 3/7, v/v): 0.48; $\mathrm{mp}: 139 \pm 1{ }^{\circ} \mathrm{C} ; \mathrm{IR}\left(\mathrm{cm}^{-1}\right) \vee 2234\left(\mathrm{v}_{\mathrm{C} \equiv \mathrm{N}}\right)$, $1708\left(v_{\mathrm{C}=0}\right), 1366\left(\delta_{\mathrm{CH} 3}\right), 1255\left(\mathrm{v}_{\mathrm{C}-\mathrm{O}}\right), 1151\left(\mathrm{v}_{\mathrm{C}-\mathrm{O}}\right)$; Analyses of NMR spectra revealed that this product was a mixture of two rotamers, named $A$ and $B$ with a ratio $A / B=1 / 0.3 ;{ }^{1} \mathrm{H}$ NMR (400 $\left.\mathrm{MHz}, \mathrm{CDCl}_{3}\right) \delta 9.41\left(\mathrm{~s}, 1 \mathrm{H}, \mathrm{H}_{2(\mathrm{~B})}\right), 9.40\left(\mathrm{~s}, 1 \mathrm{H}, \mathrm{H}_{2(\mathrm{~A})}\right), 8.03\left(\mathrm{~d}, 1 \mathrm{H},{ }^{4} J_{\mathrm{H} 5(\mathrm{~A})-\mathrm{H} 7(\mathrm{~A})}=1.7 \mathrm{~Hz}, \mathrm{H}_{5}(\mathrm{~A})\right), 8.02$ $\left(\mathrm{d}, 1 \mathrm{H},{ }^{4} J_{\mathrm{H} 5(\mathrm{~B})-\mathrm{H} 7(\mathrm{~B})}=1.6 \mathrm{~Hz}, \mathrm{H}_{5(\mathrm{~B})}\right), 7.77\left(\mathrm{~d}, 1 \mathrm{H},{ }^{4} J_{\mathrm{H} 7(\mathrm{~B})-\mathrm{H} 5(\mathrm{~B})}=1.6 \mathrm{~Hz}, \mathrm{H}_{7(\mathrm{~B})}\right), 7.75\left(\mathrm{~d}, 1 \mathrm{H},{ }^{4} \mathrm{~J}_{\mathrm{H} 7(\mathrm{~A})-\mathrm{H} 5(\mathrm{~A})}\right.$ 
$\left.=1.7 \mathrm{~Hz}, \mathrm{H}_{7(A)}\right), 6.98\left(\mathrm{~d}, 1 \mathrm{H},{ }^{4} \mathrm{~J}_{\mathrm{H} 2^{\prime}(B)-H 6^{\prime}(B)}=1.9 \mathrm{~Hz}, \mathrm{H}_{2^{\prime}(B)}\right), 6.91\left(\mathrm{~d}, 1 \mathrm{H},{ }^{4} \mathrm{~J}_{\mathrm{H} 2^{\prime}(\mathrm{A})-\mathrm{H}^{\prime}(\mathrm{A})}=2.0 \mathrm{~Hz}, \mathrm{H}_{2^{\prime}(\mathrm{A})}\right)$, $6.89\left(\mathrm{dd}, 1 \mathrm{H},{ }^{4} J_{\mathrm{H} 6^{\prime}(\mathrm{A})-\mathrm{H} 2^{\prime}(\mathrm{A})}=2.0 \mathrm{~Hz},{ }^{3} J_{\mathrm{H} 6^{\prime}(\mathrm{A})-\mathrm{H} 5^{\prime}(\mathrm{A})}=8.4 \mathrm{~Hz}, \mathrm{H}_{6^{\prime}(\mathrm{A})}\right), 6.81\left(\mathrm{dd}, 1 \mathrm{H},{ }^{4} J_{\mathrm{H} 6^{\prime}(\mathrm{B})-\mathrm{H} 2^{\prime}(\mathrm{B})}=1.9 \mathrm{~Hz}\right.$, $\left.{ }^{3} J_{H 6^{\prime}(B)-H 5^{\prime}(B)}=8.4 \mathrm{~Hz}, \mathrm{H}_{6^{\prime}(B)}\right), 6.67\left(\mathrm{~m}, 1 \mathrm{H}, \mathrm{H}_{5^{\prime}(\mathrm{A} / \mathrm{B})}\right), 5.00\left(\mathrm{~d}, 1 \mathrm{H},{ }^{2} J_{H d(A)-H d^{\prime}(\mathrm{A})}=14.6 \mathrm{~Hz}, \mathrm{H}_{\mathrm{d}(\mathrm{A})}\right), 4.89$ $\left(\mathrm{d}, 1 \mathrm{H},{ }^{2} J_{\mathrm{Hd}(\mathrm{B})-\mathrm{Hd}^{\prime}(B)}=14.9 \mathrm{~Hz}, \mathrm{H}_{\mathrm{d}(\mathrm{B})}\right), 4.25\left(\mathrm{~m}, 3 \mathrm{H}, \mathrm{H}_{\mathrm{b}(\mathrm{A} / \mathrm{B})}, \mathrm{H}_{\mathrm{d}^{\prime}(B)}\right), 4.14\left(\mathrm{~d}, 1 \mathrm{H},{ }^{2} \mathrm{~J}_{\mathrm{Hd}^{\prime}(\mathrm{A})-\mathrm{Hd}(\mathrm{A})}=14.6 \mathrm{~Hz}\right.$, $\left.H_{d^{\prime}(A)}\right), 3.71\left(s, 3 H, H_{h(B)}\right), 3.69\left(s, 3 H, H_{h(A)}\right), 1.50\left(s, 9 H, H_{g(B)}\right), 1.29\left(t, 3 H,{ }^{3}\right)_{H c(A / B)}-H b(A / B)=7.1 \mathrm{~Hz}$, $\left.\mathrm{H}_{\mathrm{c}(\mathrm{A} / \mathrm{B})}\right), 1.08\left(\mathrm{~s}, 9 \mathrm{H}, \mathrm{H}_{\mathrm{g}(\mathrm{A})}\right) ;{ }^{13} \mathrm{C} \mathrm{NMR}\left(100 \mathrm{MHz}, \mathrm{CDCl}_{3}\right) \delta 163.2\left(\mathrm{C}_{\mathrm{a}(\mathrm{A})}\right), 162.9\left(\mathrm{C}_{\mathrm{a}(\mathrm{B})}\right), 154.8\left(\mathrm{C}_{2(\mathrm{~A} / \mathrm{B})}\right)$, $154.6\left(\mathrm{C}_{4^{\prime}(\mathrm{A} / \mathrm{B})}\right), 153.5\left(\mathrm{C}_{4(\mathrm{~B})}\right), 153.0\left(\mathrm{C}_{4(\mathrm{~A})}\right), 149.4\left(\mathrm{C}_{\mathrm{e}(\mathrm{B})}\right), 148.8\left(\mathrm{C}_{\mathrm{e}(\mathrm{A})}\right), 148.5\left(\mathrm{C}_{8 \mathrm{a}(\mathrm{B})}\right), 148.4\left(\mathrm{C}_{8 \mathrm{a}(\mathrm{A})}\right)$, $134.6\left(C_{5(\mathrm{~A} / \mathrm{B})}\right), 131.1\left(\mathrm{C}_{2^{\prime}(\mathrm{A} / \mathrm{B})}\right), 130.8\left(\mathrm{C}_{7(\mathrm{~B})}\right), 130.7\left(\mathrm{C}_{7(\mathrm{~A})}\right), 129.0\left(\mathrm{C}_{6^{\prime}(\mathrm{A} / \mathrm{B})}\right), 128.9\left(\mathrm{C}_{1^{\prime}(\mathrm{A} / \mathrm{B})}\right), 127.0$ $\left(C_{4 a(B)}\right), 126.9\left(C_{4 a(A)}\right), 126.2\left(C_{8(B)}\right), 126.1\left(C_{8(A)}\right), 123.4\left(C_{3(B)}\right), 122.8\left(C_{3(A)}\right), 121.9\left(C_{3^{\prime}(A / B)}\right), 116.4$ $\left(\mathrm{CN}_{(\mathrm{B})}\right), 116.3\left(\mathrm{CN}_{(\mathrm{A})}\right), 111.8\left(\mathrm{C}_{5^{\prime}(\mathrm{A})}\right), 111.7\left(\mathrm{C}_{5^{\prime}(\mathrm{B})}\right), 111.4\left(\mathrm{C}_{6(\mathrm{~B})}\right), 111.3\left(\mathrm{C}_{6(\mathrm{~A})}\right), 82.5\left(\mathrm{C}_{\mathrm{f}(\mathrm{B})}\right), 81.6$ $\left(C_{f(A)}\right), 62.2\left(C_{b(A)}\right), 62.0\left(C_{b(B)}\right), 55.8\left(C_{h(A / B)}\right), 54.3\left(C_{d(B)}\right), 53.2\left(C_{d(A)}\right), 28.1\left(3 C, C_{g(B)}\right), 27.7(3 C$, $\left.\mathrm{C}_{g(\mathrm{~A})}\right), 13.8\left(\mathrm{C}_{\mathrm{c}(\mathrm{A} / \mathrm{B})}\right)$; HRMS (ESI) $\mathrm{m} / z$ calculated for $\mathrm{C}_{26} \mathrm{H}_{26}{ }^{35} \mathrm{Cl}^{79} \mathrm{BrN}_{3} \mathrm{O}_{5}[\mathrm{M}+\mathrm{H}]^{+}: 574.0744$, found $\mathrm{C}_{26} \mathrm{H}_{26}{ }^{35} \mathrm{Cl}^{79} \mathrm{BrN}_{3} \mathrm{O}_{5}[\mathrm{M}+\mathrm{H}]^{+}: 574.0799$ (77\%); $\mathrm{C}_{26} \mathrm{H}_{26}{ }^{37} \mathrm{Cl}^{79} \mathrm{BrN}_{3} \mathrm{O}_{5}$ or $\mathrm{C}_{26} \mathrm{H}_{26}{ }^{35} \mathrm{Cl}^{81} \mathrm{BrN}_{3} \mathrm{O}_{5}[\mathrm{M}+\mathrm{H}]^{+}$: 576.0721 (100\%); $\mathrm{C}_{26} \mathrm{H}_{26}{ }^{37} \mathrm{Cl}^{81} \mathrm{BrN}_{3} \mathrm{O}_{5}[\mathrm{M}+\mathrm{H}]^{+}: 578.0730$ (24\%).

\subsubsection{Ethyl 4-[(tert-butoxycarbonyl)-(3-chloro-4-methoxybenzyl)amino]-6-cyano-8-(3- fluoropropyl)quinoline-3-carboxylate (19):}

Tetra- $n$-butylammonium fluoride (TBAF) solution (1.0 M in THF, $10 \mathrm{~mL}$ ) was placed into a threenecked flask under argon atmosphere. The solvent was removed under reduced pressure in order to obtain anhydrous and pure TBAF. 3-Bromopropene (1.39 mL, $16.1 \mathrm{mmol}, 1.0$ eq.) was added and the resulting mixture was stirred at room temperature for 30 minutes. The product was then collected in a weighed gas trap with an anhydrous argon stream flow as carrier for three hours to produce allyl fluoride $\left(310 \mathrm{mg}, 5.16 \mathrm{mmol}, 32 \%\right.$ ) as a colorless liquid at $-60{ }^{\circ} \mathrm{C}$ (see Figure $\mathrm{S} 1$ in supporting information).

Under stirring an $0.5 \mathrm{M}$ solution of 9-BBN in tetrahydrofuran (2.1 mL, $1.05 \mathrm{mmol}, 2.0$ eq.) was added at $-10^{\circ} \mathrm{C}$ to the allyl fluoride solution (10.0 eq.) in anhydrous and previously degassed tetrahydrofuran $(3 \mathrm{~mL})$. The resulting mixture was stirred at room temperature for 7 hours. This solution was added to a mixture of 18 (300 mg, $0.54 \mathrm{mmol}, 1.0$ eq.), tetrakis(triphenylphosphine)palladium(0) (60 mg, $51.9 \mu \mathrm{mol}, 0.1$ eq.), potassium carbonate (288 mg, $2.08 \mathrm{mmol}, 4.0$ eq.) and water $(0.3 \mathrm{~mL})$ in $\mathrm{N}, \mathrm{N}$-dimethylformamide $(20 \mathrm{~mL})$. The resulting mixture was stirred at $80^{\circ} \mathrm{C}$ for 16 hours. After cooling to room temperature, water $(30 \mathrm{~mL})$ was added and the aqueous layer was extracted with ethyl acetate $(3 \times 30 \mathrm{~mL})$. The combined organic layers were washed with brine $(100 \mathrm{~mL})$, dried over anhydrous magnesium sulfate, filtered and concentrated under reduced pressure. The crude residue was purified by column chromatography $\left(\mathrm{SiO}_{2}\right.$, ethyl acetate/cyclohexane, 1/9, v/v) to give a mixture of 18 and 19 which was used in the next step without further purification $(200 \mathrm{mg})$.

\subsubsection{Tert-butyl 3-chloro-4-methoxybenzyl[6-cyano-8-(3-fluoropropyl)-3- (hydroxymethyl)quinolin-4-yl]carbamate (20):}


A 1.1 M solution of lithium tri-tert-butoxyaluminum hydride in tetrahydrofuran $(0.8 \mathrm{~mL}, 0.88$ $\mathrm{mmol}$ ) was added to a mixture of crude $19(100 \mathrm{mg})$ in anhydrous tetrahydrofuran $(5 \mathrm{~mL})$ under stirring and argon atmosphere. The resulting mixture was stirred at $60^{\circ} \mathrm{C}$ for 20 hours. After cooling to room temperature, water $(20 \mathrm{~mL})$ was added under stirring. The solution was extracted with ethyl acetate $(3 \times 20 \mathrm{~mL})$ and then with dichloromethane $(2 \times 20 \mathrm{~mL})$. These two organic layers were separately washed with brine $(100 \mathrm{~mL})$. The combined organic layers were dried over anhydrous magnesium sulfate, filtered and concentrated under reduced pressure. The crude residue was purified by column chromatography $\left(\mathrm{SiO}_{2}\right.$, ethanol/dichloromethane, 5/95, v/v) to give the desired product ( $20 \mathrm{mg}, 38.9 \mu \mathrm{mol}$ ) as a white solid. Yield: 7\%; $\mathrm{R}_{\mathrm{f}}\left(\mathrm{SiO}_{2}\right.$, ethanol/dichloromethane, 5/95, v/v): 0.43; Analyses of NMR spectra revealed that this product was a mixture of two rotamers, named $A$ and $B$ with a ratio $A / B=$ 1/0.7; ${ }^{1} \mathrm{H}$ NMR (400 MHz, $\left.\mathrm{CDCl}_{3}\right) \delta 9.18\left(\mathrm{~s}, 1 \mathrm{H}, \mathrm{H}_{2(\mathrm{~B})}\right), 9.15\left(\mathrm{~s}, 1 \mathrm{H}, \mathrm{H}_{2(\mathrm{~A})}\right), 8.08\left(\mathrm{~m}, 1 \mathrm{H}, \mathrm{H}_{5(\mathrm{~A})}\right), 7.85$ $\left(m, 1 \mathrm{H}, \mathrm{H}_{5(B)}\right), 7.75\left(m, 1 \mathrm{H}, \mathrm{H}_{7(A)}\right), 7.72\left(m, 1 \mathrm{H}, \mathrm{H}_{7(B)}\right), 7.13\left(\mathrm{~m}, 1 \mathrm{H}, \mathrm{H}_{2^{\prime}(A)}\right), 7.07\left(m, 1 \mathrm{H}, \mathrm{H}_{2^{\prime}(B)}\right)$, $7.04\left(\mathrm{~m}, 1 \mathrm{H}, \mathrm{H}_{6^{\prime}(B)}\right), 6.82\left(\mathrm{~m}, 3 \mathrm{H}, \mathrm{H}_{6^{\prime}(\mathrm{A})}, \mathrm{H}_{5^{\prime}(B)}, \mathrm{H}_{5^{\prime}(\mathrm{A})}\right), 5.17\left(\mathrm{~d}, 1 \mathrm{H},{ }^{2} \mathrm{~J}_{\mathrm{He}(\mathrm{A})-\mathrm{He}^{\prime}(\mathrm{A})}=14.7 \mathrm{~Hz}, \mathrm{H}_{\mathrm{e}(\mathrm{A})}\right), 5.08$ $\left(\mathrm{d}, 1 \mathrm{H},{ }^{2} J_{\mathrm{He}(\mathrm{B})-\mathrm{He}^{\prime}(\mathrm{B})}=14.8 \mathrm{~Hz}, \mathrm{H}_{\mathrm{e}(\mathrm{B})}\right), 4.55\left(\mathrm{dt}, 2 \mathrm{H},{ }^{3} J_{\mathrm{Hi}(\mathrm{A})-\mathrm{Hh}(\mathrm{A})}=5.6 \mathrm{~Hz},{ }^{2} J_{\mathrm{Hi}(\mathrm{A})-\mathrm{F}}=47.3 \mathrm{~Hz}, \mathrm{H}_{\mathrm{i}(\mathrm{A})}\right), 4.36-$ $4.29\left(\mathrm{~m}, 3 \mathrm{H}, \mathrm{H}_{\mathrm{i}(\mathrm{B})}, \mathrm{H}_{\mathrm{e}(\mathrm{A})}, \mathrm{H}_{\mathrm{e}(\mathrm{B})}\right), 4.17\left(\mathrm{~m}, 4 \mathrm{H}, \mathrm{H}_{\mathrm{a}(\mathrm{A})}, \mathrm{H}_{\mathrm{a}(\mathrm{B})}\right), 3.90\left(\mathrm{~s}, 3 \mathrm{H}, \mathrm{H}_{\mathrm{f}(\mathrm{B})}\right), 3.89\left(\mathrm{~s}, 3 \mathrm{H}, \mathrm{H}_{\mathrm{f}(\mathrm{A})}\right), 3.44$ $\left(\mathrm{m}, 4 \mathrm{H}, \mathrm{H}_{\mathrm{g}(\mathrm{A})}, \mathrm{H}_{\mathrm{g}(\mathrm{B})}\right), 2.22\left(\mathrm{~m}, 4 \mathrm{H}, \mathrm{H}_{\mathrm{h}(\mathrm{A})}, \mathrm{H}_{\mathrm{h}(\mathrm{B})}\right), 1.65\left(\mathrm{~s}, 9 \mathrm{H}, \mathrm{H}_{\mathrm{d}(\mathrm{A})}\right), 1.26\left(\mathrm{~s}, 9 \mathrm{H}, \mathrm{H}_{\mathrm{d}(\mathrm{B})}\right) ; \mathrm{HRMS}(\mathrm{ESI})$ $\mathrm{m} / \mathrm{z}$ calculated for $\mathrm{C}_{27} \mathrm{H}_{30}{ }^{35} \mathrm{ClFN}_{3} \mathrm{O}_{4}[\mathrm{M}+\mathrm{H}]^{+}: 514.1909$, found $\mathrm{C}_{27} \mathrm{H}_{30}{ }^{35} \mathrm{ClFN}_{3} \mathrm{O}_{4}[\mathrm{M}+\mathrm{H}]^{+}: 514.1953$ (100\%); $\mathrm{C}_{27} \mathrm{H}_{30}{ }^{37} \mathrm{ClFN}_{3} \mathrm{O}_{4}[\mathrm{M}+\mathrm{H}]^{+}: 516.1985$ (33\%).

\subsubsection{4-[(3-Chloro-4-methoxybenzyl)amino]-8-(3-fluoropropyl)-3- (hydroxymethyl)quinoline-6-carbonitrile (5):}

Trifluoroacetic acid $(1.0 \mathrm{~mL}$ ) was added to a solution of 20 (20 mg, $38.9 \mu \mathrm{mol}, 1.0$ eq.) in anhydrous dichloromethane $(2 \mathrm{~mL})$ under argon atmosphere. The resulting mixture was stirred at $r$ for 3 hours and the solvent was evaporated under reduced pressure. The crude residue was purified by column chromatography $\left(\mathrm{SiO}_{2}\right.$, ethanol/dichloromethane, 5/95, v/v) to give the desired product $(5.0 \mathrm{mg}, 12.1 \mu \mathrm{mol}, 31 \%)$ as a white solid; $\mathrm{R}_{\mathrm{f}}\left(\mathrm{SiO}_{2}\right.$, ethanol/dichloromethane, 5/95, v/v): 0.19; IR $\left(\mathrm{cm}^{-1}\right) \vee 3412\left(\mathrm{v}_{\mathrm{N}-\mathrm{H}}\right), 2229\left(\mathrm{v}_{\mathrm{C} \equiv \mathrm{N}}\right), 1503\left(\delta_{\mathrm{N}-\mathrm{H}}\right)$, $1259\left(\mathrm{v}_{\mathrm{c}-\mathrm{o}}\right), 1064\left(\mathrm{v}_{\mathrm{c}-\mathrm{O}}\right), 1020\left(\mathrm{v}_{\mathrm{C}-\mathrm{F}}\right) ;{ }^{1} \mathrm{H}$ NMR $\left(400 \mathrm{MHz}, \mathrm{CDCl}_{3}\right) \delta 8.53\left(\mathrm{~s}, 1 \mathrm{H}, \mathrm{H}_{2}\right), 8.35(\mathrm{~d}, 1 \mathrm{H}$, $\left.{ }^{4} J_{\mathrm{H} 5-\mathrm{H} 7}=1.7 \mathrm{~Hz}, \mathrm{H}_{5}\right), 7.64\left(\mathrm{~d}, 1 \mathrm{H},{ }^{4} J_{\mathrm{H} 7-\mathrm{H} 5}=1.7 \mathrm{~Hz}, \mathrm{H}_{7}\right), 7.37\left(\mathrm{~d}, 1 \mathrm{H},{ }^{4} J_{\mathrm{Hz}^{\prime}-\mathrm{H}^{\prime}}=2.2 \mathrm{~Hz}, \mathrm{H}_{2^{\prime}}\right), 7.21$ (dd, $\left.1 \mathrm{H},{ }^{4} J_{\mathrm{H} 6^{\prime}-\mathrm{H} 2^{\prime}}=2.2 \mathrm{~Hz},{ }^{3} \mathrm{JH}^{\prime}-\mathrm{H}^{\prime}=8.4 \mathrm{~Hz}, \mathrm{H}_{6^{\prime}}\right), 6.94\left(\mathrm{~d}, 1 \mathrm{H},{ }^{3} \mathrm{~J}_{\mathrm{H} 5^{\prime}-\mathrm{H} 6^{\prime}}=8.4 \mathrm{~Hz}, \mathrm{H}_{5^{\prime}}\right), 4.77\left(\mathrm{~m}, 4 \mathrm{H}, \mathrm{H}_{\mathrm{a}}, \mathrm{H}_{\mathrm{b}}\right)$, $4.53\left(\mathrm{td}, 2 \mathrm{H},{ }^{3} \mathrm{Hff}_{\mathrm{He}}=5.9 \mathrm{~Hz},{ }^{2} \mathrm{JHf}_{\mathrm{H}}=47.2 \mathrm{~Hz}, \mathrm{H}_{\mathrm{f}}\right), 3.92\left(\mathrm{~s}, 3 \mathrm{H}, \mathrm{H}_{\mathrm{c}}\right), 3.32\left(\mathrm{~m}, 2 \mathrm{H}, \mathrm{H}_{\mathrm{d}}\right), 2.16(\mathrm{~m}, 2 \mathrm{H}$, $\mathrm{He}_{\mathrm{e}}$; HRMS (ESI) $\mathrm{m} / z$ calculated for $\mathrm{C}_{22} \mathrm{H}_{22}{ }^{35} \mathrm{ClFN}_{3} \mathrm{O}_{2}[\mathrm{M}+\mathrm{H}]^{+}: 414.1385$, found $\mathrm{C}_{22} \mathrm{H}_{22}{ }^{35} \mathrm{ClFN}_{3} \mathrm{O}_{2}$ $[\mathrm{M}+\mathrm{H}]^{+}: 414.1357$ (100\%); $\mathrm{C}_{22} \mathrm{H}_{22}{ }^{37} \mathrm{ClFN}_{3} \mathrm{O}_{2}[\mathrm{M}+\mathrm{H}]^{+}: 416.1244$ (33\%).

\subsubsection{Ethyl 4-[(3-chloro-4-methoxybenzyl)amino]-6-cyano-8-(4-fluoropiperidin-1-yl) quinoline-3-carboxylate (21a):}

4-Fluoropiperidine hydrochloride (133 mg, $0.95 \mathrm{mmol}, 3.0$ eq.), palladium(II) acetate $(7.1 \mathrm{mg}$, $31.6 \mu \mathrm{mol}, 0.1$ eq.), caesium carbonate ( $618 \mathrm{mg}, 1.90 \mathrm{mmol}, 6.0$ eq.) and BINAP (39 mg, 62.6 $\mu \mathrm{mol}, 0.2$ eq.) were successively added to a solution of 14 [28] (150 mg, $0.32 \mathrm{mmol}, 1$ eq.) in anhydrous toluene $(10 \mathrm{~mL})$ in a sealed flask under stirring and argon atmosphere. The resulting 
mixture was refluxed for 3 hours. After cooling to room temperature, the mixture was filtered thought Celite 545 and washed with dichloromethane $(60 \mathrm{~mL})$. The filtrate was concentrated under reduced pressure and the crude product was purified by column chromatography $\left(\mathrm{SiO}_{2}\right.$, ethyl acetate/cyclohexane, 4/6, v/v) to give the desired product ( $90 \mathrm{mg}, 0.18 \mathrm{mmol}, 56 \%$ ) as a light yellow solid. $\mathrm{R}_{\mathrm{f}}\left(\mathrm{SiO}_{2}\right.$, ethyl acetate/cyclohexane, 4/6, v/v): 0.24; $\mathrm{Mp}: 110 \pm 1{ }^{\circ} \mathrm{C}$; IR (cm ${ }^{-}$ $\left.{ }^{1}\right)$ v $2226\left(\mathrm{v}_{\mathrm{C} \equiv \mathrm{N}}\right), 1669\left(\mathrm{v}_{\mathrm{C}=\mathrm{O}}\right), 1502\left(\delta_{\mathrm{N}-\mathrm{H}}\right), 1261\left(\mathrm{v}_{\mathrm{C}-\mathrm{O}}\right), 1183\left(\mathrm{v}_{\mathrm{C}-\mathrm{O}}\right), 1027\left(\mathrm{v}_{\mathrm{C}-\mathrm{F}}\right) ;{ }^{1} \mathrm{H}$ NMR $(400 \mathrm{MHz}$, $\left.\mathrm{CDCl}_{3}\right) \delta 9.47$ (brs, $\left.1 \mathrm{H}, \mathrm{NH}\right), 9.19\left(\mathrm{~s}, 1 \mathrm{H}, \mathrm{H}_{2}\right), 8.07\left(\mathrm{~d}, 1 \mathrm{H},{ }^{4} J_{\mathrm{H} 5-\mathrm{H} 7}=1.2 \mathrm{~Hz}, \mathrm{H}_{5}\right), 7.38\left(\mathrm{~d}, 1 \mathrm{H},{ }^{4} \mathrm{H}_{\mathrm{H}^{\prime}-}\right.$ $\left.\mathrm{H}^{\prime}=2.2 \mathrm{~Hz}, \mathrm{H}_{2^{\prime}}\right), 7.24\left(\mathrm{dd}, 1 \mathrm{H},{ }^{3} \mathrm{JH}_{\mathrm{H}^{\prime}-\mathrm{H} 5^{\prime}}=8.4 \mathrm{~Hz},{ }^{4} \mathrm{JH}^{\prime}-\mathrm{H}^{\prime}=2.2 \mathrm{~Hz}, \mathrm{H}_{6^{\prime}}\right), 7.21\left(\mathrm{~m}, 1 \mathrm{H}, \mathrm{H}_{7}\right), 6.96(\mathrm{~d}$, $\left.1 \mathrm{H},{ }^{3} J_{\mathrm{H}^{\prime}-\mathrm{H} 6^{\prime}}=8.4 \mathrm{~Hz}, \mathrm{H}_{5^{\prime}}\right), 4.93\left(\mathrm{~m}, 1 \mathrm{H}, \mathrm{H}_{\mathrm{h}}\right), 4.82\left(\mathrm{~d}, 2 \mathrm{H},{ }^{3} \mathrm{~J}_{\mathrm{Hd}-\mathrm{NH}}=5.6 \mathrm{~Hz}, \mathrm{H}_{\mathrm{d}}\right), 4.37\left(\mathrm{q}, 2 \mathrm{H},{ }^{3} \mathrm{~J}_{\mathrm{Hb}-\mathrm{Hc}}\right.$ $\left.=7.1 \mathrm{~Hz}, \mathrm{H}_{\mathrm{b}}\right), 3.91\left(\mathrm{~s}, 3 \mathrm{H}, \mathrm{H}_{\mathrm{e}}\right), 3.40\left(\mathrm{~m}, 4 \mathrm{H}, \mathrm{H}_{\mathrm{f}}, \mathrm{H}_{\mathrm{f}^{\prime}}\right), 2.15\left(\mathrm{~m}, 4 \mathrm{H}, \mathrm{H}_{\mathrm{g}}, \mathrm{H}_{\mathrm{g}^{\prime}}\right), 1.40\left(\mathrm{t}, 3 \mathrm{H},{ }^{3} \mathrm{~J}_{\mathrm{Hc}-\mathrm{Hb}}=7.1\right.$ $\left.\mathrm{Hz}, \mathrm{H}_{\mathrm{c}}\right) ;{ }^{13} \mathrm{C} \operatorname{NMR}\left(100 \mathrm{MHz}, \mathrm{CDCl}_{3}\right) \delta 168.2\left(\mathrm{C}_{\mathrm{a}}\right), 157.0\left(\mathrm{C}_{4}\right), 154.9\left(\mathrm{C}_{4^{\prime}}\right), 151.7\left(\mathrm{C}_{2}\right), 150.8\left(\mathrm{C}_{8}\right)$, $147.5\left(C_{8 \mathrm{a}}\right), 130.7\left(\mathrm{C}_{1^{\prime}}\right), 129.3\left(\mathrm{C}_{2^{\prime}}\right), 126.8\left(\mathrm{C}_{6^{\prime}}\right), 125.0\left(\mathrm{C}_{5}\right), 123.1\left(\mathrm{C}_{3^{\prime}}\right), 120.0\left(\mathrm{C}_{4 \mathrm{a}}\right), 119.3(\mathrm{CN})$, $118.1\left(C_{7}\right), 112.5\left(C_{5^{\prime}}\right), 107.4\left(C_{6}\right), 104.8\left(C_{3}\right), 88.1\left(d,{ }^{1} J_{C h-F}=170 \mathrm{~Hz}, C_{h}\right), 61.2\left(C_{b}\right), 56.2\left(C_{e}\right)$, $52.1\left(C_{d}\right), 47.9\left(d, 2 C,{ }^{3} J_{C f / f^{\prime}-F}=5 ~ H z, C_{f}, C_{f^{\prime}}\right), 31.5\left(d, 2 C,{ }^{2} J_{C g} / g^{\prime}-F=20 ~ H z, C_{g}, C_{g^{\prime}}\right), 14.3\left(C_{c}\right) ; H R M S$ (ESI) $\mathrm{m} / z$ calculated for $\mathrm{C}_{26} \mathrm{H}_{27}{ }^{35} \mathrm{ClFN}_{4} \mathrm{O}_{3}[\mathrm{M}+\mathrm{H}]^{+}:$: 497.1756, found $\mathrm{C}_{26} \mathrm{H}_{27}{ }^{35} \mathrm{CIFN}_{4} \mathrm{O}_{3}[\mathrm{M}+\mathrm{H}]^{+}$: 497.1751 (100\%); $\mathrm{C}_{26} \mathrm{H}_{27}{ }^{37} \mathrm{CIFN}_{4} \mathrm{O}_{3}[\mathrm{M}+\mathrm{H}]^{+}: 499.1850$ (33\%).

\subsubsection{Ethyl 4-[(3-chloro-4-methoxybenzyl)amino]-6-cyano-8-(3-fluoroazetidin-1- yl)quinoline-3-carboxylate (21b):}

In a sealed flask compound 14 [28] $(0.26 \mathrm{~g}, 0.55 \mathrm{mmol}, 1.0$ eq.) was diluted in anhydrous toluene $(20 \mathrm{~mL}$ ) and 3-fluoroazetidine hydrochloride ( $91 \mathrm{mg}, 0.82 \mathrm{mmol}, 1.5$ eq.), palladium(II) acetate (12 mg, $53.4 \mu \mathrm{mol}, 0.1$ eq.), caesium carbonate $(0.71 \mathrm{~g}, 2.18 \mathrm{mmol}, 4.0$ eq.), and BINAP ( $68 \mathrm{mg}, 0.11 \mathrm{mmol}, 0.2$ eq.) were successively added under argon atmosphere. The resulting mixture was stirred at $120^{\circ} \mathrm{C}$ for 3 hours. After cooling to room temperature, the mixture was filtered on Celite 545 and washed with ethyl acetate $(40 \mathrm{~mL})$. The filtrate was concentrated under reduced pressure and the crude residue was purified by column chromatography $\left(\mathrm{SiO}_{2}\right.$, ethyl acetate/cyclohexane, $2 / 8, \mathrm{v} / \mathrm{v})$ to give the desired product $(0.20 \mathrm{~g}, 0.43 \mathrm{mmol}, 78 \%)$ as a yellow solid. $\mathrm{R}_{\mathrm{f}}\left(\mathrm{SiO}_{2}\right.$, cyclohexane/ethyl acetate, 7/3, v/v): 0.41; $\mathrm{Mp}: 155 \pm 1{ }^{\circ} \mathrm{C} ; \mathrm{IR}\left(\mathrm{cm}^{-1}\right) \mathrm{v}$ $2227\left(v_{\mathrm{C} \equiv N}\right), 1670\left(v_{\mathrm{C}=\mathrm{O}}\right), 1590\left(\delta_{\mathrm{N}-\mathrm{H}}\right), 1504\left(\mathrm{v}_{\mathrm{C}=\mathrm{C}}\right), 1437\left(\delta_{\mathrm{CH} 2}\right), 1067\left(\mathrm{v}_{\mathrm{C}-\mathrm{O}}\right), 1180\left(\mathrm{v}_{\mathrm{C}-\mathrm{O}}\right), 1066\left(\mathrm{v}_{\mathrm{C}-}\right.$ o), $1024\left(\mathrm{v}_{\mathrm{C}-\mathrm{F}}\right) ;{ }^{1} \mathrm{H}$ NMR $\left(400 \mathrm{MHz}, \mathrm{CDCl}_{3}\right) \delta 9.40$ (brs, $\left.1 \mathrm{H}, \mathrm{NH}\right), 9.02\left(\mathrm{~s}, 1 \mathrm{H}, \mathrm{H}_{2}\right), 7.79\left(\mathrm{~d}, 1 \mathrm{H},{ }^{4} \mathrm{~J}_{\mathrm{H} 5-}\right.$ $\left.\mathrm{H}^{2}=1.4 \mathrm{~Hz}, \mathrm{H}_{5}\right), 7.36\left(\mathrm{~d}, 1 \mathrm{H},{ }^{4} J_{\mathrm{H} 2^{\prime}-\mathrm{H} 6^{\prime}}=2.2 \mathrm{~Hz}, \mathrm{H}_{2^{\prime}}\right), 7.23\left(\mathrm{dd}, 1 \mathrm{H},{ }^{3} \mathrm{JH}_{\mathrm{H}^{\prime}-\mathrm{H} 5^{\prime}}=8.4 \mathrm{~Hz},{ }^{4} J_{\mathrm{H} 6^{\prime}-\mathrm{H} 2^{\prime}}=2.2\right.$ $\left.\mathrm{Hz}, \mathrm{H}_{6^{\prime}}\right), 6.94\left(\mathrm{~d}, 1 \mathrm{H},{ }^{4} J_{\mathrm{H} 5^{\prime}-\mathrm{H} 6^{\prime}}=8.4 \mathrm{~Hz}, \mathrm{H}_{5^{\prime}}\right), 6.57\left(\mathrm{~d}, 1 \mathrm{H},{ }^{4} J_{\mathrm{H} 7-\mathrm{H} 5}=1.4 \mathrm{~Hz}, \mathrm{H}_{7}\right), 5.44\left(\mathrm{~m}, 1 \mathrm{H}, \mathrm{H}_{\mathrm{g}}\right), 4.79$ $\left(\mathrm{d}, 2 \mathrm{H},{ }^{3} \mathrm{~J}_{\mathrm{Hd}-\mathrm{NH}}=5.5 \mathrm{~Hz}, \mathrm{H}_{\mathrm{d}}\right), 4.61\left(\mathrm{~m}, 2 \mathrm{H}, \mathrm{H}_{\mathrm{f}}\right.$ or $\left.\mathrm{H}^{\prime}\right), 4.35\left(\mathrm{q}, 2 \mathrm{H},{ }^{3} \mathrm{~J}_{\mathrm{Hb}-\mathrm{Hc}}=7.1 \mathrm{~Hz}, \mathrm{H}_{\mathrm{b}}\right), 4.27(\mathrm{~m}, 2 \mathrm{H}$, $\mathrm{H}_{\mathrm{f}}$ or $\left.\mathrm{H}_{\mathrm{f}^{\prime}}\right), 3.90\left(\mathrm{~s}, 3 \mathrm{H}, \mathrm{H}_{\mathrm{e}}\right), 1.39\left(\mathrm{t}, 3 \mathrm{H},{ }^{3} \mathrm{~J}_{\mathrm{Hc}-\mathrm{Hb}}=7.1 \mathrm{~Hz}, \mathrm{H}_{\mathrm{c}}\right) ;{ }^{13} \mathrm{C} \mathrm{NMR}\left(100 \mathrm{MHz}, \mathrm{CDCl}_{3}\right) \delta 168.4$ $\left(C_{a}\right), 156.3\left(C_{4}\right), 154.9\left(C_{4^{\prime}}\right), 150.6\left(C_{2}\right), 148.2\left(C_{8}\right), 144.5\left(C_{8 a}\right), 130.9\left(C_{1^{\prime}}\right), 129.4\left(C_{2^{\prime}}\right), 126.9\left(C_{6^{\prime}}\right)$, $123.1\left(C_{3^{\prime}}\right), 120.7\left(C_{5}\right), 119.5\left(2 \mathrm{C}, \mathrm{CN}, \mathrm{C}_{4 \mathrm{a}}\right), 112.5\left(\mathrm{C}_{5^{\prime}}\right), 110.9\left(\mathrm{C}_{7}\right), 107.7\left(\mathrm{C}_{6}\right), 104.9\left(\mathrm{C}_{3}\right), 83.4(\mathrm{~d}$, $\left.{ }^{1} J_{C g-F}=201 \mathrm{~Hz}, C_{g}\right), 62.1\left(C_{f}\right.$ or $\left.C_{f^{\prime}}\right), 61.9\left(C_{f}\right.$ or $\left.C_{f^{\prime}}\right), 61.2\left(C_{b}\right), 56.3\left(C_{e}\right), 51.9\left(C_{d}\right), 14.3\left(C_{c}\right)$; HRMS (ESI) $\mathrm{m} / z$ calculated for $\mathrm{C}_{24} \mathrm{H}_{23}{ }^{35} \mathrm{CIFN}_{4} \mathrm{O}_{3}[\mathrm{M}+\mathrm{H}]^{+}: 469.1443$, found $\mathrm{C}_{24} \mathrm{H}_{23}{ }^{35} \mathrm{CIFN}_{4} \mathrm{O}_{3}[\mathrm{M}+\mathrm{H}]^{+}$: 469.1453 (100\%); $\mathrm{C}_{24} \mathrm{H}_{23}{ }^{37} \mathrm{CIFN}_{4} \mathrm{O}_{3}[\mathrm{M}+\mathrm{H}]^{+}: 471.1489$ (33\%). 


\subsubsection{Ethyl 4-[(3-chloro-4-methoxybenzyl)amino]-6-cyano-8-[((6-fluoro-5- methoxypyridin-2-yl)methyl)amino]quinoline-3-carboxylate (21c):}

The crude product $10 \mathrm{a}$ ( $2.0 \mathrm{~g}$, synthesis in supporting information) was dissolved in water (20 $\mathrm{mL}$ ) before addition of a saturated aqueous sodium bicarbonate solution ( $20 \mathrm{~mL}, \mathrm{pH} 7 / 8)$. This aqueous mixture was extracted with dichloromethane $(5 \times 30 \mathrm{~mL})$. The combined organic layers were washed with brine $(100 \mathrm{~mL})$, dried over magnesium sulfate, filtered and concentrated under reduced pressure. The obtained residue $(350 \mathrm{mg})$ was dissolved in anhydrous toluene $(3 \mathrm{~mL})$ under argon atmosphere. The resulting solution was added to a mixture of 14 [28] ( $0.30 \mathrm{~g}, 0.63 \mathrm{mmol}, 1.0 \mathrm{eq}$.), palladium(II) acetate (14 mg, $62.4 \mu \mathrm{mol}, 0.1$ eq.), caesium carbonate (1.03 g, $3.16 \mathrm{mmol}, 5.0$ eq.) and BINAP (79 mg, $0.13 \mathrm{mmol}, 0.2 \mathrm{eq}$.) in anhydrous toluene $(10 \mathrm{~mL})$ under argon atmosphere. The resulting mixture was refluxed for 6 hours. After cooling to room temperature, the mixture was filtered thought a Celite 545 pad and washed with dichloromethane $(100 \mathrm{~mL})$. The filtrate was concentrated under reduced pressure and the crude product was purified by column chromatography $\left(\mathrm{SiO}_{2}\right.$, ethyl acetate/cyclohexane, 3/7, v/v) to give the desired product (200 $\mathrm{mg}, 0.36 \mathrm{mmol}, 58 \%$ ) as a yellow solid. $\mathrm{R}_{\mathrm{f}}\left(\mathrm{SiO}_{2}\right.$, ethyl acetate/cyclohexane, 3/7, v/v): 0.19; $\mathrm{Mp}: 175 \pm 1^{\circ} \mathrm{C} ; \mathrm{IR}\left(\mathrm{cm}^{-1}\right) \mathrm{v}$ $2231\left(v_{\mathrm{C} \equiv N}\right), 1670\left(v_{\mathrm{C}=\mathrm{O}}\right), 1505\left(\delta_{\mathrm{N}-\mathrm{H}}\right), 1265\left(\mathrm{v}_{\mathrm{C}-\mathrm{O}}\right), 1181\left(\mathrm{v}_{\mathrm{C}-\mathrm{O}}\right), 1065\left(\mathrm{v}_{\mathrm{C}-\mathrm{O}}\right), 1022\left(\mathrm{v}_{\mathrm{C}-\mathrm{F}}\right) ;{ }^{1} \mathrm{H}$ NMR $\left(400 \mathrm{MHz}, \mathrm{CDCl}_{3}\right) \delta 9.55\left(\mathrm{t}, 1 \mathrm{H},{ }^{3} \mathrm{~J}_{\mathrm{Hd}-\mathrm{He}}=5.2 \mathrm{~Hz}, \mathrm{H}_{\mathrm{d}}\right), 9.04\left(\mathrm{~s}, 1 \mathrm{H}, \mathrm{H}_{2}\right), 7.71\left(\mathrm{~d}, 1 \mathrm{H},{ }^{4} \mathrm{~J}_{\mathrm{H} 5-\mathrm{H} 7}=1.1 \mathrm{~Hz}\right.$, $\left.\mathrm{H}_{5}\right), 7.37\left(\mathrm{~d}, 1 \mathrm{H},{ }^{4} \mathrm{JHz}^{\prime}-\mathrm{H6^{ \prime }}=2.1 \mathrm{~Hz}, \mathrm{H}_{2^{\prime}}\right), 7.23\left(\mathrm{~m}, 2 \mathrm{H}, \mathrm{H}_{6^{\prime}}, \mathrm{H}_{11^{\prime}}\right), 7.11\left(\mathrm{~m}, 2 \mathrm{H}, \mathrm{H}_{10^{\prime}}, \mathrm{H}_{\mathrm{g}}\right), 6.94(\mathrm{~d}, 1 \mathrm{H}$, $\left.{ }^{3} J_{\mathrm{H}^{\prime}-\mathrm{H}^{\prime}}=8.5 \mathrm{~Hz}, \mathrm{H}_{5^{\prime}}\right), 6.58\left(\mathrm{~d}, 1 \mathrm{H},{ }^{4} J_{\mathrm{H} 7-\mathrm{H} 5}=1.1 \mathrm{~Hz}, \mathrm{H}_{7}\right), 4.84\left(\mathrm{~d}, 2 \mathrm{H},{ }^{3} \mathrm{~J}_{\mathrm{He}-\mathrm{Hd}}=5.4 \mathrm{~Hz}, \mathrm{H}_{\mathrm{e}}\right), 4.47(\mathrm{~d}$, $\left.2 \mathrm{H},{ }^{3} J_{\mathrm{Hh}-\mathrm{Hg}}=5.6 \mathrm{~Hz}, \mathrm{H}_{\mathrm{h}}\right), 4.35\left(\mathrm{q}, 2 \mathrm{H},{ }^{3} \mathrm{~J}_{\mathrm{Hb}-\mathrm{Hc}}=7.1 \mathrm{~Hz}, \mathrm{H}_{\mathrm{b}}\right), 3.90\left(\mathrm{~s}, 3 \mathrm{H}, \mathrm{H}_{\mathrm{f}}\right), 3.88\left(\mathrm{~s}, 3 \mathrm{H}, \mathrm{H}_{\mathrm{i}}\right), 1.39(\mathrm{t}$, $\left.3 \mathrm{H},{ }^{3} \mathrm{~J}_{\mathrm{Hc}-\mathrm{Hb}}=7.1 \mathrm{~Hz}, \mathrm{H}_{\mathrm{c}}\right) ;{ }^{13} \mathrm{C} \mathrm{NMR}\left(100 \mathrm{MHz}, \mathrm{CDCl}_{3}\right) \delta 168.5\left(\mathrm{C}_{\mathrm{a}}\right), 156.3\left(\mathrm{C}_{4}\right), 154.9\left(\mathrm{C}_{4^{\prime}}\right), 153.2$ $\left(d,{ }^{1} J_{C^{\prime}-F}=240 \mathrm{~Hz}, C_{8^{\prime}}\right), 151.1\left(C_{2}\right), 145.6\left(d,{ }^{3} J_{C 12^{\prime}-F}=10 \mathrm{~Hz}, C_{12^{\prime}}\right), 145.1\left(C_{8}\right), 142.0\left(2 \mathrm{C}, C_{8 a}, C_{9^{\prime}}\right)$, $130.8\left(C_{1^{\prime}}\right), 129.4\left(C_{2^{\prime}}\right), 126.9\left(C_{6^{\prime}}\right), 123.1\left(C_{3^{\prime}}\right), 122.4\left(d,{ }^{3} J_{C 10^{\prime}-F}=4 \mathrm{~Hz}, C_{10^{\prime}}\right), 119.9(C N), 118.9$ $\left(d,{ }^{4} J_{C 11^{\prime}-F}=4 \mathrm{~Hz}, C_{11^{\prime}}\right), 118.6\left(C_{5}\right), 118.3\left(C_{4 a}\right), 112.5\left(C_{5^{\prime}}\right), 108.1\left(C_{6}\right), 106.7\left(C_{7}\right), 104.9\left(C_{3}\right), 61.3$ $\left(C_{b}\right), 56.4\left(C_{i}\right), 56.3\left(C_{f}\right), 51.7\left(C_{e}\right), 47.5\left(C_{h}\right), 14.3\left(C_{c}\right)$; HRMS (ESI) $m / z$ calculated for $\mathrm{C}_{28} \mathrm{H}_{26}{ }^{35} \mathrm{ClFN}_{5} \mathrm{O}_{4} \quad[\mathrm{M}+\mathrm{H}]^{+}:$550.1657, found $\mathrm{C}_{28} \mathrm{H}_{26}{ }^{35} \mathrm{ClFN}_{5} \mathrm{O}_{4} \quad[\mathrm{M}+\mathrm{H}]^{+}: 550.1661$ (100\%); $\mathrm{C}_{28} \mathrm{H}_{26}{ }^{37} \mathrm{CIFN}_{5} \mathrm{O}_{4}[\mathrm{M}+\mathrm{H}]^{+}: 552.1703$ (33\%).

\subsubsection{Ethyl 4-[(3-chloro-4-methoxybenzyl)amino]-6-cyano-8-[((6-fluoropyridin-2-yl) methyl)amino]quinoline-3-carboxylate (21d):}

The crude product $10 \mathrm{~b}(0.14 \mathrm{~g}$, synthesis in supporting information) was dissolved in dichloromethane $(30 \mathrm{~mL}$ ) before addition of a saturated aqueous sodium hydrogen carbonate solution (50 mL, $\mathrm{pH}$ 8-9). After decantation, the aqueous layer was extracted with dichloromethane $(7 \times 30 \mathrm{~mL})$. The combined organic layers were washed with brine $(40 \mathrm{~mL})$, dried over magnesium sulfate, filtered and concentrated under reduced pressure to obtain (6fluoropyridine-2-yl)methylamine ( $0.13 \mathrm{~g}, 1.27 \mathrm{mmol}, 3.0 \mathrm{eq}$.) as a yellow oil. This residue was dissolved, under argon atmosphere, in a mixture of 14 [28] (160 mg, $0.34 \mathrm{mmol}, 1.0$ eq.), palladium(II) acetate ( $7.6 \mathrm{mg}, 33.9 \mu \mathrm{mol}, 0.1 \mathrm{eq}$.), caesium carbonate $(549 \mathrm{mg}, 1.69 \mathrm{mmol}, 5.0$ eq.), and BINAP (42 mg, $67.5 \mu \mathrm{mol}, 0.2$ eq.) in anhydrous toluene $(15 \mathrm{~mL})$. The resulting mixture was refluxed for 4 hours in a sealed flask. After cooling to room temperature, the reaction mixture was filtered on Celite 545 and washed with dichloromethane $(210 \mathrm{~mL})$. The 
filtrate was concentrated under reduced pressure and the crude residue was purified by column chromatography $\left(\mathrm{SiO}_{2}\right.$, ethyl acetate/cyclohexane, 3/7, v/v) to give the desired product $21 \mathrm{~d}$ (163 $\mathrm{mg}, 0.31 \mathrm{mmol}, 93 \%)$ as an orange solid. $\mathrm{R}_{\mathrm{f}}\left(\mathrm{SiO}_{2}\right.$, cyclohexane/ethyl acetate, 7/3, v/v): 0.38; Mp: $152 \pm 1{ }^{\circ} \mathrm{C} ; \mathrm{IR}\left(\mathrm{cm}^{-1}\right)$ v $2229\left(\mathrm{v}_{\mathrm{C}=\mathrm{N}}\right), 1669\left(\mathrm{v}_{\mathrm{C}=\mathrm{O}}\right), 1594\left(\delta_{\mathrm{N}-\mathrm{H}}\right), 1531\left(\delta_{\mathrm{N}-\mathrm{H}}\right)$, $1501\left(v_{\mathrm{C}=\mathrm{C}}\right), 1434\left(\delta_{\mathrm{CH} 3}\right), 1252\left(\mathrm{v}_{\mathrm{C}-\mathrm{O}}\right), 1191\left(\mathrm{v}_{\mathrm{C}-\mathrm{O}}\right), 1064\left(\mathrm{v}_{\mathrm{C}-\mathrm{O}}\right), 1023\left(\mathrm{v}_{\mathrm{C}-\mathrm{F}}\right) ;{ }^{1} \mathrm{H}$ NMR $(400 \mathrm{MHz}$, $\left.\mathrm{CDCl}_{3}\right) \delta 9.61$ (brs, $\left.1 \mathrm{H}, \mathrm{H}_{\mathrm{d}}\right), 9.05\left(\mathrm{~s}, 1 \mathrm{H}, \mathrm{H}_{2}\right), 7.73\left(\mathrm{~m}, 2 \mathrm{H}, \mathrm{H}_{5}, \mathrm{H}_{10^{\prime}}\right), 7.38\left(\mathrm{~d}, 1 \mathrm{H},{ }^{4} \mathrm{JH}_{\mathrm{Hz}}-\mathrm{H}^{\prime}=2.2 \mathrm{~Hz}\right.$, $\left.\mathrm{H}_{2^{\prime}}\right), 7.23\left(\mathrm{~m}, 3 \mathrm{H}, \mathrm{H}_{6^{\prime}}, \mathrm{H}_{11^{\prime}}, \mathrm{H}_{\mathrm{g}}\right), 6.94\left(\mathrm{~d}, 1 \mathrm{H},{ }^{3} \mathrm{H}_{\mathrm{H}^{\prime}-\mathrm{H} 6^{\prime}}=8.5 \mathrm{~Hz}, \mathrm{H}_{5^{\prime}}\right), 6.84\left(\mathrm{~m}, 1 \mathrm{H}, \mathrm{H}_{9^{\prime}}\right), 6.59(\mathrm{~d}, 1 \mathrm{H}$, $\left.{ }^{4} J_{\mathrm{H} 7-\mathrm{H} 5}=1.1 \mathrm{~Hz}, \mathrm{H}_{7}\right), 4.85\left(\mathrm{~d}, 2 \mathrm{H},{ }^{3} \mathrm{~J}_{\mathrm{He}-\mathrm{Hd}}=5.4 \mathrm{~Hz}, \mathrm{He}_{\mathrm{e}}\right), 4.57\left(\mathrm{~d}, 2 \mathrm{H},{ }^{3} \mathrm{~J}_{\mathrm{Hh}-\mathrm{Hg}}=6.0 \mathrm{~Hz}, \mathrm{H}_{\mathrm{h}}\right), 4.36(\mathrm{q}$, $\left.2 \mathrm{H},{ }^{3} J_{\mathrm{Hb}-\mathrm{Hc}}=7.1 \mathrm{~Hz}, \mathrm{H}_{\mathrm{b}}\right), 3.90\left(\mathrm{~s}, 3 \mathrm{H}, \mathrm{H}_{\mathrm{f}}\right), 1.39\left(\mathrm{t}, 3 \mathrm{H},{ }^{3} \mathrm{~J}_{\mathrm{Hc}-\mathrm{Hb}}=7.1 \mathrm{~Hz}, \mathrm{H}_{\mathrm{c}}\right) ;{ }^{13} \mathrm{C} \mathrm{NMR}(100 \mathrm{MHz}$, $\left.\mathrm{CDCl}_{3}\right) \delta 168.4\left(\mathrm{C}_{\mathrm{a}}\right), 163.5\left(\mathrm{~d},{ }^{1} \mathrm{~J}_{\mathrm{C} 8^{\prime}-\mathrm{F}}=239 \mathrm{~Hz}, \mathrm{C}_{8^{\prime}}\right), 157.1\left(\mathrm{~d},{ }^{3} \mathrm{~J}_{\mathrm{C} 12^{\prime}-\mathrm{F}}=13 \mathrm{~Hz}, \mathrm{C}_{12^{\prime}}\right), 156.4\left(\mathrm{C}_{4}\right), 155.0$ $\left(C_{4^{\prime}}\right), 150.9\left(C_{2}\right), 144.9\left(C_{8}\right), 141.9\left(d,{ }^{3} J_{C 10^{\prime}-F}=8 \mathrm{~Hz}, C_{10^{\prime}}\right), 141.8\left(C_{8 a}\right), 130.6\left(C_{1^{\prime}}\right), 129.4\left(C_{2^{\prime}}\right)$, $126.9\left(C_{6^{\prime}}\right), 123.1\left(C_{3^{\prime}}\right), 119.8(C N), 118.7\left(2 C, C_{5}, C_{3}\right), 118.3\left(d,{ }^{4} J_{C 11^{\prime}-F}=4 \mathrm{~Hz}, C_{11^{\prime}}\right), 112.6\left(C_{5^{\prime}}\right)$, $108.3\left(C_{6}\right), 108.2\left(d,{ }^{2} J_{C^{\prime}-F}=37 \mathrm{~Hz}, C_{9^{\prime}}\right), 106.9\left(C_{7}\right), 104.9\left(C_{4 a}\right), 61.3\left(C_{b}\right), 56.3\left(C_{f}\right), 51.7\left(C_{e}\right)$, $48.2\left(\mathrm{C}_{h}\right), 14.3\left(\mathrm{C}_{\mathrm{c}}\right)$; HRMS (ESI) $\mathrm{m} / \mathrm{z}$ calculated for $\mathrm{C}_{27} \mathrm{H}_{24}{ }^{35} \mathrm{ClFN}_{5} \mathrm{O}_{3}[\mathrm{M}+\mathrm{H}]^{+}: 520.1552$, found $\mathrm{C}_{27} \mathrm{H}_{24}{ }^{35} \mathrm{CIFN}_{5} \mathrm{O}_{3}[\mathrm{M}+\mathrm{H}]^{+}: 520.1584$ (100\%); $\mathrm{C}_{27} \mathrm{H}_{24}{ }^{37} \mathrm{CIFN}_{5} \mathrm{O}_{3}[\mathrm{M}+\mathrm{H}]^{+}: 522.1701$ (33\%).

\subsubsection{General procedure for 6-9:}

A $1.1 \mathrm{M}$ solution of lithium tri-tert-butoxyaluminum hydride in tetrahydrofuran $(0.99 \mathrm{~mL}, 1.1$ mmol, 6.0 eq. for 6, 7 and 8, 10.0 eq. for 9) was added, under stirring and argon atmosphere, to a solution of 21a-d (1.0 eq.) in anhydrous tetrahydrofuran $(15 \mathrm{~mL})$. The resulting mixture was stirred at $60^{\circ} \mathrm{C}$ for 20 hours. After cooling to room temperature, water $(20 \mathrm{~mL})$ was added under stirring. The aqueous layer was extracted with ethyl acetate $(3 \times 40 \mathrm{~mL})$, and dichloromethane $(3 \times 30 \mathrm{~mL})$. These two organic layers were separately washed with brine $(100 \mathrm{~mL})$. The combined organic layers were dried over sodium sulfate, filtered and concentrated under reduced pressure. The crude product was purified by column chromatography $\left(\mathrm{SiO}_{2}\right.$, ethanol/dichloromethane, 5/95, v/v) to give the desired products.

\subsection{4-[(3-Chloro-4-methoxybenzyl)amino]-8-(4-fluoropiperidin-1-yl)-3- (hydroxymethyl)quinoline-6-carbonitrile (6):}

Yellow solid; yield 31\% (25 mg, $55.0 \mu \mathrm{mol}) ; \mathrm{R}_{\mathrm{f}}\left(\mathrm{SiO}_{2}\right.$, ethanol/dichloromethane, 5/95, v/v): 0.14; Mp: $215 \pm 1^{\circ} \mathrm{C} ; \mathrm{IR}\left(\mathrm{cm}^{-1}\right) \vee 2226\left(\mathrm{v}_{\mathrm{C} \equiv \mathrm{N}}\right), 1504\left(\delta_{\mathrm{N}-\mathrm{H}}\right), 1256\left(\mathrm{v}_{\mathrm{C}-\mathrm{O}}\right), 1064\left(\mathrm{v}_{\mathrm{C}-\mathrm{O}}\right), 1015\left(\mathrm{v}_{\mathrm{C}-\mathrm{F}}\right) ;{ }^{1} \mathrm{H} \mathrm{NMR}$ $\left(400 \mathrm{MHz}, \mathrm{DMSO}-\mathrm{d}_{6}\right) \delta 8.47\left(\mathrm{~m}, 1 \mathrm{H}, \mathrm{H}_{5}\right), 8.43\left(\mathrm{~s}, 1 \mathrm{H}, \mathrm{H}_{2}\right), 7.42(\mathrm{~m}, 1 \mathrm{H}, \mathrm{NH}), 7.39\left(\mathrm{~d}, 1 \mathrm{H},{ }^{4} \mathrm{JH}_{\mathrm{H}^{\prime}-\mathrm{H} 6^{\prime}}\right.$ $\left.=2.1 \mathrm{~Hz}, \mathrm{H}_{2^{\prime}}\right), 7.27\left(\mathrm{~m}, 1 \mathrm{H}, \mathrm{H}_{7}\right), 7.23\left(\mathrm{dd}, 1 \mathrm{H},{ }^{3} \mathrm{JH}_{\mathrm{G}^{\prime}-\mathrm{H} 5^{\prime}}=8.5 \mathrm{~Hz},{ }^{4} \mathrm{H}_{6^{\prime}-\mathrm{H} 2^{\prime}}=2.1 \mathrm{~Hz}, \mathrm{H}_{6^{\prime}}\right), 7.10(\mathrm{~d}, 1 \mathrm{H}$, $\left.{ }^{3} \mathrm{JH}_{\mathrm{H}^{\prime}-\mathrm{H} 6^{\prime}}=8.5 \mathrm{~Hz}, \mathrm{H}_{5^{\prime}}\right), 5.41\left(\mathrm{t}, 1 \mathrm{H},{ }^{3} \mathrm{~J}_{\mathrm{OH}-\mathrm{Ha}}=4.9 \mathrm{~Hz}, \mathrm{OH}\right), 4.88\left(\mathrm{~m}, 1 \mathrm{H}, \mathrm{H}_{\mathrm{f}}\right), 4.79\left(\mathrm{~d}, 2 \mathrm{H},{ }^{3} \mathrm{~J}_{\mathrm{Hb}-\mathrm{NH}}=6.7\right.$ $\left.\mathrm{Hz}, \mathrm{H}_{\mathrm{b}}\right), 4.44\left(\mathrm{~d}, 2 \mathrm{H}, 3^{3} \mathrm{Ha}_{\mathrm{H}-\mathrm{OH}}=4.9 \mathrm{~Hz}, \mathrm{H}_{\mathrm{a}}\right), 3.81\left(\mathrm{~s}, 3 \mathrm{H}, \mathrm{H}_{\mathrm{c}}\right), 3.35\left(\mathrm{~m}, 4 \mathrm{H}, \mathrm{H}_{\mathrm{d}}, \mathrm{H}_{\mathrm{d}^{\prime}}\right), 2.02\left(\mathrm{~m}, 4 \mathrm{H}, \mathrm{H}_{\mathrm{e}}\right.$ $\left.\mathrm{H}_{\mathrm{e}^{\prime}}\right) ;{ }^{13} \mathrm{C}$ NMR (100 MHz, DMSO-d 6 ) $\delta 153.4\left(\mathrm{C}_{4^{\prime}}\right), 153.1\left(\mathrm{C}_{2}\right), 150.4\left(\mathrm{C}_{4}\right), 149.8\left(\mathrm{C}_{8}\right), 143.9\left(\mathrm{C}_{8 \mathrm{a}}\right)$, $134.1\left(C_{1^{\prime}}\right), 127.8\left(C_{2^{\prime}}\right), 126.2\left(C_{6^{\prime}}\right), 122.0\left(C_{5}\right), 121.0\left(C_{3^{\prime}}\right), 120.7\left(C_{4 a}\right), 119.7(C N), 115.8\left(C_{7}\right)$, $115.7\left(C_{3}\right), 112.9\left(C_{5^{\prime}}\right), 106.6\left(C_{6}\right), 88.5\left(d,{ }^{1} J_{C f-F}=168 \mathrm{~Hz}, C_{f}\right), 59.6\left(C_{a}\right), 56.1\left(C_{c}\right), 47.6\left(C_{b}\right), 47.5$ $\left(2 \mathrm{C}, \mathrm{C}_{d}, \mathrm{C}_{\mathrm{d}^{\prime}}\right), 31.3\left(\mathrm{~d}, 2 \mathrm{C},{ }^{2} \mathrm{~J}_{\mathrm{Ce} / \mathrm{e}^{\prime}-\mathrm{F}}=19 \mathrm{~Hz}, \mathrm{C}_{\mathrm{e}}, \mathrm{C}_{\mathrm{e}^{\prime}}\right)$; HRMS (ESI) $\mathrm{m} / \mathrm{z}$ calculated for $\mathrm{C}_{24} \mathrm{H}_{25}{ }^{35} \mathrm{ClFN}_{4} \mathrm{O}_{2}$ $[\mathrm{M}+\mathrm{H}]^{+}: 455.1650$, found $\mathrm{C}_{24} \mathrm{H}_{25}{ }^{35} \mathrm{ClFN}_{4} \mathrm{O}_{2}[\mathrm{M}+\mathrm{H}]^{+}: 455.1617(100 \%) ; \mathrm{C}_{24} \mathrm{H}_{25}{ }^{37} \mathrm{ClFN}_{4} \mathrm{O}_{2}[\mathrm{M}+\mathrm{H}]^{+}$: 457.1621 (33\%). 


\subsection{4-[(3-Chloro-4-methoxybenzyl)amino]-8-(3-fluoroazetidin-1-yl)-3- (hydroxymethyl)quinoline-6-carbonitrile (7):}

Yellow solid; yield 55\% (90 mg, $0.21 \mathrm{mmol}) ; \mathrm{R}_{\mathrm{f}}\left(\mathrm{SiO}_{2}\right.$, ethanol/dichloromethane, 5/95, v/v): 0.47; Mp: $162 \pm 1{ }^{\circ} \mathrm{C} ; \mathrm{IR}\left(\mathrm{cm}^{-1}\right)$ v $3396\left(\mathrm{v}_{\mathrm{N}-\mathrm{H}}\right), 2228\left(\mathrm{v}_{\mathrm{C}=\mathrm{N}}\right), 1503\left(\delta_{\mathrm{N}-\mathrm{H}}\right), 1281\left(\mathrm{v}_{\mathrm{C}-\mathrm{O}}\right), 1260\left(\mathrm{v}_{\mathrm{C}-\mathrm{O}}\right)$, 1065 (vc-o); ${ }^{1} \mathrm{H}$ NMR (400 MHz, DMSO-d 6 ) $\delta 8.33\left(\mathrm{~s}, 1 \mathrm{H}, \mathrm{H}_{2}\right), 8.14\left(\mathrm{~d}, 1 \mathrm{H},{ }^{4} J_{\mathrm{H} 5-\mathrm{H} 7}=1.4 \mathrm{~Hz}, \mathrm{H}_{5}\right)$, $7.36\left(\mathrm{~d}, 1 \mathrm{H},{ }^{4} \mathrm{~J}_{\mathrm{Hz} 2^{\prime}-\mathrm{H} 6^{\prime}}=2.1 \mathrm{~Hz}, \mathrm{H}_{2^{\prime}}\right), 7.24(\mathrm{~m}, 1 \mathrm{H}, \mathrm{NH}), 7.21\left(\mathrm{dd}, 1 \mathrm{H},{ }^{4} J_{\mathrm{H} 6^{\prime}-\mathrm{H} 2^{\prime}}=2.1 \mathrm{~Hz},{ }^{3} \mathrm{JH}_{\mathrm{H}^{\prime}-\mathrm{H} 5^{\prime}}=8.4\right.$ $\left.\mathrm{Hz}, \mathrm{H}_{6^{\prime}}\right), 7.10\left(\mathrm{~d}, 1 \mathrm{H},{ }^{3} J_{\mathrm{H}^{\prime}-\mathrm{H} 6^{\prime}}=8.4 \mathrm{~Hz}, \mathrm{H}_{5^{\prime}}\right), 6.69\left(\mathrm{~d}, 1 \mathrm{H},{ }^{4} J_{\mathrm{H} 7-\mathrm{H} 5}=1.4 \mathrm{~Hz}, \mathrm{H}_{7}\right), 5.47\left(\mathrm{~m}, 1 \mathrm{H}, \mathrm{H}_{\mathrm{e}}\right), 5.36$ $\left(\mathrm{t}, 1 \mathrm{H},{ }^{3} \mathrm{~J}_{\mathrm{OH}-\mathrm{Ha}}=5.2 \mathrm{~Hz}, \mathrm{OH}\right), 4.76\left(\mathrm{~d}, 2 \mathrm{H},{ }^{3} \mathrm{~J}_{\mathrm{Hb}-\mathrm{NH}}=6.7 \mathrm{~Hz}, \mathrm{H}_{\mathrm{b}}\right), 4.54\left(\mathrm{~m}, 2 \mathrm{H}, \mathrm{H}_{\mathrm{d}}\right.$ or $\left.\mathrm{H}_{\mathrm{d}^{\prime}}\right), 4.41(\mathrm{~d}, 2 \mathrm{H}$, $\left.{ }^{3} \mathrm{~J} \mathrm{Ha}_{\mathrm{OH}}=5.2 \mathrm{~Hz}, \mathrm{H}_{\mathrm{a}}\right), 4.25\left(\mathrm{~m}, 2 \mathrm{H}, \mathrm{H}_{\mathrm{d}}\right.$ or $\left.\mathrm{H}_{\mathrm{d}^{\prime}}\right), 3.81\left(\mathrm{~s}, 3 \mathrm{H}, \mathrm{H}_{\mathrm{c}}\right) ;{ }^{13} \mathrm{C} \mathrm{NMR}\left(100 \mathrm{MHz}, \mathrm{DMSO}-\mathrm{d}_{6}\right) \delta$ $153.4\left(C_{4^{\prime}}\right), 152.8\left(C_{2}\right), 149.4\left(C_{4}\right), 147.8\left(C_{8}\right), 141.6\left(C_{8 a}\right), 134.3\left(C_{1^{\prime}}\right), 127.8\left(C_{2^{\prime}}\right), 126.1\left(C_{6^{\prime}}\right)$, $121.0\left(C_{3^{\prime}}\right), 120.4\left(C_{4 a}\right), 119.9(C N), 117.2\left(C_{5}\right), 115.9\left(C_{3}\right), 112.9\left(C_{5^{\prime}}\right), 108.1\left(C_{7}\right), 106.9\left(C_{6}\right), 84.3$ $\left(d,{ }^{1} J_{\text {Ce-F }}=196 \mathrm{~Hz}, C_{e}\right), 61.7\left(d, 2 C^{2}{ }^{2} C_{C d / d^{\prime}-F}=5 \mathrm{~Hz}, C_{d} / d^{\prime}\right), 59.7\left(C_{a}\right), 56.0\left(C_{c}\right), 47.5\left(C_{b}\right) ;$ HRMS (ESI) $\mathrm{m} / \mathrm{z}$ calculated for $\mathrm{C}_{22} \mathrm{H}_{21}{ }^{35} \mathrm{ClFN}_{4} \mathrm{O}_{2}[\mathrm{M}+\mathrm{H}]^{+}: 427.1337$, found $\mathrm{C}_{22} \mathrm{H}_{21}{ }^{35} \mathrm{ClFN}_{4} \mathrm{O}_{2}[\mathrm{M}+\mathrm{H}]^{+}: 427.1350$ (100\%); $\mathrm{C}_{22} \mathrm{H}_{21}{ }^{37} \mathrm{ClFN}_{4} \mathrm{O}_{2}[\mathrm{M}+\mathrm{H}]^{+}: 429.1253$ (33\%).

\subsection{4-[(3-chloro-4-methoxybenzyl)amino]-8-[((6-fluoro-5-methoxypyridin-2- yl)methyl)amino]-3-(hydroxymethyl)quinoline-6-carbonitrile (8):}

Light yellow solid; yield 55\% (80 mg, $0.16 \mathrm{mmol}) ; \mathrm{R}_{\mathrm{f}}\left(\mathrm{SiO}_{2}\right.$, ethanol/dichloromethane, 5/95, v/v): 0.40; Mp: $194 \pm 1^{\circ} \mathrm{C} ; \mathrm{IR}\left(\mathrm{cm}^{-1}\right)$ v $3409\left(\mathrm{v}_{\mathrm{N}-\mathrm{H}}\right), 2227\left(\mathrm{v}_{\mathrm{C}=\mathrm{N}}\right), 1546\left(\delta_{\mathrm{N}-\mathrm{H}}\right), 1260\left(\mathrm{v}_{\mathrm{C}-\mathrm{O}}\right), 1004\left(\mathrm{v}_{\mathrm{C}-}\right.$ F); ${ }^{1} \mathrm{H} N M R\left(400 \mathrm{MHz}, \mathrm{DMSO}-\mathrm{d}_{6}\right) \delta 8.38\left(\mathrm{~s}, 1 \mathrm{H}, \mathrm{H}_{2}\right), 8.01\left(\mathrm{~d}, 1 \mathrm{H},{ }^{4} \mathrm{H}_{\mathrm{H} 5-\mathrm{H} 7}=1.2 \mathrm{~Hz}, \mathrm{H}_{5}\right), 7.59$ (dd, $\left.1 \mathrm{H},{ }^{4} J_{\mathrm{H} 10^{\prime}-\mathrm{F}}=10.6 \mathrm{~Hz},{ }^{3} \mathrm{H}_{\mathrm{H} 10^{\prime}-\mathrm{H} 11^{\prime}}=8.2 \mathrm{~Hz}, \mathrm{H}_{10^{\prime}}\right), 7.45\left(\mathrm{t}, 1 \mathrm{H},{ }^{3} J_{\mathrm{He}-\mathrm{Hf}}=6.3 \mathrm{~Hz}, \mathrm{He}_{\mathrm{e}}\right), 7.37\left(\mathrm{~d}, 1 \mathrm{H},{ }^{4} J_{\mathrm{Hz}^{\prime}-}\right.$ $\left.\mathrm{H}^{\prime}=2.1 \mathrm{~Hz}, \mathrm{H}_{2^{\prime}}\right), 7.33\left(\mathrm{t}, 1 \mathrm{H}, 3^{3} \mathrm{Hb}_{\mathrm{Hb}}=6.8 \mathrm{~Hz}, \mathrm{H}_{\mathrm{b}}\right), 7.25\left(\mathrm{~d}, 1 \mathrm{H},{ }^{3} \mathrm{~J}_{\mathrm{H} 11^{\prime}-\mathrm{H} 10^{\prime}}=8.2 \mathrm{~Hz}, \mathrm{H}_{11^{\prime}}\right), 7.21$ (dd, $\left.1 \mathrm{H},{ }^{3} J_{\mathrm{H}^{\prime}-\mathrm{H} 5^{\prime}}=8.5 \mathrm{~Hz},{ }^{4} J_{\mathrm{H}^{\prime}-\mathrm{H} 2^{\prime}}=2.1 \mathrm{~Hz}, \mathrm{H}_{6^{\prime}}\right), 7.10\left(\mathrm{~d}, 1 \mathrm{H},{ }^{3} J_{\mathrm{H} 5^{\prime}-\mathrm{H} 6^{\prime}}=8.5 \mathrm{~Hz}, \mathrm{H}_{5^{\prime}}\right), 6.65\left(\mathrm{~d}, 1 \mathrm{H},{ }^{4} J_{\mathrm{H} 7-\mathrm{H} 5}\right.$ $\left.=1.2 \mathrm{~Hz}, \mathrm{H}_{7}\right), 5.39\left(\mathrm{t}, 1 \mathrm{H},{ }^{3} \mathrm{~J}_{\mathrm{OH}-\mathrm{Ha}}=5.1 \mathrm{~Hz}, \mathrm{OH}\right), 4.79\left(\mathrm{~d}, 2 \mathrm{H},{ }^{3} \mathrm{~J}_{\mathrm{Hc}-\mathrm{Hb}}=6.8 \mathrm{~Hz}, \mathrm{H}_{\mathrm{c}}\right), 4.49\left(\mathrm{~d}, 2 \mathrm{H},{ }^{3} \mathrm{~J}_{\mathrm{Hf}-}\right.$ $\left.\mathrm{He}=6.3 \mathrm{~Hz}, \mathrm{H}_{\mathrm{f}}\right), 4.44\left(\mathrm{~d}, 2 \mathrm{H},{ }^{3} \mathrm{~J}_{\mathrm{Ha}-\mathrm{OH}}=5.1 \mathrm{~Hz}, \mathrm{H}_{\mathrm{a}}\right), 3.84\left(\mathrm{~s}, 3 \mathrm{H}, \mathrm{H}_{\mathrm{g}}\right), 3.81\left(\mathrm{~s}, 3 \mathrm{H}, \mathrm{H}_{\mathrm{d}}\right) ;{ }^{13} \mathrm{C} \mathrm{NMR}(100$ $\left.\mathrm{MHz}, \mathrm{DMSO}-\mathrm{d}_{6}\right) \delta 153.4\left(\mathrm{C}_{4^{\prime}}\right), 152.8\left(\mathrm{C}_{2}\right), 151.9\left(\mathrm{~d},{ }^{1} \mathrm{~J}_{\mathrm{C8^{ \prime } - \mathrm { F }}}=235 \mathrm{~Hz}, \mathrm{C}_{8^{\prime}}\right), 149.9\left(\mathrm{C}_{4}\right), 146.1(\mathrm{~d}$, $\left.{ }^{3} J_{C 12^{\prime}-F}=11 \mathrm{~Hz}, C_{12^{\prime}}\right), 145.0\left(C_{3}\right), 140.8\left(d,{ }^{2} J_{C 9^{\prime}-F}=15 \mathrm{~Hz}, C_{9^{\prime}}\right), 139.3\left(C_{8 \mathrm{a}}\right), 134.3\left(C_{1^{\prime}}\right), 127.7\left(C_{2^{\prime}}\right)$, $126.1\left(C_{6^{\prime}}\right), 123.5$ (d, $\left.{ }^{3} J_{C 10^{\prime}-F}=3 \mathrm{~Hz}, C_{10^{\prime}}\right), 121.0\left(C_{3^{\prime}}\right), 120.3(C N), 119.7$ (d, $\left.{ }^{4} J_{C 11^{\prime}-F}=3 \mathrm{~Hz}, C_{11^{\prime}}\right)$, $119.2\left(C_{4 a}\right), 116.1\left(C_{3}\right), 114.8\left(C_{5}\right), 112.9\left(C_{5^{\prime}}\right), 107.2\left(C_{6}\right), 103.6\left(C_{7}\right), 59.7\left(C_{a}\right), 56.1\left(C_{g}\right), 56.0$ $\left(\mathrm{C}_{d}\right), 47.3\left(\mathrm{C}_{\mathrm{c}}\right), 46.2\left(\mathrm{C}_{\mathrm{f}}\right)$; HRMS (ESI) $\mathrm{m} / \mathrm{z}$ calculated for $\mathrm{C}_{26} \mathrm{H}_{24}{ }^{35} \mathrm{CIFN}_{5} \mathrm{O}_{3}[\mathrm{M}+\mathrm{H}]^{+}:$: 508.1552, found $\mathrm{C}_{26} \mathrm{H}_{24}{ }^{35} \mathrm{CIFN}_{5} \mathrm{O}_{3}[\mathrm{M}+\mathrm{H}]^{+}: 508.1528$ (100\%); $\mathrm{C}_{26} \mathrm{H}_{24}{ }^{37} \mathrm{CIFN}_{5} \mathrm{O}_{3}[\mathrm{M}+\mathrm{H}]^{+}: 510.1570$ (33\%).

\subsection{4-[(3-Chloro-4-methoxybenzyl)amino]-8-[((6-fluoropyridin-2-yl)methyl)amino]-} 3-(hydroxymethyl)quinoline-6-carbonitrile (9):

Light yellow solid; yield 41\% (15 mg, $31.4 \mu \mathrm{mol}) ; \mathrm{R}_{\mathrm{f}}\left(\mathrm{SiO}_{2}\right.$, ethanol/dichloromethane, $\left.1 / 9, \mathrm{v} / \mathrm{v}\right)$ : 0.40; Mp: $186 \pm 1{ }^{\circ} \mathrm{C} ; \mathrm{IR}\left(\mathrm{cm}^{-1}\right)$ v $3400\left(\mathrm{~V}_{\mathrm{N}-\mathrm{H}}\right), 2232\left(\mathrm{v}_{\mathrm{C} \equiv \mathrm{N}}\right), 1550\left(\delta_{\mathrm{N}-\mathrm{H}}\right), 1504\left(\delta_{\mathrm{N}-\mathrm{H}}\right), 1436\left(\delta_{\mathrm{CH} 3}\right)$, 1261 ( $\left.v_{\mathrm{c}-\mathrm{o}}\right), 1228\left(\mathrm{v}_{\mathrm{c}-\mathrm{o}}\right) ;{ }^{1} \mathrm{H}$ NMR (400 MHz, DMSO-d 6 ) $\delta 8.40\left(\mathrm{~s}, 1 \mathrm{H}, \mathrm{H}_{2}\right), 8.03\left(\mathrm{~m}, 1 \mathrm{H}, \mathrm{H}_{5}\right), 7.93$ $\left(\mathrm{m}, 1 \mathrm{H}, \mathrm{H}_{10^{\prime}}\right), 7.55\left(\mathrm{t}, 1 \mathrm{H},{ }^{3} \mathrm{~J}_{\mathrm{He}-\mathrm{Hf}}=6.2 \mathrm{~Hz}, \mathrm{H}_{\mathrm{e}}\right), 7.38\left(\mathrm{~d}, 1 \mathrm{H},{ }^{4} \mathrm{JH}_{\mathrm{H}^{\prime}-\mathrm{H} 6^{\prime}}=2.1 \mathrm{~Hz}, \mathrm{H}_{2^{\prime}}\right), 7.35\left(\mathrm{t}, 1 \mathrm{H},{ }^{3} \mathrm{~J}_{\mathrm{Hb}-}\right.$ $\left.\mathrm{Hc}=6.7 \mathrm{~Hz}, \mathrm{H}_{\mathrm{b}}\right), 7.29\left(\mathrm{~m}, 1 \mathrm{H}, \mathrm{H}_{11^{\prime}}\right), 7.23\left(\mathrm{dd}, 1 \mathrm{H},{ }^{3} \mathrm{JH}_{6^{\prime}-\mathrm{H} 5^{\prime}}=8.6 \mathrm{~Hz},{ }^{4} \mathrm{JH}^{\prime}-\mathrm{H2^{ \prime }}=2.1 \mathrm{~Hz}, \mathrm{H}_{6^{\prime}}\right), 7.10(\mathrm{~d}$, 
$\left.1 \mathrm{H},{ }^{3} J_{\mathrm{H}^{\prime}-\mathrm{H} 6^{\prime}}=8.6 \mathrm{~Hz}, \mathrm{H}_{5^{\prime}}\right), 7.05\left(\mathrm{~m}, 1 \mathrm{H}, \mathrm{H}_{9^{\prime}}\right), 6.66\left(\mathrm{~d}, 1 \mathrm{H},{ }^{4} \mathrm{~J}_{\mathrm{H} 7-\mathrm{H} 5}=1.2 \mathrm{~Hz}, \mathrm{H}_{7}\right), 5.40$ (brs, $\left.1 \mathrm{H}, \mathrm{OH}\right)$, $4.80\left(\mathrm{~d}, 2 \mathrm{H},{ }^{3} \mathrm{~J} \mathrm{Hc}-\mathrm{Hb}=6.7 \mathrm{~Hz}, \mathrm{H}_{\mathrm{c}}\right), 4.59\left(\mathrm{~d}, 2 \mathrm{H},{ }^{3} \mathrm{~J}_{\mathrm{Hf}-\mathrm{He}}=6.2 \mathrm{~Hz}, \mathrm{H}_{\mathrm{f}}\right), 4.45\left(\mathrm{~d}, 2 \mathrm{H},{ }^{3} \mathrm{~J}_{\mathrm{Ha}-\mathrm{OH}}=3.7 \mathrm{~Hz}, \mathrm{H}_{\mathrm{a}}\right)$, $3.81\left(\mathrm{~s}, 3 \mathrm{H}, \mathrm{H}_{\mathrm{d}}\right) ;{ }^{13} \mathrm{C}$ NMR (100 MHz, DMSO-d $\left.\mathrm{d}_{6}\right) \delta 162.6\left(\mathrm{~d},{ }^{1} \mathrm{~J}_{\mathrm{C} 8^{\prime}-\mathrm{F}}=235 \mathrm{~Hz}, \mathrm{C}_{8^{\prime}}\right), 158.0\left(\mathrm{~d},{ }^{3} \mathrm{~J}_{\mathrm{C} 12^{\prime}-\mathrm{F}}\right.$ $\left.=13 \mathrm{~Hz}, \mathrm{C}_{12^{\prime}}\right), 153.4\left(\mathrm{C}_{4^{\prime}}\right), 152.8\left(\mathrm{C}_{2}\right), 150.0\left(\mathrm{C}_{4}\right), 145.0\left(\mathrm{C}_{8}\right), 142.7\left(\mathrm{~d},{ }^{3} \mathrm{~J}_{\mathrm{C} 10^{\prime}-\mathrm{F}}=8 \mathrm{~Hz}, \mathrm{C}_{10^{\prime}}\right), 139.3$ $\left(C_{8 \mathrm{a}}\right), 134.3\left(\mathrm{C}_{1^{\prime}}\right), 127.8\left(\mathrm{C}_{2^{\prime}}\right), 126.1\left(\mathrm{C}_{6^{\prime}}\right), 121.0\left(\mathrm{C}_{3^{\prime}}\right), 120.2(\mathrm{CN}), 119.2\left(\mathrm{C}_{4 \mathrm{a}}\right), 118.9\left(\mathrm{~d},{ }^{4} \mathrm{~J}_{\mathrm{C} 11^{\prime}-\mathrm{F}}=3\right.$ $\left.\mathrm{Hz}, \mathrm{C}_{11^{\prime}}\right), 116.1\left(\mathrm{C}_{3}\right), 115.0\left(\mathrm{C}_{5}\right), 112.9\left(\mathrm{C}_{5^{\prime}}\right), 107.6\left(\mathrm{~d},{ }^{2} \mathrm{~J}_{\mathrm{Cg}^{\prime}-\mathrm{F}}=37 \mathrm{~Hz}, \mathrm{C}_{9^{\prime}}\right), 107.3\left(\mathrm{C}_{6}\right), 103.7\left(\mathrm{C}_{7}\right)$, $59.8\left(\mathrm{C}_{\mathrm{a}}\right), 56.0\left(\mathrm{C}_{\mathrm{d}}\right), 47.4\left(\mathrm{C}_{\mathrm{c}}\right), 46.9\left(\mathrm{C}_{\mathrm{f}}\right)$; HRMS (ESI) $\mathrm{m} / \mathrm{z}$ calculated for $\mathrm{C}_{25} \mathrm{H}_{22}{ }^{35} \mathrm{ClFN}_{5} \mathrm{O}_{2}[\mathrm{M}+\mathrm{H}]^{+}$: 478.1446, found $\mathrm{C}_{25} \mathrm{H}_{22}{ }^{35} \mathrm{CIFN}_{5} \mathrm{O}_{2}[\mathrm{M}+\mathrm{H}]^{+}: 478.1437$ (100\%); $\mathrm{C}_{25} \mathrm{H}_{22}{ }^{37} \mathrm{ClFN}_{5} \mathrm{O}_{2}[\mathrm{M}+\mathrm{H}]^{+}: 480.1476$ (33\%).

\subsubsection{Preparation of precursors for radiolabelling}

\subsection{8-Bromo-4-[(3-chloro-4-methoxybenzyl)amino]-3-(hydroxymethyl)quinoline-6- carbonitrile (22):}

A 1.1 $\mathrm{M}$ solution of lithium tri-tert-butoxyaluminum hydride in tetrahydrofuran $(5.70 \mathrm{~mL}, 6.27$ mmol, 6.0 eq.) was added, under stirring and argon atmosphere, to a solution of 14 [28] (0.5 $\mathrm{g}, 1.05 \mathrm{mmol}, 1.0$ eq.) in anhydrous tetrahydrofuran $(30 \mathrm{~mL})$. The resulting mixture was stirred at $60{ }^{\circ} \mathrm{C}$ for 20 hours. After cooling to room temperature, water $(20 \mathrm{~mL})$ was added under stirring. The aqueous layer was extracted with ethyl acetate $(3 \times 30 \mathrm{~mL})$ and dichloromethane $(3 \times 30 \mathrm{~mL})$. These two organic layers were separately washed with brine $(100 \mathrm{~mL})$, then combined, dried over magnesium sulfate, filtered and concentrated under reduced pressure. The crude residue was purified by column chromatography $\left(\mathrm{SiO}_{2}\right.$, ethanol/dichloromethane, $5 / 95, \mathrm{v} / \mathrm{v})$ to give the desired product $22(0.31 \mathrm{~g}, 0.72 \mathrm{mmol}, 69 \%)$ as a white solid. $\mathrm{R}_{\mathrm{f}}\left(\mathrm{SiO}_{2}\right.$, ethanol/dichloromethane, 5/95, v/v): 0.41; Mp: $209 \pm 1{ }^{\circ} \mathrm{C}$; IR $\left(\mathrm{cm}^{-1}\right) \vee 3403\left(\mathrm{v}_{\mathrm{N}-\mathrm{H}}\right), 2229\left(\mathrm{v}_{\mathrm{C} \equiv \mathrm{N}}\right)$, $1505\left(\delta_{\mathrm{N}-\mathrm{H}}\right), 1261$ ( $\left.\mathrm{v}_{\mathrm{C}-\mathrm{O}}\right), 1015\left(\mathrm{v}_{\mathrm{c}-\mathrm{O}}\right) ;{ }^{1} \mathrm{H}$ NMR $\left(400 \mathrm{MHz}, \mathrm{DMSO}-\mathrm{d}_{6}\right) \delta 8.96\left(\mathrm{~d}, 1 \mathrm{H},{ }^{4} \mathrm{~J}_{\mathrm{H} 5-\mathrm{H} 7}=1.5\right.$ $\left.\mathrm{Hz}, \mathrm{H}_{5}\right), 8.53\left(\mathrm{~s}, 1 \mathrm{H}, \mathrm{H}_{2}\right), 8.35\left(\mathrm{~d}, 1 \mathrm{H},{ }^{4} \mathrm{~J}_{\mathrm{H} 7-\mathrm{H} 5}=1.5 \mathrm{~Hz}, \mathrm{H}_{7}\right), 7.67\left(\mathrm{t}, 1 \mathrm{H},{ }^{3} J_{\mathrm{NH}-\mathrm{Hb}}=6.7 \mathrm{~Hz}, \mathrm{NH}\right), 7.40$ (d, $\left.1 \mathrm{H},{ }^{4} J_{\mathrm{H}^{\prime}-\mathrm{H} 6^{\prime}}=2.1 \mathrm{~Hz}, \mathrm{H}_{2^{\prime}}\right), 7.24\left(\mathrm{dd}, 1 \mathrm{H},{ }^{4} \mathrm{~J}_{\mathrm{H} 6^{\prime}-\mathrm{H} 2^{\prime}}=2.1 \mathrm{~Hz},{ }^{3} J_{\mathrm{H}^{\prime}-\mathrm{H} 5^{\prime}}=8.5 \mathrm{~Hz}, \mathrm{H}_{6^{\prime}}\right), 7.10(\mathrm{~d}, 1 \mathrm{H}$, $\left.{ }^{3} \mathrm{JH}_{\mathrm{H}^{\prime}-\mathrm{H}^{\prime}}=8.5 \mathrm{~Hz}, \mathrm{H}_{5^{\prime}}\right), 5.48\left(\mathrm{t}, 1 \mathrm{H},{ }^{3} \mathrm{~J}_{\mathrm{OH}-\mathrm{Ha}}=5.2 \mathrm{~Hz}, \mathrm{OH}\right), 4.85\left(\mathrm{~d}, 2 \mathrm{H},{ }^{3} \mathrm{~J}_{\mathrm{Hb}-\mathrm{NH}}=6.7 \mathrm{~Hz}, \mathrm{Hb}_{\mathrm{b}}\right), 4.46(\mathrm{~d}$, $\left.2 \mathrm{H},{ }^{3} \mathrm{~J}_{\mathrm{Ha}-\mathrm{OH}}=5.2 \mathrm{~Hz}, \mathrm{H}_{\mathrm{a}}\right), 3.81\left(\mathrm{~s}, 3 \mathrm{H}, \mathrm{H}_{\mathrm{c}}\right) ;{ }^{13} \mathrm{C} \mathrm{NMR}\left(100 \mathrm{MHz}, \mathrm{DMSO}-\mathrm{d}_{6}\right) \delta 156.7\left(\mathrm{C}_{2}\right), 153.5\left(\mathrm{C}_{4^{\prime}}\right)$, $150.3\left(C_{4}\right), 146.8\left(C_{8 a}\right), 133.8\left(C_{1^{\prime}}\right), 132.6\left(C_{7}\right), 129.7\left(C_{5}\right), 127.9\left(C_{2^{\prime}}\right), 126.2\left(C_{6^{\prime}}\right), 125.5\left(C_{8}\right)$, $121.1\left(C_{3^{\prime}}\right), 120.4\left(C_{4 a}\right), 118.1(C N), 116.2\left(C_{3}\right), 113.0\left(C_{5^{\prime}}\right), 106.7\left(C_{6}\right), 59.6\left(C_{a}\right), 56.1\left(C_{c}\right), 47.5$ $\left(\mathrm{C}_{\mathrm{b}}\right)$; HRMS (ESI) $\mathrm{m} / \mathrm{z}$ calculated for $\mathrm{C}_{19} \mathrm{H}_{16}{ }^{35} \mathrm{Cl}^{79} \mathrm{BrN}_{3} \mathrm{O}_{2} \quad[\mathrm{M}+\mathrm{H}]^{+}:$432.0114, found $\mathrm{C}_{19} \mathrm{H}_{16}{ }^{35} \mathrm{Cl}^{79} \mathrm{BrN}_{3} \mathrm{O}_{2}[\mathrm{M}+\mathrm{H}]^{+}: 432.0105$ (77\%); $\mathrm{C}_{19} \mathrm{H}_{16}{ }^{37} \mathrm{Cl}^{79} \mathrm{BrN}_{3} \mathrm{O}_{2}[\mathrm{M}+\mathrm{H}]^{+}: 434.0087$ (100\%); $\mathrm{C}_{19} \mathrm{H}_{16}{ }^{37} \mathrm{Cl}^{81} \mathrm{BrN}_{3} \mathrm{O}_{2}[\mathrm{M}+\mathrm{H}]^{+}: 436.0092$ (24\%).

\subsection{8-Bromo-4-[(3-chloro-4-methoxybenzyl)amino]-3- [((triisopropylsilyl)oxy)methyl]quinoline-6-carbonitrile (23):}

Triisopropylsilyl chloride ( $74 \mu \mathrm{L}, 0.35 \mathrm{mmol}, 1.5$ eq.), triethylamine (64 $\mu \mathrm{L}, 0.46 \mathrm{mmol}, 2.0$ eq.), $\mathrm{N}, \mathrm{N}$-4-dimethylaminopyridine $(5.6 \mathrm{mg}, 45.8 \mu \mathrm{mol}, 0.2 \mathrm{eq}$.$) were added, under argon$ atmosphere and stirring, to a solution of $22(0.10 \mathrm{~g}, 0.25 \mathrm{mmol}, 1.0$ eq.) in anhydrous $\mathrm{N}, \mathrm{N}-$ dimethylformamide $(10 \mathrm{~mL})$. The resulting mixture was stirred at room temperature for 36 
hours before addition of water $(20 \mathrm{~mL})$. The solution was extracted with ethyl acetate $(3 \times 30$ $\mathrm{mL}$ ) and the combined organic layers were washed with brine $(3 \times 50 \mathrm{~mL})$, dried over anhydrous magnesium sulfate, filtered, and then concentrated under reduced pressure. The crude residue was purified by column chromatography $\left(\mathrm{SiO}_{2}\right.$, gradient: cyclohexane to ethyl acetate/cyclohexane, 2/8, v/v) to give the desired product $23(0.11 \mathrm{~g}, 0.19 \mathrm{mmol}, 76 \%)$ as a white solid. $\mathrm{R}_{\mathrm{f}}\left(\mathrm{SiO}_{2}\right.$, ethyl acetate/cyclohexane, 2/8, v/v): 0.33; $\mathrm{Mp}: 124 \pm 1{ }^{\circ} \mathrm{C} ; \mathrm{IR}\left(\mathrm{cm}^{-1}\right) \vee 3281$ $\left(v_{\mathrm{N}-\mathrm{H}}\right), 2943\left(\mathrm{v}_{\text {as CH3 }}\right), 2864\left(\mathrm{v}_{\mathrm{s}} \mathrm{CH} 3\right), 2227\left(\mathrm{v}_{\mathrm{C} \equiv \mathrm{N}}\right), 1503\left(\delta_{\mathrm{N}-\mathrm{H}}\right), 1256\left(\mathrm{v}_{\mathrm{C}-\mathrm{O}}\right), 1060\left(\mathrm{v}_{\mathrm{C}-\mathrm{O}}\right), 807\left(\mathrm{v}_{\mathrm{C}-\mathrm{Si}}\right) ;{ }^{1} \mathrm{H}$ $\operatorname{NMR}\left(200 \mathrm{MHz}, \mathrm{CDCl}_{3}\right) \delta 8.57\left(\mathrm{~s}, 1 \mathrm{H}, \mathrm{H}_{2}\right), 8.56\left(\mathrm{~s}, 1 \mathrm{H}, \mathrm{H}_{5}\right), 8.04\left(\mathrm{~s}, 1 \mathrm{H}, \mathrm{H}_{7}\right), 7.35\left(\mathrm{~d}, 1 \mathrm{H},{ }^{4} \mathrm{JH}_{\mathrm{H}^{\prime}-\mathrm{H} 6^{\prime}}\right.$ $\left.=1.8 \mathrm{~Hz}, \mathrm{H}_{2^{\prime}}\right), 7.17\left(\mathrm{dd}, 1 \mathrm{H}, 3^{3} \mathrm{H}_{6^{\prime}-\mathrm{H} 5^{\prime}}=8.3 \mathrm{~Hz},{ }^{4} J_{\mathrm{H} 6^{\prime}-\mathrm{H} 2^{\prime}}=1.8 \mathrm{~Hz}, \mathrm{H}_{6^{\prime}}\right), 6.90\left(\mathrm{~d}, 1 \mathrm{H}, 3^{3} \mathrm{H}_{5^{\prime}-\mathrm{H}^{\prime}}=8.3 \mathrm{~Hz}\right.$, $\left.\mathrm{H}_{5^{\prime}}\right), 6.59(\mathrm{brs}, 1 \mathrm{H}, \mathrm{NH}), 4.81\left(\mathrm{~s}, 2 \mathrm{H}, \mathrm{H}_{\mathrm{a}}\right), 4.77\left(\mathrm{~d}, 2 \mathrm{H},{ }^{3} \mathrm{~J}_{\mathrm{Hd}-\mathrm{NH}}=5.2 \mathrm{~Hz}, \mathrm{H}_{\mathrm{d}}\right), 3.89\left(\mathrm{~s}, 3 \mathrm{H}, \mathrm{H}_{\mathrm{e}}\right), 1.06$ $\left(\mathrm{m}, 3 \mathrm{H}, \mathrm{H}_{\mathrm{b}}\right), 0.98\left(\mathrm{~s}, 18 \mathrm{H}, \mathrm{H}_{\mathrm{c}}\right) ;{ }^{13} \mathrm{C} \mathrm{NMR}\left(50 \mathrm{MHz}, \mathrm{CDCl}_{3}\right) \delta 155.1\left(\mathrm{C}_{4^{\prime}}\right), 153.5\left(\mathrm{C}_{4}\right), 152.0\left(\mathrm{C}_{2}\right)$, $147.9\left(C_{8 \mathrm{a}}\right), 133.1\left(\mathrm{C}_{7}\right), 131.5\left(\mathrm{C}_{1^{\prime}}\right), 130.7\left(\mathrm{C}_{5}\right), 129.4\left(\mathrm{C}_{2^{\prime}}\right), 127.0\left(\mathrm{C}_{6^{\prime}}\right), 125.8\left(\mathrm{C}_{8}\right), 123.3\left(\mathrm{C}_{3^{\prime}}\right)$, $120.7\left(C_{4 a}\right), 119.1\left(C_{3}\right), 117.9(C N), 112.5\left(C_{5^{\prime}}\right), 107.7\left(C_{6}\right), 63.2\left(C_{a}\right), 56.3\left(C_{e}\right), 52.1\left(C_{d}\right), 17.9$ (6C, $\left.\mathrm{C}_{c}\right), 11.8\left(3 \mathrm{C}, \mathrm{C}_{\mathrm{b}}\right)$; HRMS (ESI) $\mathrm{m} / z$ calculated for $\mathrm{C}_{28} \mathrm{H}_{36}{ }^{79} \mathrm{Br}^{35} \mathrm{CIN}_{3} \mathrm{O}_{2} \mathrm{Si}[\mathrm{M}+\mathrm{H}]^{+}:$588.1449, found $\mathrm{C}_{28} \mathrm{H}_{36}{ }^{79} \mathrm{Br}^{35} \mathrm{CIN}_{3} \mathrm{O}_{2} \mathrm{Si}[\mathrm{M}+\mathrm{H}]^{+}: 588.1412$ (77\%); $\mathrm{C}_{28} \mathrm{H}_{36}{ }^{79} \mathrm{Br}^{37} \mathrm{ClN}_{3} \mathrm{O}_{2} \mathrm{Si}[\mathrm{M}+\mathrm{H}]^{+}: 590.1365$ (100\%); $\mathrm{C}_{28} \mathrm{H}_{36}{ }^{81} \mathrm{Br}^{37} \mathrm{ClN}_{3} \mathrm{O}_{2} \mathrm{Si}[\mathrm{M}+\mathrm{H}]^{+}: 592.1424$ (24\%).

\subsection{4-[(3-Chloro-4-methoxybenzyl)amino]-8-(3-hydroxyazetidin-1-yl)-3- [((triisopropylsilyl)oxy)methyl]quinoline-6-carbonitrile (24):}

A solution of tert-butyl 3-hydroxyazetidine-1-carboxylate $(0.60 \mathrm{~g}, 3.46 \mathrm{mmol}, 3.4$ eq.) in anhydrous chloromethane $(5.0 \mathrm{~mL})$ was placed in a flask equipped with a screw cap and trifluoroacetic acid $(2.5 \mathrm{~mL}$ ) was added under argon atmosphere. The resulting mixture was stirred at room temperature for one hour and afterwards evaporated under vacuum for 3 hours. The residue was dissolved in anhydrous toluene $(20 \mathrm{~mL})$ under argon atmosphere and caesium carbonate $(2.00 \mathrm{~g}, 6.14 \mathrm{mmol}, 6.0 \mathrm{eq}$.) was added. After stirring at room temperature for 15 minutes, 23 ( $0.60 \mathrm{~g}, 1.02 \mathrm{mmol}, 1.0$ eq.), palladium(II) acetate ( $46 \mathrm{mg}, 0.20 \mathrm{mmol}, 0.2$ eq.), and 2,2'-bis(diphenylphosphino)-1,1'-binaphthyl (BINAP) (127 mg, $0.20 \mathrm{mmol}, 0.2$ eq.) were added. The resulting mixture was refluxed for 3 hours. After cooling to room temperature, the reaction mixture was concentrated under reduced pressure and the crude residue was purified by column chromatography $\left(\mathrm{SiO}_{2}\right.$, ethyl acetate/cyclohexane, 5/5, v/v) to give the desired product $24(0.30 \mathrm{~g}, 0.52 \mathrm{mmol}, 51 \%)$ as an orange solid. $\mathrm{R}_{\mathrm{f}}\left(\mathrm{SiO}_{2}\right.$, cyclohexane/ethyl acetate, 5/5, v/v): 0.29; Mp: $105 \pm 1{ }^{\circ} \mathrm{C}$; IR $\left(\mathrm{cm}^{-1}\right)$ v $2943\left(\mathrm{~V}_{\text {as CH3 }}\right), 2865\left(\mathrm{~V}_{\mathrm{s}}\right.$ $\left.\mathrm{CH}_{3}\right), 2222\left(\mathrm{v}_{\mathrm{C} \equiv \mathrm{N}}\right), 1502\left(\delta_{\mathrm{N}-\mathrm{H}}\right), 1436\left(\delta_{\mathrm{CH} 3}\right), 1257\left(\mathrm{v}_{\mathrm{C}-\mathrm{O}}\right), 1064\left(\mathrm{v}_{\mathrm{C}-\mathrm{O}}\right), 809\left(\mathrm{v}_{\mathrm{C}-\mathrm{Si}}\right), 683\left(\mathrm{v}_{\mathrm{C}-\mathrm{Cl}}\right) ;{ }^{1} \mathrm{H}$ NMR $\left(200 \mathrm{MHz} \mathrm{CDCl}_{3}\right) \delta 8.35\left(\mathrm{~s}, 1 \mathrm{H}, \mathrm{H}_{2}\right), 7.80\left(\mathrm{~d}, 1 \mathrm{H},{ }^{4} J_{\mathrm{H} 5-\mathrm{H} 7}=1.4 \mathrm{~Hz}, \mathrm{H}_{5}\right), 7.34\left(\mathrm{~d}, 1 \mathrm{H},{ }^{4} J_{\mathrm{H}^{\prime}-\mathrm{H}^{\prime}}=2.1\right.$ $\left.\mathrm{Hz}, \mathrm{H}_{2^{\prime}}\right), 7.16\left(\mathrm{dd}, 1 \mathrm{H},{ }^{3} \mathrm{JH}^{\prime}-\mathrm{H} 5^{\prime}=8.4 \mathrm{~Hz},{ }^{4} J_{\mathrm{H}^{\prime}-\mathrm{H} 2^{\prime}}=2.1 \mathrm{~Hz}, \mathrm{H}_{6^{\prime}}\right), 6.89\left(\mathrm{~d}, 1 \mathrm{H},{ }^{3} J_{\mathrm{H} 5^{\prime}-\mathrm{H} 6^{\prime}}=8.4 \mathrm{~Hz}, \mathrm{H}_{5^{\prime}}\right)$, $6.54\left(\mathrm{~d}, 1 \mathrm{H},{ }^{4} \mathrm{~J}_{\mathrm{H} 7-\mathrm{H} 5}=1.5 \mathrm{~Hz}, \mathrm{H}_{7}\right), 6.07$ (brs, $\left.1 \mathrm{H}, \mathrm{NH}\right), 4.76\left(\mathrm{~m}, 3 \mathrm{H}, \mathrm{H}_{\mathrm{a}}, \mathrm{H}_{\mathrm{g}}\right), 4.67\left(\mathrm{~d}, 2 \mathrm{H},{ }^{3} \mathrm{~J}_{\mathrm{Hd}-\mathrm{NH}}=\right.$ $\left.5.7 \mathrm{~Hz}, \mathrm{H}_{\mathrm{d}}\right), 4.57\left(\mathrm{~m}, 2 \mathrm{H}, \mathrm{H}_{\mathrm{f}}\right), 4.03\left(\mathrm{~m}, 2 \mathrm{H}, \mathrm{H}^{\prime}\right), 3.90\left(\mathrm{~s}, 3 \mathrm{H}, \mathrm{H}_{\mathrm{e}}\right), 1.00\left(\mathrm{~m}, 21 \mathrm{H}, \mathrm{H}_{\mathrm{b}}, \mathrm{H}_{\mathrm{c}}\right) ;{ }^{13} \mathrm{C} N M R$ $\left(50 \mathrm{MHz}, \mathrm{CDCl}_{3}\right) \delta 154.9\left(\mathrm{C}_{4^{\prime}}\right), 153.0\left(\mathrm{C}_{4}\right), 148.8\left(\mathrm{C}_{8}\right), 148.6\left(\mathrm{C}_{2}\right), 143.0\left(\mathrm{C}_{8 \mathrm{a}}\right), 132.4\left(\mathrm{C}_{1^{\prime}}\right), 129.6$ $\left(C_{2^{\prime}}\right), 127.1\left(C_{6^{\prime}}\right), 123.0\left(C_{3^{\prime}}\right), 121.1\left(C_{4 a}\right), 120.0\left(2 C, C N, C_{3}\right), 119.3\left(C_{5}\right), 112.4\left(C_{5^{\prime}}\right), 109.2\left(C_{7}\right)$, $108.0\left(C_{6}\right), 64.3\left(2 C, C_{f}, C_{f^{\prime}}\right), 63.4\left(C_{g}\right), 63.2\left(C_{a}\right), 56.4\left(C_{e}\right), 52.2\left(C_{d}\right), 18.0\left(6 C, C_{c}\right), 11.9\left(3 C, C_{b}\right)$; 
HRMS (ESI) $\mathrm{m} / \mathrm{z}$ calculated for $\mathrm{C}_{31} \mathrm{H}_{42}{ }^{35} \mathrm{CIN}_{4} \mathrm{O}_{3} \mathrm{Si}[\mathrm{M}+\mathrm{H}]^{+}$: 581.2709 , found $\mathrm{C}_{31} \mathrm{H}_{42}{ }^{35} \mathrm{ClN}_{4} \mathrm{O}_{3} \mathrm{Si}$ $[\mathrm{M}+\mathrm{H}]^{+}: 581.2711$ (100\%); $\mathrm{C}_{31} \mathrm{H}_{42}{ }^{37} \mathrm{CIN}_{4} \mathrm{O}_{3} \mathrm{Si}[\mathrm{M}+\mathrm{H}]^{+}: 583.2685$ (33\%).

\subsection{General procedure for $25 \& 26$ :}

4-Nitrobenzenesulfonyl chloride (3.0 eq.), triethylamine $(0.29 \mathrm{~mL}, 2.08 \mathrm{mmol}, 5.0$ eq.), and DMAP (0.2 eq.) were added, under stirring and argon atmosphere, to a solution of 24 (1.0 eq.) in anhydrous dichloromethane $(20 \mathrm{~mL})$. The resulting mixture was stirred at room temperature for 19 hours before addition of water $(60 \mathrm{~mL})$. This mixture was extracted with ethyl acetate $(3 \times 60 \mathrm{~mL})$. The combined organic layers were washed with brine $(100 \mathrm{~mL})$, dried over magnesium sulfate, filtered and concentrated under reduced pressure at room temperature. The crude residue was purified by column chromatography $\left(\mathrm{SiO}_{2}\right.$, ethyl acetate/cyclohexane, $2 / 8, v / v)$ to give the desired products.

\subsection{1-[4-((3-Chloro-4-methoxybenzyl)amino)-6-cyano-3-} (((triisopropylsilyl)oxy)methyl)quinolin-8-yl]azetidin-3-yl-4-methylbenzenesulfonate (25):

Yellow solid; yield $72 \%$ (0.22 g, $0.30 \mathrm{mmol}) ; \mathrm{R}_{\mathrm{f}}\left(\mathrm{SiO}_{2}\right.$, ethyl acetate / cyclohexane, 5 / 5, v / v): 0.73; Mp: $67 \pm 1{ }^{\circ} \mathrm{C}$; IR $\left(\mathrm{cm}^{-1}\right) \vee 2942\left(\mathrm{v}_{\text {as CH3 }}\right), 2864\left(\mathrm{v}_{\mathrm{s}} \mathrm{CH} 3\right), 2224\left(\mathrm{v}_{\mathrm{C}=\mathrm{N}}\right), 1502\left(\delta_{\mathrm{N}-\mathrm{H}}\right), 1437\left(\mathrm{v}_{\text {as }}\right.$ so2), 1176 ( $v_{s}$ so2), 1063 ( $\left.v_{c-o}\right), 812\left(v_{c-s i}\right) ;{ }^{1} \mathrm{H} \mathrm{NMR}\left(200 \mathrm{MHz} \mathrm{CDCl}_{3}\right) \delta 8.31\left(\mathrm{~s}, 1 \mathrm{H}, \mathrm{H}_{2}\right), 7.82(\mathrm{~m}$, $\left.3 \mathrm{H}, \mathrm{H}_{5}, \mathrm{H}_{8^{\prime}}\right), 7.38\left(\mathrm{~m}, 2 \mathrm{H}, \mathrm{H}_{9^{\prime}}\right), 7.33\left(\mathrm{~d}, 1 \mathrm{H},{ }^{4} \mathrm{JH}_{\mathrm{H}^{\prime}-\mathrm{H} 6^{\prime}}=2.2 \mathrm{~Hz}, \mathrm{H}_{2^{\prime}}\right), 7.15\left(\mathrm{dd}, 1 \mathrm{H},{ }^{3} \mathrm{JH}_{6^{\prime}-\mathrm{H} 5^{\prime}}=8.4 \mathrm{~Hz}\right.$, $\left.{ }^{4} J_{\mathrm{H}^{\prime}-\mathrm{H} 2^{\prime}}=2.2 \mathrm{~Hz}, \mathrm{H}_{6^{\prime}}\right), 6.89\left(\mathrm{~d}, 1 \mathrm{H},{ }^{3} \mathrm{JH}_{5^{\prime}-\mathrm{H}^{\prime}}=8.4 \mathrm{~Hz}, \mathrm{H}_{5^{\prime}}\right), 6.46\left(\mathrm{~s}, 1 \mathrm{H}, \mathrm{H}_{7}\right), 5.96(\mathrm{brs}, 1 \mathrm{H}, \mathrm{NH}), 5.22$ $\left(\mathrm{m}, 1 \mathrm{H}, \mathrm{H}_{\mathrm{g}}\right), 4.74\left(\mathrm{~s}, 2 \mathrm{H}, \mathrm{H}_{\mathrm{a}}\right), 4.63\left(\mathrm{~m}, 4 \mathrm{H}, \mathrm{H}_{\mathrm{d}}, \mathrm{H}_{\mathrm{f}}\right), 4.16\left(\mathrm{~m}, 2 \mathrm{H}, \mathrm{H}_{\mathrm{f}^{\prime}}\right), 3.90\left(\mathrm{~s}, 3 \mathrm{H}, \mathrm{H}_{\mathrm{e}}\right), 2.48(\mathrm{~s}, 3 \mathrm{H}$, $\left.\mathrm{H}_{\mathrm{h}}\right), 1.16\left(\mathrm{~m}, 21 \mathrm{H}, \mathrm{H}_{\mathrm{b}}, \mathrm{H}_{\mathrm{c}}\right) ;{ }^{13} \mathrm{C} \mathrm{NMR}\left(50 \mathrm{MHz}, \mathrm{CDCl}_{3}\right) \delta 154.9\left(\mathrm{C}_{4^{\prime}}\right), 152.9\left(\mathrm{C}_{4}\right), 148.6\left(\mathrm{C}_{2}\right), 147.9$ $\left(C_{8}\right), 145.5\left(C_{10^{\prime}}\right), 142.8\left(C_{8 a}\right), 133.1\left(C_{7^{\prime}}\right), 132.2\left(C_{1^{\prime}}\right), 130.2\left(2 C, C_{9^{\prime}}\right), 129.5\left(C_{2^{\prime}}\right), 128.0\left(2 C, C_{8^{\prime}}\right)$, $127.1\left(C_{6^{\prime}}\right), 123.1\left(2 \mathrm{C}, C_{3^{\prime}}, C_{4 a}\right), 120.1\left(C_{5}\right), 119.8(C N), 119.2\left(C_{3}\right), 112.4\left(C_{5^{\prime}}\right), 108.9\left(C_{7}\right), 107.9$ $\left(C_{6}\right), 69.3\left(C_{g}\right), 63.1\left(C_{a}\right), 61.2\left(2 C, C_{f}, C_{f^{\prime}}\right), 56.3\left(C_{e}\right), 52.1\left(C_{d}\right), 21.8\left(C_{h}\right), 17.9\left(6 C, C_{c}\right), 11.8(3 C$, $\left.\mathrm{C}_{\mathrm{b}}\right)$; HRMS (ESI) $\mathrm{m} / \mathrm{z}$ calculated for $\mathrm{C}_{38} \mathrm{H}_{48}{ }^{35} \mathrm{CIN}_{4} \mathrm{O}_{5} \mathrm{SSi}[\mathrm{M}+\mathrm{H}]^{+}$: 735.2798, found $\mathrm{C}_{38} \mathrm{H}_{48}{ }^{35} \mathrm{CIN}_{4} \mathrm{O}_{5} \mathrm{SSi}[\mathrm{M}+\mathrm{H}]^{+}: 735.2798$ (100\%); $\mathrm{C}_{38} \mathrm{H}_{48}{ }^{37} \mathrm{CIN}_{4} \mathrm{O}_{5} \mathrm{SSi}[\mathrm{M}+\mathrm{H}]^{+}: 737.2780$ (33\%).

\subsection{1-[4-((3-Chloro-4-methoxybenzyl)amino)-6-cyano-3-} (((triisopropylsilyl)oxy)methyl)quinolin-8-yl]azetidin-3-yl-4-nitrobenzenesulfonate (26):

Yellow solid; yield 78\% (0.30 g, $0.39 \mathrm{mmol}) ; \mathrm{R}_{\mathrm{f}}\left(\mathrm{SiO}_{2}\right.$, ethyl acetate/cyclohexane, 3/7, v/v): 0.44; Mp: $65 \pm 1{ }^{\circ} \mathrm{C}$; IR $\left(\mathrm{cm}^{-1}\right) \vee 2970\left(\mathrm{v}_{\text {as } \mathrm{CH}}\right), 2901\left(\mathrm{v}_{\mathrm{s}} \mathrm{CH} 3\right), 2232\left(\mathrm{v}_{\mathrm{C} \equiv \mathrm{N}}\right), 1259\left(\mathrm{v}_{\mathrm{C}-\mathrm{O}}\right), 1187\left(\mathrm{v}_{\mathrm{C}-\mathrm{o}}\right), 1066$ ( $\left.v_{\mathrm{c}-\mathrm{o}}\right), 810\left(\mathrm{v}_{\mathrm{c}-\mathrm{si}}\right) ;{ }^{1} \mathrm{H}$ NMR $\left(200 \mathrm{MHz}, \mathrm{CDCl}_{3}\right) \delta 8.42\left(\mathrm{~m}, 2 \mathrm{H}, \mathrm{H}_{9^{\prime}}\right), 8.29\left(\mathrm{~s}, 1 \mathrm{H}, \mathrm{H}_{2}\right), 8.14(\mathrm{~m}, 2 \mathrm{H}$, $\left.\mathrm{H}_{8^{\prime}}\right), 7.83\left(\mathrm{~d}, 1 \mathrm{H},{ }^{4} J_{\mathrm{H} 5-\mathrm{H} 7}=1.3 \mathrm{~Hz}, \mathrm{H}_{5}\right), 7.32\left(\mathrm{~d}, 1 \mathrm{H},{ }^{4} J_{\mathrm{H} 2^{\prime}-\mathrm{H} 6^{\prime}}=2.2 \mathrm{~Hz}, \mathrm{H}_{2^{\prime}}\right), 7.15\left(\mathrm{dd}, 1 \mathrm{H},{ }^{3} \mathrm{JH}_{6^{\prime}-\mathrm{H} 5^{\prime}}=\right.$ $\left.8.4 \mathrm{~Hz},{ }^{4} J_{\mathrm{H}^{\prime}-\mathrm{H} 2^{\prime}}=2.2 \mathrm{~Hz}, \mathrm{H}_{6^{\prime}}\right), 6.88\left(\mathrm{~d}, 1 \mathrm{H},{ }^{3} J_{\mathrm{H}^{\prime}-\mathrm{H}^{\prime}}=8.4 \mathrm{~Hz}, \mathrm{H}_{5^{\prime}}\right), 6.47\left(\mathrm{~d}, 1 \mathrm{H},{ }^{4} J_{\mathrm{H} 7-\mathrm{H} 5}=1.3 \mathrm{~Hz}, \mathrm{H}_{7}\right)$, 5.96 (brs, $1 \mathrm{H}, \mathrm{NH}), 5.31\left(\mathrm{~m}, 1 \mathrm{H}, \mathrm{H}_{\mathrm{g}}\right), 4.73\left(\mathrm{~s}, 2 \mathrm{H}, \mathrm{H}_{\mathrm{a}}\right), 4.65\left(\mathrm{~m}, 4 \mathrm{H}, \mathrm{H}_{\mathrm{d}}, \mathrm{H}_{\mathrm{f}}\right), 4.24\left(\mathrm{~m}, 2 \mathrm{H}, \mathrm{H}_{\mathrm{f}^{\prime}}\right), 3.88$ $\left(\mathrm{s}, 3 \mathrm{H}, \mathrm{H}_{\mathrm{e}}\right), 0.99\left(\mathrm{~m}, 21 \mathrm{H}, \mathrm{H}_{\mathrm{b}}, \mathrm{H}_{\mathrm{c}}\right) ;{ }^{13} \mathrm{CNMR}\left(50 \mathrm{MHz}, \mathrm{CDCl}_{3}\right) \delta 154.8\left(\mathrm{C}_{4^{\prime}}\right), 152.7\left(\mathrm{C}_{4}\right), 151.0\left(\mathrm{C}_{10^{\prime}}\right)$, $149.1\left(C_{2}\right), 147.6\left(C_{8}\right), 143.0\left(C_{8 a}\right), 141.9\left(C_{7^{\prime}}\right), 132.2\left(C_{1^{\prime}}\right), 129.4\left(C_{2^{\prime}}\right), 129.3\left(2 C, C_{9^{\prime}}\right), 127.0\left(C_{6^{\prime}}\right)$, $124.7\left(2 \mathrm{C}, \mathrm{C}_{8^{\prime}}\right), 123.0\left(\mathrm{C}_{3^{\prime}}\right), 121.0\left(\mathrm{C}_{4 \mathrm{a}}\right), 120.3\left(\mathrm{C}_{5}\right), 119.7(\mathrm{CN}), 119.2\left(\mathrm{C}_{3}\right), 112.4\left(\mathrm{C}_{5^{\prime}}\right), 108.8\left(\mathrm{C}_{7}\right)$, $107.7\left(C_{6}\right), 70.7\left(C_{g}\right), 63.1\left(C_{a}\right), 61.1\left(2 C, C_{f}, C_{f}\right), 56.3\left(C_{e}\right), 52.0\left(C_{d}\right), 17.9\left(6 C, C_{c}\right), 11.8\left(3 C, C_{b}\right)$; HRMS (ESI) $\mathrm{m} / \mathrm{z}$ calculated for $\mathrm{C}_{37} \mathrm{H}_{45}{ }^{35} \mathrm{CIN}_{5} \mathrm{O}_{7} \mathrm{SSi}[\mathrm{M}+\mathrm{H}]^{+}: 766.2492$, found $\mathrm{C}_{37} \mathrm{H}_{45}{ }^{35} \mathrm{ClN}_{5} \mathrm{O}_{7} \mathrm{SSi}$ $[\mathrm{M}+\mathrm{H}]^{+}: 766.2492$ (100\%); $\mathrm{C}_{37} \mathrm{H}_{45}{ }^{37} \mathrm{CIN}_{5} \mathrm{O}_{7} \mathrm{SSi}[\mathrm{M}+\mathrm{H}]^{+}: 768.2475$ (33\%). 


\subsection{General procedure for $27 \& 28$ :}

A 1.0 M solution of tetra- $n$-butylammonium fluoride in tetrahydrofuran $(0.39 \mathrm{~mL}, 0.39 \mathrm{mmol}$, 1.0 eq.) was added slowly at $0{ }^{\circ} \mathrm{C}$, under stirring and argon atmosphere, to a solution of $\mathbf{2 5}$ or 26 (1.0 eq.) in anhydrous THF $(20 \mathrm{~mL})$. The resulting mixture was stirred at room temperature for 30 minutes before addition of water at $0{ }^{\circ} \mathrm{C}$. The resulting solution was extracted with ethyl acetate $(3 \times 50 \mathrm{~mL})$ and the combined organic layers were washed with brine $(100 \mathrm{~mL})$, dried over magnesium sulfate, filtered and concentrated under reduced pressure at room temperature. The crude residue was purified by column chromatography $\left(\mathrm{SiO}_{2}\right.$, ethyl acetate/cyclohexane, 5/5, v/v) to give the desired product.

\subsection{1-[4-((3-Chloro-4-methoxybenzyl)amino)-6-cyano-3-(hydroxymethyl)quinolin- 8-yl]azetidin-3-yl-4-methylbenzenesulfonate (27):}

Light yellow solid; yield 70\% (0.12 g, $0.21 \mathrm{mmol}) ; \mathrm{R}_{\mathrm{f}}\left(\mathrm{SiO}_{2}\right.$, methanol/dichloromethane, 3/7, v/v): 0.39; Mp: $155 \pm 1{ }^{\circ} \mathrm{C}$; IR $\left(\mathrm{cm}^{-1}\right)$ v $2226\left(\mathrm{v}_{\mathrm{C} \equiv \mathrm{N}}\right), 1502\left(\delta_{\mathrm{N}-\mathrm{H}}\right), 1349$ ( $\left.\mathrm{v}_{\text {as SO2 }}\right), 1173\left(\mathrm{v}_{\mathrm{s}} \mathrm{sO2}\right), 1064$ (vc-o), $812\left(\mathrm{v}_{\mathrm{c}-\mathrm{cl}}\right) ;{ }^{1} \mathrm{H}$ NMR $\left(200 \mathrm{MHz}, \mathrm{CDCl}_{3}\right) \delta 8.33\left(\mathrm{~s}, 1 \mathrm{H}, \mathrm{H}_{2}\right), 7.83\left(\mathrm{~d}, 2 \mathrm{H},{ }^{3} \mathrm{JH}_{\mathrm{H}^{\prime}-\mathrm{H9^{ \prime }}}=8.4 \mathrm{~Hz}, \mathrm{H}_{8^{\prime}}\right)$, $7.74\left(\mathrm{~d}, 1 \mathrm{H},{ }^{4} \mathrm{~J}_{\mathrm{H} 5-\mathrm{H} 7}=1.3 \mathrm{~Hz}, \mathrm{H}_{5}\right), 7.39\left(\mathrm{~d}, 2 \mathrm{H},{ }^{3} \mathrm{~J}_{\mathrm{H}^{\prime}-\mathrm{H} 8^{\prime}}=8.4 \mathrm{~Hz}, \mathrm{H}_{9^{\prime}}\right), 7.32\left(\mathrm{~d}, 1 \mathrm{H},{ }^{4} \mathrm{~J}_{\mathrm{Hz}}-\mathrm{H}^{\prime}=2.2 \mathrm{~Hz}\right.$, $\left.\mathrm{H}_{2^{\prime}}\right), 7.16\left(\mathrm{dd}, 1 \mathrm{H},{ }^{3} J_{\mathrm{H} 6^{\prime}-\mathrm{H} 5^{\prime}}=8.4 \mathrm{~Hz},{ }^{4} J_{\mathrm{H}^{\prime}-\mathrm{H} 2^{\prime}}=2.2 \mathrm{~Hz}, \mathrm{H}_{6^{\prime}}\right), 6.91\left(\mathrm{~d}, 1 \mathrm{H},{ }^{3} \mathrm{JH}^{\prime}-\mathrm{H}^{\prime}=8.4 \mathrm{~Hz}, \mathrm{H}_{5^{\prime}}\right), 6.48$ $\left(\mathrm{d}, 1 \mathrm{H},{ }^{4} J_{\mathrm{H} 7 \mathrm{H} 5}=1.3 \mathrm{~Hz}, \mathrm{H}_{7}\right), 5.84$ (brs, $\left.1 \mathrm{H}, \mathrm{NH}\right), 5.22\left(\mathrm{~m}, 1 \mathrm{H}, \mathrm{H}_{\mathrm{g}}\right), 4.68\left(\mathrm{~s}, 4 \mathrm{H}, \mathrm{H}_{\mathrm{a}}, \mathrm{H}_{\mathrm{b}}\right), 4.59(\mathrm{~m}$, $\left.2 \mathrm{H}, \mathrm{H}_{\mathrm{d}}\right), 4.14\left(\mathrm{~m}, 2 \mathrm{H}, \mathrm{H}_{\mathrm{d}^{\prime}}\right), 3.90\left(\mathrm{~s}, 3 \mathrm{H}, \mathrm{H}_{\mathrm{c}}\right), 2.49\left(\mathrm{~s}, 3 \mathrm{H}, \mathrm{H}_{\mathrm{h}}\right) ;{ }^{13} \mathrm{C} \mathrm{NMR}\left(50 \mathrm{MHz}, \mathrm{CDCl}_{3}\right) \delta 154.8$ $\left(C_{4^{\prime}}\right), 152.4\left(C_{4}\right), 149.8\left(C_{2}\right), 147.8\left(C_{8}\right), 145.6\left(C_{10^{\prime}}\right), 142.5\left(C_{8 a}\right), 133.1\left(C_{7^{\prime}}\right), 132.1\left(C_{1^{\prime}}\right), 130.2$ $\left(2 \mathrm{C}, \mathrm{C}_{9^{\prime}}\right), 129.4\left(\mathrm{C}_{2^{\prime}}\right), 128.1\left(2 \mathrm{C}, \mathrm{C}_{8^{\prime}}\right), 126.9\left(\mathrm{C}_{6^{\prime}}\right), 122.9\left(\mathrm{C}_{3^{\prime}}\right), 120.9\left(\mathrm{C}_{4 \mathrm{a}}\right), 119.7(\mathrm{CN}), 119.3\left(\mathrm{C}_{5}\right)$, $118.5\left(C_{3}\right), 112.5\left(C_{5^{\prime}}\right), 109.2\left(C_{7}\right), 108.1\left(C_{6}\right), 69.3\left(C_{e}\right), 61.9\left(C_{a}\right), 61.3\left(2 C, C_{d}, C_{d^{\prime}}\right), 56.3\left(C_{c}\right)$, $51.8\left(\mathrm{C}_{\mathrm{b}}\right), 21.9\left(\mathrm{C}_{\mathrm{f}}\right)$; HRMS (ESI) $\mathrm{m} / \mathrm{z}$ calculated for $\mathrm{C}_{29} \mathrm{H}_{28}{ }^{35} \mathrm{CIN}_{4} \mathrm{O}_{5} \mathrm{~S}[\mathrm{M}+\mathrm{H}]^{+}: 579.1463$, found $\mathrm{C}_{29} \mathrm{H}_{28}{ }^{35} \mathrm{CIN}_{4} \mathrm{O}_{5} \mathrm{~S}[\mathrm{M}+\mathrm{H}]^{+}: 579.1473$ (100\%); $\mathrm{C}_{29} \mathrm{H}_{28}{ }^{37} \mathrm{CIN}_{4} \mathrm{O}_{5} \mathrm{~S}[\mathrm{M}+\mathrm{H}]^{+}: 581.1436$ (33\%).

\subsection{1-[4-((3-Chloro-4-methoxybenzyl)amino)-6-cyano-3-(hydroxymethyl)quinolin- 8-yl]azetidin-3-yl-4-nitrobenzenesulfonate (28):}

Light yellow solid; yield 64\% (0.15 g, $0.25 \mathrm{mmol}) ; \mathrm{R}_{\mathrm{f}}\left(\mathrm{SiO}_{2}\right.$, methanol/dichloromethane, 5/5, v/v): 0.21; Mp: $128 \pm 1{ }^{\circ} \mathrm{C} ; \mathrm{IR}\left(\mathrm{cm}^{-1}\right)$ v $2226\left(\mathrm{v}_{\mathrm{C} \equiv \mathrm{N}}\right), 1502\left(\delta_{\mathrm{NH}}\right), 1347$ ( $\left.\mathrm{vas} \mathrm{SO}_{2}\right), 1187\left(\mathrm{v}_{\mathrm{s}} \mathrm{sO}\right), 1063$ (vc-o), $1014\left(\mathrm{vc}_{\mathrm{c}-\mathrm{o}}\right) ;{ }^{1} \mathrm{H}$ NMR $\left(200 \mathrm{MHz}, \mathrm{CDCl}_{3}\right) \delta 8.42\left(\mathrm{~d}, 2 \mathrm{H},{ }^{3} \mathrm{JH}^{\prime}-\mathrm{H}^{\prime}=8.4 \mathrm{~Hz}, \mathrm{H}_{9^{\prime}}\right), 8.28(\mathrm{~s}, 1 \mathrm{H}$, $\left.\mathrm{H}_{2}\right), 8.14\left(\mathrm{~d}, 1 \mathrm{H},{ }^{3} \mathrm{~J}_{\mathrm{H} 8^{\prime}-\mathrm{H} g^{\prime}}=8.4 \mathrm{~Hz}, \mathrm{H}_{8^{\prime}}\right), 7.72\left(\mathrm{~s}, 1 \mathrm{H}, \mathrm{H}_{5}\right), 7.30\left(\mathrm{~d}, 1 \mathrm{H},{ }^{4} \mathrm{JH}_{\mathrm{H}^{\prime}-\mathrm{H} 6^{\prime}}=2.1 \mathrm{~Hz}, \mathrm{H}_{2^{\prime}}\right), 7.15$ $\left(\mathrm{dd}, 1 \mathrm{H},{ }^{3} J_{\mathrm{H} 6^{\prime}-\mathrm{H} 5^{\prime}}=8.5 \mathrm{~Hz},{ }^{4} J_{\mathrm{H} 6^{\prime}-\mathrm{H} 2^{\prime}}=2.1 \mathrm{~Hz}, \mathrm{H}_{6^{\prime}}\right), 6.89\left(\mathrm{~d}, 1 \mathrm{H},{ }^{3} \mathrm{~J}_{\mathrm{H} 5^{\prime}-\mathrm{H} 6^{\prime}}=8.5 \mathrm{~Hz}, \mathrm{H}_{5^{\prime}}\right), 6.46\left(\mathrm{~s}, 1 \mathrm{H}, \mathrm{H}_{7}\right)$, $5.81(\mathrm{se}, 1 \mathrm{H}, \mathrm{NH}), 5.30\left(\mathrm{~m}, 1 \mathrm{H}, \mathrm{H}_{\mathrm{g}}\right), 4.66\left(\mathrm{~m}, 4 \mathrm{H}, \mathrm{H}_{\mathrm{a}}, \mathrm{H}_{\mathrm{b}}\right), 4.59\left(\mathrm{~m}, 2 \mathrm{H}, \mathrm{H}_{\mathrm{d}}\right), 4.20\left(\mathrm{~m}, 2 \mathrm{H}, \mathrm{H}_{\mathrm{d}^{\prime}}\right), 3.89$ $\left(\mathrm{s}, 3 \mathrm{H}, \mathrm{H}_{\mathrm{c}}\right) ;{ }^{13} \mathrm{CNMR}\left(50 \mathrm{MHz}, \mathrm{CDCl}_{3}\right) \delta 154.9\left(\mathrm{C}_{4^{\prime}}\right), 152.5\left(\mathrm{C}_{4}\right), 151.1\left(\mathrm{C}_{10^{\prime}}\right), 149.9\left(\mathrm{C}_{2}\right), 147.4\left(\mathrm{C}_{8}\right)$, $142.3\left(C_{8 \mathrm{a}}\right), 141.8\left(\mathrm{C}_{7^{\prime}}\right), 132.0\left(\mathrm{C}_{1^{\prime}}\right), 129.4\left(2 \mathrm{C}, \mathrm{C}_{8^{\prime}}\right), 129.3\left(\mathrm{C}_{2^{\prime}}\right), 126.9\left(\mathrm{C}_{6^{\prime}}\right), 124.8\left(2 \mathrm{C}, \mathrm{C}_{9^{\prime}}\right), 123.0$ $\left(C_{3^{\prime}}\right), 120.8\left(C_{4 a}\right), 119.7\left(C_{5}\right), 119.6(C N), 118.4\left(C_{3}\right), 112.5\left(C_{5^{\prime}}\right), 109.5\left(C_{7}\right), 108.1\left(C_{6}\right), 70.7\left(C_{e}\right)$, $61.9\left(\mathrm{C}_{\mathrm{a}}\right), 61.2\left(2 \mathrm{C}, \mathrm{C}_{d}, \mathrm{C}_{\mathrm{d}^{\prime}}\right), 56.4\left(\mathrm{C}_{\mathrm{c}}\right), 51.8\left(\mathrm{C}_{\mathrm{b}}\right)$; HRMS (ESI) $\mathrm{m} / z$ calculated for $\mathrm{C}_{28} \mathrm{H}_{25}{ }^{35} \mathrm{CIN}_{5} \mathrm{O}_{7} \mathrm{~S}$ $[\mathrm{M}+\mathrm{H}]^{+}: 610.1158$, found $\mathrm{C}_{28} \mathrm{H}_{25}{ }^{35} \mathrm{ClN}_{5} \mathrm{O}_{7} \mathrm{~S}[\mathrm{M}+\mathrm{H}]^{+}: 610.1161(100 \%) ; \mathrm{C}_{28} \mathrm{H}_{25}{ }^{37} \mathrm{CIN}_{5} \mathrm{O}_{7} \mathrm{~S}[\mathrm{M}+\mathrm{H}]^{+}$: $612.1132(33 \%)$.

\subsection{Radiochemistry}




\subsubsection{General}

No-carrier-added $\left[{ }^{18} \mathrm{~F}\right]$ fluoride was produced via the $\left[{ }^{18} \mathrm{O}(\mathrm{p}, \mathrm{n}){ }^{18} \mathrm{~F}\right]$ nuclear reaction by irradiation of an $\left[{ }^{18} \mathrm{O}\right] \mathrm{H}_{2} \mathrm{O}$ target (Hyox 18 enriched water, Rotem Industries Ltd, Israel) on a Cyclone 18/9 (iba RadioPharma Solutions, Belgium) with fixed energy proton beam using Nirta $\left[{ }^{18} \mathrm{~F}\right]$ fluoride XL target.

Radio thin layer chromatography (radio-TLC) was performed on silica gel (Polygram $^{\circledR} \mathrm{SIL}$ $\mathrm{G} / \mathrm{UV}_{254}$ ) pre-coated plates with a mixture of ethyl acetate/ $n$-hexane $4 / 1(\mathrm{v} / \mathrm{v})$ as eluent. The plates were exposed to storage phosphor screens (BAS-IP MS 2025, FUJIFILM Co., Tokyo, Japan) and recorded using the Amersham Typhoon RGB Biomolecular Imager (GE Healthcare Life Sciences). Images were quantified with the ImageQuant TL8.1 software (GE Healthcare Life Sciences).

Analytical chromatographic separations were performed on a JASCO LC-2000 system, incorporating a PU-2080Plus pump, AS-2055Plus auto injector (100 $\mu \mathrm{L}$ sample loop), and a UV2070Plus detector coupled with a gamma radioactivity HPLC detector (Gabi Star, raytest Isotopenmessgeräte $\mathrm{GmbH}$ ). Data analysis was performed with the Galaxie chromatography software (Agilent Technologies) using the chromatograms obtained at $254 \mathrm{~nm}$. A Reprosil-Pur C18-AQ column ( $250 \times 4.6 \mathrm{~mm}$; $5 \mu \mathrm{m}$; Dr. Maisch HPLC GmbH; Germany) with ACN/20 mM $\mathrm{NH}_{4} \mathrm{OAc}$ aq. ( $\mathrm{pH} \mathrm{6.8)}$ ) as eluent mixture and a flow of $1.0 \mathrm{~mL} / \mathrm{min}$ was used (gradient: eluent $\mathrm{A}$ $10 \% \mathrm{ACN} / 20 \mathrm{mM} \mathrm{NH}_{4} \mathrm{OAc}$ aq.; eluent B 90\% ACN/20 mM NH $\mathrm{HOAc}_{4}$ aq.; 0-10 min 100\% A, 10$30 \mathrm{~min}$ up to $100 \% \mathrm{~B}, 30-35 \mathrm{~min} 100 \% \mathrm{~B}, 35-37 \mathrm{~min}$ up to $100 \% \mathrm{~A}, 37-45 \min 100 \% \mathrm{~A}$ ).

The ammonium acetate and sodium dodecyl sulfate (SDS) concentrations stated as $20 \mathrm{mM}$ $\mathrm{NH}_{4} \mathrm{OAc}$ aq. and $100 \mathrm{mM}$ aq., respectively, correspond to the concentration in the aqueous component of an eluent mixture.

The molar activities were determined on the basis of a calibration curve carried out under isocratic HPLC conditions ( $50 \% \mathrm{ACN} / 20 \mathrm{mM} \mathrm{NH}_{4} \mathrm{OAC}_{\mathrm{aq}}$; Reprosil-Pur C18-AQ, $250 \times 4.6 \mathrm{~mm}$ ) using chromatograms obtained at $254 \mathrm{~nm}$.

\subsubsection{Radiosyntheses}

\subsubsection{Manual syntheses with tosylate 27 as precursor}

No carrier added $\left[{ }^{18} \mathrm{~F}\right]$ fluoride in $1.5 \mathrm{~mL}$ of water was trapped on a Chromafix ${ }^{\circledR} 30 \mathrm{PS}^{-\mathrm{HCO}_{3}}{ }^{-}$ cartridge (ABX, Radeberg, Germany). The activity was eluted with $300 \mu \mathrm{L}$ of an aqueous solution of potassium carbonate $\left(\mathrm{K}_{2} \mathrm{CO}_{3}, 1.8 \mathrm{mg}, 13 \mu \mathrm{mol}\right)$ into a $4 \mathrm{~mL} \mathrm{~V}$-vial and Kryptofix 2.2 .2 $\left(\mathrm{K}_{2.2 .2}, 11 \mathrm{mg}, 29 \mu \mathrm{mol}\right)$ in $1 \mathrm{~mL}$ of $\mathrm{ACN}$ was added. The aqueous $\left[{ }^{18} \mathrm{~F}\right]$ fluoride was azeotropically dried under vacuum and nitrogen flow within 7-10 min using a single mode microwave (75 W, at $50-60{ }^{\circ} \mathrm{C}$, power cycling mode; Discover PETWave from CEM corporation, USA). Two aliquots of $A C N(2 \times 1.0 \mathrm{~mL})$ were added during the drying procedure and the final complex was dissolved in $1.0 \mathrm{~mL}$ of labeling solvent and divided in two portions. Accordingly, most of the labeling reactions were performed with half of the $\mathrm{K}\left[{ }^{18} \mathrm{~F}\right] \mathrm{F} / \mathrm{K}_{222}$ complex. Thereafter, $\mathrm{a}$ solution of $2.0-2.5 \mathrm{mg}$ of precursor 27 in $500 \mu \mathrm{L}$ of the appropriate solvent was added, and ${ }^{18} \mathrm{~F}$-labeling was performed at different temperatures in dependence of the solvent used $(90$, $100,120,130$ and $150{ }^{\circ} \mathrm{C}$ ). To analyze the reaction mixture and to determine radiochemical 
yields, samples were taken for radio-HPLC and radio-TLC at different time points $(5,10,15,20$ and $25 \mathrm{~min}$ ).

\subsubsection{Automated syntheses with tosylate 27 as precursor}

Remote controlled radiosynthesis of $\left[{ }^{18} \mathrm{~F}\right] 7$ was performed using a TRACERlab FX2 N synthesizer (GE Healthcare, USA) equipped with a Laboport vacuum pump N810.3FT.18 (KNF Neuburger GmbH, Freiburg, Germany), a BlueShadow UV detector 10D (KNAUER GmbH, Berlin, Germany) and the TRACERlab FX Software.

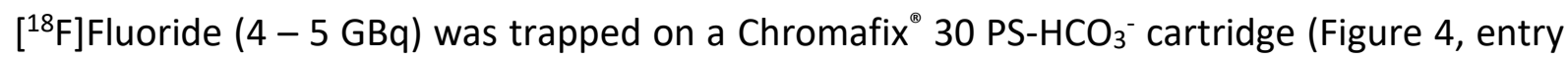
1, ABX, Radeberg, Germany) and eluted into the reactor with $400 \mu \mathrm{L}$ of an aqueous solution of potassium carbonate $\left(\mathrm{K}_{2} \mathrm{CO}_{3}, 1.8 \mathrm{mg}, 13 \mu \mathrm{mol}\right.$, entry 2). After adding Kryptofix 2.2.2. in $1 \mathrm{~mL}$ of ACN (11 mg, $29 \mu \mathrm{mol}$, entry 3 ), the mixture was azeotropically dried for approximately 10 minutes. Thereafter, $2.0-2.5 \mathrm{mg}$ of the tosylate precursor 27 dissolved in $1 \mathrm{~mL}$ of DMSO (entry 4) was added, and the reaction mixture was stirred at $100{ }^{\circ} \mathrm{C}$ for $15 \mathrm{~min}$. After cooling, the reaction mixture was diluted with $2.0 \mathrm{~mL}$ of $\mathrm{H}_{2} \mathrm{O}$ and $1.0 \mathrm{~mL}$ of $\mathrm{ACN}$ (entry 5 ) and transferred into the injection vial (entry 6). Semi-preparative HPLC was performed using a Reprosil-Pur C18-AQ column ( $250 \times 10 \mathrm{~mm}$; $10 \mu \mathrm{m}$; Dr. Maisch HPLC GmbH, Germany) with a solvent composition of $55 \% \mathrm{ACN} / 40 \mathrm{mM} \mathrm{NH}_{4} \mathrm{OAC}$ aq. at a flow rate of $3.5 \mathrm{~mL} / \mathrm{min}$ (entry 7). $\left[{ }^{18} \mathrm{~F}\right] 7$ was collected in the dilution vessel (entry 8) previously loaded with $40 \mathrm{~mL}$ of $\mathrm{H}_{2} \mathrm{O}$. Final purification was performed by passing the solution through a Sep-Pak ${ }^{\circledR}$ C18 light cartridge (entry 9), followed by washing with $2 \mathrm{~mL}$ of water (entry 10 ) and elution of $\left[{ }^{18} \mathrm{~F}\right] 7$ with $1.2 \mathrm{~mL}$ of EtOH (entry 11) into the product vial (entry 12). The ethanolic solution was transferred in a neighboring hotcell by remote control and the solvent manually reduced under a gentle argon stream at $75^{\circ} \mathrm{C}$ to a final volume of $10-50 \mu \mathrm{L}$. Afterwards the radiotracer was diluted in isotonic saline to obtain a final product containing $10 \%$ of EtOH (v/v).

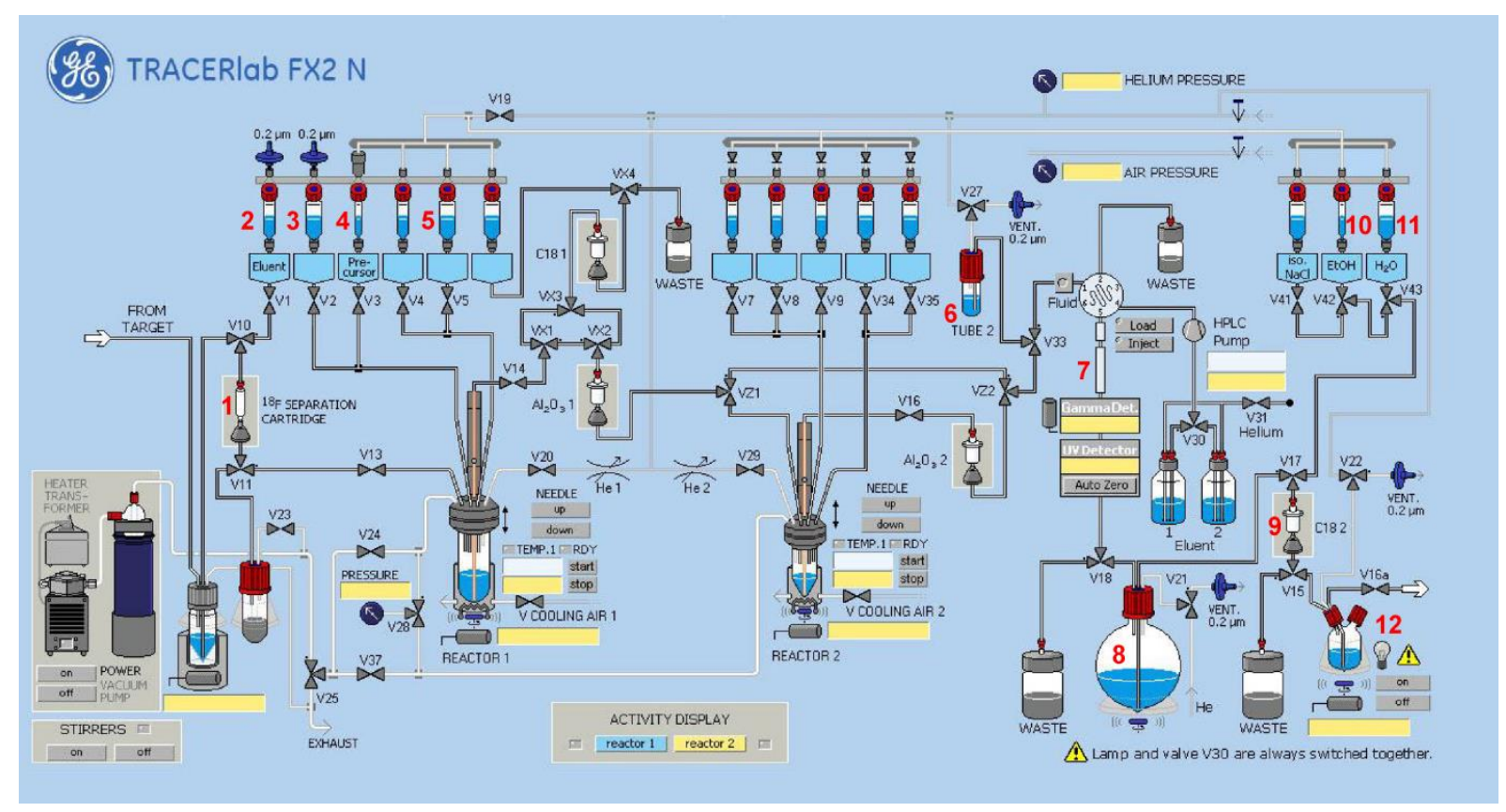


Figure 4. Scheme of the synthesis module TRACERlab FX2 $\mathrm{N}$ for the radiosynthesis of $\left[{ }^{18} \mathrm{~F}\right] 7$.

Tosylate 27 as precursor: (1) Chromafix 30-PS- $\mathrm{HCO}_{3}{ }^{-}$, (2) $\mathrm{K}_{2} \mathrm{CO}_{3}$ (1.8 mg in $400 \mu \mathrm{L}$ water), (3) $\mathrm{K}_{2.2 .2 .}$ (11 mg in $1 \mathrm{~mL}$ ACN), (4) precursor (2 mg of 27 in $1 \mathrm{~mL}$ DMSO), (5) $2.0 \mathrm{~mL}$ water and $1.0 \mathrm{~mL} \mathrm{ACN},(6)$ injection vial, (7) ReprosilPur C18-AQ (55\% ACN/40 mM NH $4{ }_{4} \mathrm{OACaq}$, flow $3.5 \mathrm{~mL} / \mathrm{min}$ ), (8) $40 \mathrm{~mL}$ water, (9) Sep-Pak ${ }^{\circledR}$ C18 light, (10) $2 \mathrm{~mL}$ water, (11) $1.2 \mathrm{~mL}$ EtOH, (12) product vial.

Nosylate 28 as precursor: (1) Sep-Pak ${ }^{\circledR}$ Accell QMA light cartridge, (2) $150 \mu \mathrm{L} \mathrm{TBAHCO}_{3}$ in $300 \mu$ water and 650 $\mu \mathrm{ACN},(3) 2 \mathrm{~mL} \mathrm{ACN}$, (4) precursor (2 mg of 28 in $200 \mu \mathrm{L} \mathrm{ACN}+700 \mu \mathrm{L} 3 \mathrm{M} 3 \mathrm{P}+80 \mu \mathrm{L}$ water), (5) $2.0 \mathrm{~mL}$ water + $2.0 \mathrm{~mL} \mathrm{ACN}$, (6) injection vial, (7) Reprosil-Pur C18-AQ (55\% ACN/40 mM NH $4 \mathrm{OACaq.,} \mathrm{flow} 2.8 \mathrm{~mL} / \mathrm{min}$ ), (8) $40 \mathrm{~mL}$ water, (9) Sep-Pak ${ }^{\circledR}$ C18 light, (10) $2 \mathrm{~mL}$ water, (11) $1.2 \mathrm{~mL}$ EtOH, (12) product vial.

\subsubsection{Manual syntheses with nosylate 28 as precursor}

No carrier added $\left[{ }^{18} \mathrm{~F}\right]$ fluoride in $1.5 \mathrm{~mL}$ of water was trapped on a Chromafix ${ }^{\circledR} 30 \mathrm{PS}^{-\mathrm{HCO}_{3}}{ }^{-}$ cartridge ( $A B X \mathrm{GmbH}$, Radeberg, Germany). The activity was eluted with $300 \mu \mathrm{L}$ of an aqueous solution of potassium carbonate $\left(\mathrm{K}_{2} \mathrm{CO}_{3}, 1.8 \mathrm{mg}, 13 \mu \mathrm{mol}\right)$ into a $4 \mathrm{~mL} \mathrm{~V}$-vial and Kryptofix 2.2 .2 $\left(\mathrm{K}_{2.2 .2}, 11 \mathrm{mg}, 29 \mu \mathrm{mol}\right)$ in $1 \mathrm{~mL}$ of $\mathrm{ACN}$ was added. For investigations of the precursor to base ratio, the amount of potassium carbonate was accordingly reduced or increased. For labeling with $\left[{ }^{18} \mathrm{~F}\right] \mathrm{TBAF}$, the tetra-n-butylammonium hydrogene carbonate $\left(\mathrm{TBAHCO}_{3}\right)$ solution $(0.075$ $M, A B X$, Radeberg, Germany) was directly placed into the $V$-vial containing $\left[{ }^{18} \mathrm{~F}\right]$ fluoride and 1 $\mathrm{mL}$ of $\mathrm{ACN}$. The aqueous $\left[{ }^{18} \mathrm{~F}\right]$ fluoride was then azeotropically dried under vacuum and nitrogen flow within 7-10 min using a single mode microwave $\left(75 \mathrm{~W}\right.$, at $50-60{ }^{\circ} \mathrm{C}$, power cycling mode). Two aliquots of $\mathrm{ACN}(2 \times 1.0 \mathrm{~mL})$ were added during the drying procedure and the final complex was dissolved in an appropriate volume of labeling solvent and used directly or divided in several portions. Thereafter, a solution of $2.0-2.5 \mathrm{mg}$ of precursor 28 in 300 $500 \mu \mathrm{L}$ of an appropriate solvent was added, and ${ }^{18} \mathrm{~F}$-labeling was performed at different temperatures in dependence of the solvent used $\left(90,100,120,130\right.$ and $\left.150{ }^{\circ} \mathrm{C}\right)$. To analyze the reaction mixture and to determine radiochemical yields, samples were taken for radioHPLC and radio-TLC at different time points $(5,10,15,20$ and $25 \mathrm{~min})$.

\subsubsection{Automated syntheses with nosylate 28 as precursor}

Remote controlled radiosynthesis of $\left[{ }^{18} \mathrm{~F}\right] 7$ was performed using a TRACERlab FX2 N synthesizer (GE Healthcare, USA) equipped with a Laboport vacuum pump N810.3FT.18 (KNF Neuburger GmbH, Freiburg, Germany), a BlueShadow UV detector 10D (KNAUER GmbH, Berlin, Germany) and the TRACERlab FX Software.

$\left[{ }^{18} \mathrm{~F}\right.$ Fluoride $\left(4-5 \mathrm{GBq}\right.$ ) was trapped on a Sep-Pak ${ }^{\circledR}$ Accell QMA light cartridge (Figure 5, entry 1, Waters, USA) and eluted into the reactor with a solution of $150 \mu \mathrm{L}$ of $\mathrm{TBAHCO}_{3}$ (entry 2, $0.075 \mathrm{M}, \mathrm{ABX} \mathrm{GmbH}$, Radeberg, Germany), $300 \mu \mathrm{L}$ of water and $650 \mu \mathrm{L}$ of ACN. After azeotropic drying at $50^{\circ} \mathrm{C}$ for $3 \mathrm{~min}, 2 \mathrm{~mL} \mathrm{ACN}$ (entry 3) were added and azeotropic drying was continued for further $3 \mathrm{~min}$ at $70^{\circ} \mathrm{C}$ and $1 \mathrm{~min}$ at $40^{\circ} \mathrm{C}$. Thereafter, $2.0-2.5 \mathrm{mg}$ of the nosylate precursor (28) dissolved in a mixture of $200 \mu \mathrm{L} \mathrm{ACN} \mathrm{/} 700 \mu \mathrm{L} 3 \mathrm{M} 3 \mathrm{P} / 80 \mu \mathrm{L}$ water (entry 4) was added, and the reaction mixture was stirred at $125^{\circ} \mathrm{C}$ for $10 \mathrm{~min}$. After cooling to $40{ }^{\circ} \mathrm{C}$, the solvent was evaporated under vacuum and helium stream for $7 \mathrm{~min}$, the residue was diluted with $2.0 \mathrm{~mL}$ of $\mathrm{H}_{2} \mathrm{O}$ and $2.0 \mathrm{~mL}$ of $\mathrm{ACN}$ (entry 5) and transferred into the injection vial (entry 6). Semi-preparative HPLC was performed using a Reprosil-Pur C18-AQ column (250 x 10 mm; 
$10 \mu \mathrm{m}$; Dr. Maisch HPLC GmbH; Germany) with a solvent composition of 55\% ACN/40 mM $\mathrm{NH}_{4} \mathrm{OAC}$ aq. at a flow rate of $2.8 \mathrm{~mL} / \mathrm{min}$ (entry 7). [ $\left.{ }^{18} \mathrm{~F}\right] 7$ was collected in the dilution vessel (entry 8) previously loaded with $40 \mathrm{~mL}$ of $\mathrm{H}_{2} \mathrm{O}$. Final purification was performed by passing the solution through a Sep-Pak ${ }^{\circledR} \mathrm{C} 18$ light cartridge (entry 9), followed by washing with $2 \mathrm{~mL}$ of water (entry 10) and elution of $\left[{ }^{18} \mathrm{~F}\right] 7$ with $1.2 \mathrm{~mL}$ of EtOH (entry 11) into the product vial (entry 12). The ethanolic solution was transferred in a neighboring hotcell by remote control and the solvent manually reduced under a gentle argon stream at $75{ }^{\circ} \mathrm{C}$ to a final volume of 10-50 $\mu \mathrm{L}$. Afterwards the radiotracer was diluted in isotonic saline to obtain a final product containing $10 \%$ of EtOH (v/v).

\subsubsection{In vitro stability}

The in vitro stability of $\left[{ }^{18} \mathrm{~F}\right] 7$ was investigated by incubation of small tracer amounts ( $\left.5 \mathrm{MBq}\right)$ at $40{ }^{\circ} \mathrm{C}$ in $500 \mu \mathrm{L}$ of phosphate buffered saline and $500 \mu \mathrm{L}$ of pig plasma samples. After 30 and 60 minutes, aliquots were taken and analyzed by radio-TLC and radio-HPLC.

\subsection{In vitro autoradiographic studies of $\left[{ }^{18} \mathrm{~F}\right] 7$}

Brain sections $(20 \mu \mathrm{m})$ of flash-frozen brains of female domestic pigs (Sus s. domestica, 6 weeks, 12-14 kg) were cut using a cryostat, thaw-mounted onto microscope slides, and after air-drying stored at $-80^{\circ} \mathrm{C}$ until use. Briefly, the brain sections were allowed to thaw in air, and pre-incubated once in $50 \mathrm{mM}$ TRIS buffer $(\mathrm{pH}$ 7.4) to remove endogenous ligand. The sections were then incubated with $2.02 \pm 0.75 \mathrm{nM}$ of $\left[{ }^{18} \mathrm{~F}\right] 7$ in TRIS buffer (50 mM TRIS- $\mathrm{HCl}$, $120 \mathrm{mM} \mathrm{NaCl}, 5 \mathrm{mM} \mathrm{KCl}, 2 \mathrm{mM} \mathrm{MgCl} 2,2 \mathrm{mM} \mathrm{CaCl} 2,0.1 \% \mathrm{BSA} \mathrm{pH} \mathrm{7.4)} \mathrm{for} 60$ minutes at room temperature. Nonspecific binding was determined in the presence of $1 \mu \mathrm{M} 7$. Displacement of $\left[{ }^{18} \mathrm{~F}\right] 7$ was also evaluated with $1 \mu \mathrm{M}$ sildenafil. Subsequently, the sections were washed twice for 2 minutes in ice-cold TRIS buffer, and dipped for 5 seconds in ice-cold distilled water. The sections were rapidly dried in a stream of cold air before being exposed to an imaging plate. Developed autoradiographs were analysed in a phosphor imager (Fuji BAS 1800 II). The quantification was performed by using 2D-densitometric analysis (AIDA 2.31 software; raytest Isotopenmessgeräte $\mathrm{GmbH}$, Germany).

\subsection{In vivo studies of $\left[{ }^{18} \mathrm{~F}\right] 7$ in mice}

All animal studies followed the international guidelines of animal care and the study protocols were approved by the Landesdirektion Leipzig, the local authority for animal care (Reg.-Nr.: TVV 08/13; Reference number: 24-9168.11/18/8). All animal experiments were performed with female CD-1 mice, 10-12 weeks old, obtained from the Experimental Centre of the Faculty of Medicine (MEZ) at the Leipzig University, Leipzig, Germany.

\subsubsection{Organ uptake}

$\left[{ }^{18} \mathrm{~F}\right] 7$ obtained as described in 4.2.2.4. was dissolved in $200 \mu \mathrm{L}$ of sterile isotonic saline and administered as a bolus injection via the tail vein of the restrained animal. At $5(n=1$; dose: $0.61 \mathrm{MBq}$ ) and 30 minutes p.i. ( $\mathrm{n}=2$; dose: 38 and $49 \mathrm{MBq}$ ), retro-orbital blood samples were obtained from the anesthetized animals. Immediately afterwards, organs of interest were 
isolated and blood plasma was obtained by centrifugation of the whole blood sample (15000 rpm, room temperature, 1 minute). All samples were weighed and the radioactivity (in CPM) was measured in a gamma-counter (Wizard 2480, PerkinElmer LAS GmbH, Germany). For conversion of CPM values to $\mathrm{Bq}$, standard samples were measured in a dose calibrator (ISOMED 2010, MED Nuklear-Medizintechnik Dresden GmbH, Dresden, Germany) as well as by gamma-counting. For each sample, the percentage of the injected dose was calculated and normalized according to the tissue weight ( $\%$ ID $/ g_{\text {tissue }}$ ), followed by normalization according to the injected dose and the weight of the animal $\left[\left(\% \mathrm{ID} / \mathrm{g}_{\text {tissue }}\right) /\left(100 \% / \mathrm{g}_{\text {animal }}\right)\right]$ to calculate the standardized uptake value (SUV) for each tissue.

\subsubsection{Metabolism studies}

Mouse blood samples were taken at 30 minutes after intravenous injection of $\sim 40 \mathrm{MBq}$ of $\left[{ }^{18} \mathrm{~F}\right] 7(\mathrm{n}=2)$. Plasma was obtained by centrifugation of blood at $12,000 \mathrm{rpm}$ at room temperature for 1 minute.

MLC: For preparation of the MLC injection samples, mouse plasma ( $20-50 \mu \mathrm{L}, 30$ minutes p.i.) was dissolved in $100-300 \mu \mathrm{L}$ of $100 \mathrm{mM}$ aqueous sodium dodecyl sulfate (SDS). Homogenized brain material ( $200 \mu \mathrm{L}, 30$ minutes p.i.) was dissolved in $400 \mu \mathrm{L}$ of $200 \mathrm{mM}$ aqueous SDS, stirred at $75^{\circ} \mathrm{C}$ for 5 minutes, diluted with more $200 \mu \mathrm{L}$ of $200 \mathrm{mM}$ aqueous SDS and injected into the MLC system after cooling to room temperature. To proof the integrity of the radioligand under these conditions, $50 \mathrm{kBq}$ of the radioligand were stirred at $75{ }^{\circ} \mathrm{C}$ for 5 minutes in $500 \mu \mathrm{L}$ of $200 \mathrm{mM}$ aqueous SDS and analysed via MLC. The MLC system was built up of a JASCO PU-980 pump, an AS-2055Plus auto injector with a $2000 \mu \mathrm{L}$ sample loop, and a UV-1575 detector coupled with a gamma radioactivity HPLC detector (Gabi Star, raytest Isotopenmessgeräte $\mathrm{GmbH}$ ). Data analysis was performed with the Galaxie chromatography software (Agilent Technologies). A Reprosil-Pur C18-AQ column ( $250 \times 4.6 \mathrm{~mm}$, particle size: $10 \mu \mathrm{m}$ ) coupled with a pre-column of $10 \mathrm{~mm}$ length was used. Separations were performed by using an eluent mixture of THF/100 mM aqueous SDS/10 $\mathrm{mM} \mathrm{Na}_{2} \mathrm{HPO}_{4}$ aq. in gradient mode ( $0-10 \mathrm{~min}$ at $5 \% \mathrm{THF}, 10-25 \mathrm{~min}$ up to $50 \% \mathrm{THF}, 25-30 \mathrm{~min}$ at 50\% THF, $30-31 \mathrm{~min}$ up to $5 \%$ THF; $31-40 \mathrm{~min}$ at 5\% THF/100 mM SDS aq., $10 \mathrm{mM} \mathrm{Na} \mathrm{HPO}_{4}$ aq.) at a flow rate of 1.0 $\mathrm{mL} / \mathrm{min}$.

RP-HPLC: For protein precipitation and extraction an ice-cold mixture of acetone/water (9/1; $\mathrm{v} / \mathrm{v}$ ) was used in a ratio of $4: 1$ of solvent to plasma or brain homogenate, respectively. The samples were vortexed for 2 minutes, equilibrated on ice for 10 minutes, and centrifuged for 5 minutes at 10,000 rpm. After separating the supernatant, the precipitates were washed with $200 \mu \mathrm{L}$ of the solvent mixture and subjected to the same procedure. The combined supernatants (total volume between $1.0-1.5 \mathrm{~mL}$ ) were concentrated at $65{ }^{\circ} \mathrm{C}$ under nitrogen flow to a final volume of approximately $100 \mu \mathrm{L}$ and analyzed by analytical radio-HPLC. To determine the percentage of radioactivity in the supernatants compared to total activity, aliquots of each step as well as the precipitates were quantified by $\gamma$ counting (PerkinElmer Wallac Wizard 1480 Gamma Counter, manufactured by WALLAC, Turku, Finland). With this procedure, recoveries of about $97 \%$ for the plasma and brain homogenates could be obtained. 
To analyse the worked-up samples, the same HPLC method was used as described in the radiochemistry part.

\section{Acknowledgements}

The authors would like to thank the China Scholarship Council for supporting the PhD thesis of Dr. Jianrong Liu. Campus France together with the German Academic Exchange Service DAAD kindly supported this project with a travel grant (PHC PROCOPE Project ID: 57129895). The Region Auvergne-Rhone-Alpes supported the contibution of Dr. Jianrong Liu on an international conference. We also thank Dr. K. Franke, Dr. A. Mansel and Dr. S. Fischer for providing $\left[{ }^{18} \mathrm{~F}\right]$ fluoride.

\section{References}

[1] C.A. Peixoto, A.K. Nunes, A. Garcia-Osta, Phosphodiesterase-5 inhibitors: action on the signaling pathways of neuroinflammation, neurodegeneration, and cognition, Mediators Inflamm., 2015 (2015) 940207.

[2] T. Peng, J. Gong, Y. Jin, Y. Zhou, R. Tong, X. Wei, L. Bai, J. Shi, Inhibitors of phosphodiesterase as cancer therapeutics, Eur. J. Med. Chem., 150 (2018) 742-756.

[3] M. Kumazoe, K. Sugihara, S. Tsukamoto, Y. Huang, Y. Tsurudome, T. Suzuki, Y. Suemasu, N. Ueda, S. Yamashita, Y. Kim, K. Yamada, H. Tachibana, 67-kDa laminin receptor increases cGMP to induce cancer-selective apoptosis, J. Clin. Invest., 123 (2013) 787-799.

[4] A. Das, D. Durrant, F.N. Salloum, L. Xi, R.C. Kukreja, PDE5 inhibitors as therapeutics for heart disease, diabetes and cancer, Pharmacol. Ther., 147 (2015) 12-21.

[5] C.M. Whitehead, K.A. Earle, J. Fetter, S. Xu, T. Hartman, D.C. Chan, T.L. Zhao, G. Piazza, A.J. Klein-Szanto, R. Pamukcu, H. Alila, P.A. Bunn, Jr., W.J. Thompson, Exisulind-induced apoptosis in a non-small cell lung cancer orthotopic lung tumor model augments docetaxel treatment and contributes to increased survival, Mol. Cancer Ther., 2 (2003) 479-488.

[6] G.A. Piazza, W.J. Thompson, R. Pamukcu, H.W. Alila, C.M. Whitehead, L. Liu, J.R. Fetter, W.E. Gresh, Jr., A.J. Klein-Szanto, D.R. Farnell, I. Eto, C.J. Grubbs, Exisulind, a novel proapoptotic drug, inhibits rat urinary bladder tumorigenesis, Cancer Res., 61 (2001) 39613968.

[7] F. Karami-Tehrani, M. Moeinifard, M. Aghaei, M. Atri, Evaluation of PDE5 and PDE9 expression in benign and malignant breast tumors, Arch. Med. Res., 43 (2012) 470-475.

[8] A.K. Joe, H. Liu, D. Xiao, J.W. Soh, J.T. Pinto, D.G. Beer, G.A. Piazza, W.J. Thompson, I.B. Weinstein, Exisulind and CP248 induce growth inhibition and apoptosis in human esophageal adenocarcinoma and squamous carcinoma cells, J. Exp. Ther. Oncol., 3 (2003) 8394.

[9] M. Sponziello, A. Verrienti, F. Rosignolo, R.F. De Rose, V. Pecce, V. Maggisano, C. Durante, S. Bulotta, G. Damante, L. Giacomelli, C.R. Di Gioia, S. Filetti, D. Russo, M. Celano, PDE5 expression in human thyroid tumors and effects of PDE5 inhibitors on growth and migration of cancer cells, Endocrine, 50 (2015) 434-441.

[10] B. Zhu, L. Vemavarapu, W.J. Thompson, S.J. Strada, Suppression of cyclic GMP-specific phosphodiesterase 5 promotes apoptosis and inhibits growth in HT29 cells, J. Cell Biochem., 94 (2005) 336-350.

[11] W.J. Thompson, G.A. Piazza, H. Li, L. Liu, J. Fetter, B. Zhu, G. Sperl, D. Ahnen, R. Pamukcu, Exisulind induction of apoptosis involves guanosine 3',5'-cyclic monophosphate 
phosphodiesterase inhibition, protein kinase $\mathrm{G}$ activation, and attenuated beta-catenin, Cancer Res., 60 (2000) 3338-3342.

[12] T.C. Peak, A. Richman, S. Gur, F.A. Yafi, W.J. Hellstrom, The Role of PDE5 Inhibitors and the NO/cGMP Pathway in Cancer, Sex. Med. Rev., 4 (2016) 74-84.

[13] J.L. Roberts, L. Booth, A. Conley, N. Cruickshanks, M. Malkin, R.C. Kukreja, S. Grant, A. Poklepovic, P. Dent, PDE5 inhibitors enhance the lethality of standard of care chemotherapy in pediatric CNS tumor cells, Cancer Biol. Ther., 15 (2014) 758-767.

[14] L. Booth, J.L. Roberts, N. Cruickshanks, S. Tavallai, T. Webb, P. Samuel, A. Conley, B. Binion, H.F. Young, A. Poklepovic, S. Spiegel, P. Dent, PDE5 inhibitors enhance celecoxib killing in multiple tumor types, J. Cell. Physiol., 230 (2015) 1115-1127.

[15] L. Booth, J.L. Roberts, A. Poklepovic, P. Dent, PDE5 inhibitors enhance the lethality of [pemetrexed + sorafenib], Oncotarget, 8 (2017) 13464-13475.

[16] P.R. Ding, A.K. Tiwari, S. Ohnuma, J.W. Lee, X. An, C.L. Dai, Q.S. Lu, S. Singh, D.H. Yang, T.T. Talele, S.V. Ambudkar, Z.S. Chen, The phosphodiesterase-5 inhibitor vardenafil is a potent inhibitor of ABCB1/P-glycoprotein transporter, PLoS One, 6 (2011) e19329.

[17] Z. Shi, A.K. Tiwari, S. Shukla, R.W. Robey, S. Singh, I.W. Kim, S.E. Bates, X. Peng, I. Abraham, S.V. Ambudkar, T.T. Talele, L.W. Fu, Z.S. Chen, Sildenafil reverses ABCB1- and ABCG2-mediated chemotherapeutic drug resistance, Cancer Res., 71 (2011) 3029-3041.

[18] G. Ribaudo, M.A. Pagano, S. Bova, G. Zagotto, New Therapeutic Applications of Phosphodiesterase 5 Inhibitors (PDE5-Is), Curr. Med. Chem., 23 (2016) 1239-1249.

[19] D.G. Cooper, I.T. Forbes, V. Garzya, D.J. Johnson, G.I. Stevenson, P.A. Wyman, Preparation of cyclohexylpiperidinylbenzoxazolone derivatives and analogs as M1 receptor modulators., WO 2009037294A1, (2009).

[20] S. Schröder, B. Wenzel, W. Deuther-Conrad, M. Scheunemann, P. Brust, Novel Radioligands for Cyclic Nucleotide Phosphodiesterase Imaging with Positron Emission Tomography: An Update on Developments Since 2012, Molecules, 21 (2016).

[21] S. Jakobsen, G.M. Kodahl, A.K. Olsen, P. Cumming, Synthesis, radiolabeling and in vivo evaluation of $\left[{ }^{11} \mathrm{C}\right] \mathrm{RAL}-01$, a potential phosphodiesterase 5 radioligand, Nucl. Med. Biol., 33 (2006) 593-597.

[22] V. Gomez-Vallejo, A. Ugarte, C. Garcia-Barroso, M. Cuadrado-Tejedor, B. Szczupak, I.G. Dopeso-Reyes, J.L. Lanciego, A. Garcia-Osta, J. Llop, J. Oyarzabal, R. Franco, Pharmacokinetic investigation of sildenafil using positron emission tomography and determination of its effect on cerebrospinal fluid cGMP levels, J. Neurochem., 136 (2016) 403415.

[23] R. Chekol, O. Gheysens, J. Cleynhens, P. Pokreisz, G. Vanhoof, M. Ahamed, S. Janssens, A. Verbruggen, G. Bormans, Evaluation of PET radioligands for in vivo visualization of phosphodiesterase 5 (PDE5), Nucl. Med. Biol., 41 (2014) 155-162.

[24] R. Chekol, O. Gheysens, M. Ahamed, J. Cleynhens, P. Pokreisz, G. Vanhoof, S. Janssens, A. Verbruggen, G. Bormans, Carbon-11 and Fluorine-18 Radiolabeled Pyridopyrazinone Derivatives for Positron Emission Tomography (PET) Imaging of Phosphodiesterase-5 (PDE5), J. Med. Chem., 60 (2017) 486-496.

[25] Y. Bi, P. Stoy, L. Adam, B. He, J. Krupinski, D. Normandin, R. Pongrac, L. Seliger, A. Watson, J.E. Macor, Quinolines as extremely potent and selective PDE5 inhibitors as potential agents for treatment of erectile dysfunction, Bioorg. Med. Chem. Lett., 14 (2004) 1577-1580. [26] J. Fiorito, F. Saeed, H. Zhang, A. Staniszewski, Y. Feng, Y.I. Francis, S. Rao, D.M. Thakkar, S.X. Deng, D.W. Landry, O. Arancio, Synthesis of quinoline derivatives: discovery of a potent and selective phosphodiesterase 5 inhibitor for the treatment of Alzheimer's disease, Eur. J. Med. Chem., 60 (2013) 285-294.

[27] S.J. Lee, Y. Konishi, D.T. Yu, T.A. Miskowski, C.M. Riviello, O.T. Macina, M.R. Frierson, K. Kondo, M. Sugitani, J.C. Sircar, K.M. Blazejewski, Discovery of potent cyclic GMP phosphodiesterase inhibitors. 2-Pyridyl- and 2-imidazolylquinazolines possessing cyclic 
GMP phosphodiesterase and thromboxane synthesis inhibitory activities, J. Med. Chem., 38 (1995) 3547-3557.

[28] J. Liu, A. Maisonial-Besset, B. Wenzel, D. Canitrot, A. Baufond, J.M. Chezal, P. Brust, E. Moreau, Synthesis and in vitro evaluation of new fluorinated quinoline derivatives with high affinity for PDE5: Towards the development of new PET neuroimaging probes, Eur. J. Med. Chem., 136 (2017) 548-560.

[29] J. Liu, B. Wenzel, S. Dukic-Stefanovic, R. Teodoro, F.A. Ludwig, W. Deuther-Conrad, S. Schröder, J.M. Chezal, E. Moreau, P. Brust, A. Maisonial-Besset, Development of a New Radiofluorinated Quinoline Analog for PET Imaging of Phosphodiesterase 5 (PDE5) in Brain, Pharmaceuticals 9(2016).

[30] F. Dolle, Fluorine-18-labelled fluoropyridines: advances in radiopharmaceutical design, Curr. Pharm. Des., 11 (2005) 3221-3235.

[31] SB Drug Discovery; www.sbdrugdiscovery.com.

[32] L. Zhang, A. Villalobos, E.M. Beck, T. Bocan, T.A. Chappie, L. Chen, S. Grimwood, S.D. Heck, C.J. Helal, X. Hou, J.M. Humphrey, J. Lu, M.B. Skaddan, T.J. McCarthy, P.R. Verhoest, T.T. Wager, K. Zasadny, Design and selection parameters to accelerate the discovery of novel central nervous system positron emission tomography (PET) ligands and their application in the development of a novel phosphodiesterase 2A PET ligand, J. Med. Chem., 56 (2013) 45684579.

[33] H.H. Coenen, Fluorine-18 labeling methods: Features and possibilities of basic reactions, Ernst Schering Res. Found. Workshop, (2007) 15-50.

[34] O. Jacobson, D.O. Kiesewetter, X. Chen, Fluorine-18 radiochemistry, labeling strategies and synthetic routes, Bioconjug. Chem., 26 (2015) 1-18.

[35] D.S. Noyce, J.A. Virgilio, Investigation of Rate of Hydrolysis of 1-Phenylethyl Phenylphosphinate as a Function of pH, J. Org. Chem., 37 (1972) 1052-1053.

[36] M.M. Claffey, C.J. Helal, P.R. Verhoest, Z. Kang, K.S. Fors, S. Jung, J. Zhong, M.W. Bundesmann, X. Hou, S. Lui, R.J. Kleiman, M. Vanase-Frawley, A.W. Schmidt, F. Menniti, C.J. Schmidt, W.E. Hoffman, M. Hajos, L. McDowell, R.E. O'Connor, M. MacdougallMurphy, K.R. Fonseca, S.L. Becker, F.R. Nelson, S. Liras, Application of structure-based drug design and parallel chemistry to identify selective, brain penetrant, in vivo active phosphodiesterase 9A inhibitors, J. Med. Chem., 55 (2012) 9055-9068.

[37] Z. Li, P.S. Conti, Radiopharmaceutical chemistry for positron emission tomography, Adv. Drug Deliv. Rev., 62 (2010) 1031-1051.

[38] D.W. Kim, H.J. Jeong, S.T. Lim, M.H. Sohn, J.A. Katzenellenbogen, D.Y. Chi, Facile nucleophilic fluorination reactions using tert-alcohols as a reaction medium: significantly enhanced reactivity of alkali metal fluorides and improved selectivity, J. Org. Chem., 73 (2008) 957-962.

[39] D.W. Kim, D.S. Ahn, Y.H. Oh, S. Lee, H.S. Kil, S.J. Oh, S.J. Lee, J.S. Kim, J.S. Ryu, D.H. Moon, D.Y. Chi, A new class of $S_{N} 2$ reactions catalyzed by protic solvents: Facile fluorination for isotopic labeling of diagnostic molecules, J. Am. Chem. Soc., 128 (2006) 16394-16397.

[40] P. Marchand, A. Ouadi, M. Pellicioli, J. Schuler, P. Laquerriere, F. Boisson, D. Brasse, Automated and efficient radiosynthesis of $\left[{ }^{18} \mathrm{~F}\right] \mathrm{FLT}$ using a low amount of precursor, Nucl. Med. Biol., 43 (2016) 520-527.

[41] H.S. Kil, H.Y. Cho, S.J. Lee, S.J. Oh, D.Y. Chi, Alternative synthesis for the preparation of 16alpha- $\left[{ }^{18}\right.$ F]fluoroestradiol, J. Labelled Comp. Radiopharm., 56 (2013) 619-626.

[42] M. Suehiro, S. Vallabhajosula, S.J. Goldsmith, D.J. Ballon, Investigation of the role of the base in the synthesis of $\left[{ }^{18}\right.$ F]FLT, Appl. Radiat. Isot., 65 (2007) 1350-1358.

[43] J.H. Chun, S. Telu, S.Y. Lu, V.W. Pike, Radiofluorination of diaryliodonium tosylates under aqueous-organic and cryptand-free conditions, Org. Biomol. Chem., 11 (2013) 50945099. 
[44] L. Brichard, F.I. Aigbirhio, An Efficient Method for Enhancing the Reactivity and Flexibility of $\left[{ }^{18} \mathrm{~F}\right]$ Fluoride Towards Nucleophilic Substitution Using Tetraethylammonium Bicarbonate, Eur. J. Org. Chem., (2014) 6145-6149.

[45] J.H. Chun, S.Y. Lu, Y.S. Lee, V.W. Pike, Fast and High-Yield Microreactor Syntheses of ortho-Substituted $\left[{ }^{18} \mathrm{~F}\right]$ Fluoroarenes from Reactions of $\left[{ }^{18} \mathrm{~F}\right]$ Fluoride Ion with Diaryliodonium Salts, J. Org. Chem., 75 (2010) 3332-3338.

[46] D. Landini, A. Maia, A. Rampoldi, Dramatic Effect of the Specific Solvation on the Reactivity of Quaternary Ammonium Fluorides and Poly(Hydrogen Fluorides), $(\mathrm{HF})_{\mathrm{n}} \mathrm{F}^{-}$, in Media of Low Polarity, J. Org. Chem., 54 (1989) 328-332.

[47] A.F. Teich, M. Sakurai, M. Patel, C. Holman, F. Saeed, J. Fiorito, O. Arancio, PDE5 Exists in Human Neurons and is a Viable Therapeutic Target for Neurologic Disease, J. Alzheimers Dis., 52 (2016) 295-302.

[48] P. Cumming, A business of some heat: molecular imaging of phosphodiesterase 5, J. Neurochem., 136 (2016) 220-221.

[49] D. Giordano, M.E. De Stefano, G. Citro, A. Modica, M. Giorgi, Expression of cGMPbinding cGMP-specific phosphodiesterase (PDE5) in mouse tissues and cell lines using an antibody against the enzyme amino-terminal domain, Biochim. Biophys. Acta, 1539 (2001) 1627. 